\title{
SEISMIC PERFORMANCE INVESTIGATION OF STEEL MOMENT CONNECTION WITH REDUCED BEAM SECTION BY USING FINITE ELEMENT ANALYSIS METHOD
}

\author{
A Thesis submitted to the faculty of \\ San Francisco State University \\ In partial fulfillment of \\ the requirements for \\ the Degree \\ Master of Science \\ In \\ Engineering: Sturctural/Earthquake Engineering \\ by \\ Yuanhong Chen \\ San Francisco, California
}

May 2020 


\section{Copyright by}

Yuanhong Chen

2020 


\section{CERTIFICATION OF APPROVAL}

I certify that I have read SEISMIC PERFORMANCE INVESTIGATION OF STEEL MOMENT CONNECTION WITH REDUCED BEAM SECTION BY USING FINITE ELEMENT ANALYSIS METHOD by Yuanhong Chen, and that in my opinion this work meets the criteria for approving a thesis submitted in partial fulfillment of the requirement for the degree Master of Science in Engineering: Structural/Earthquake Engineering at San Francisco State University.

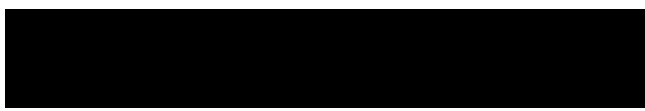

Cheng Chen, Ph.D.

Associate Professor of Engineering

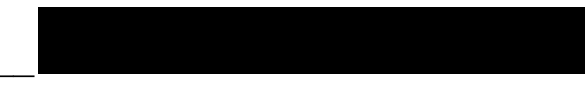

Wenshen Pong, Ph.D. Professor of Engineering 


\title{
SEISMIC PERFORMANCE INVESTIGATION OF STEEL MOMENT CONNECTION WITH REDUCED BEAM SECTION BY USING FINITE ELEMENT ANALYSIS METHOD
}

\author{
Yuanhong Chen \\ San Francisco, California
}

2020

Finite element analysis method has been widely using for seismic performance investigation of different type of structural materials including steel, concrete, composite etc. A model of moment resisting frame with the reduced beam section (RBS) was created by using finite element software Strand 7 and the push over nonlinear static analysis was performed to investigate the seismic behavior of the frame. Analytical result showed expected well nonlinear characteristics of the hysteresis of the steel moment frame under cyclic loading. Furthermore, the moment frame connection exhibit less strength deterioration compared to the moment frame without RBS. To exam the nonlinear seismic performance of the RBS under earthquake ground motion, time history analysis was conducted by using the nonlinear transient solver from Strand7. Moment vs. Rotation result plots were generated to reveal the response of the frame under the simulated earthquake ground motion. On the hand, in order to conduct an inquiry into the effect of secondary moment to the frame with RBS, estimated axial loads were applied to the columns of the connection for the push over nonlinear static analysis. Analytical 
results show that the peak strength of the RBS moment connection doesn't have significant decreases due to the P-Delta effect within axial load which is equivalent to $10 \%$ of column compression capacity, Furthermore, foreseen of degree of column strength deterioration can be efficiently predicted with FEA in Strand7.

I certify that the Abstract is a correct representation of the content of this thesis.
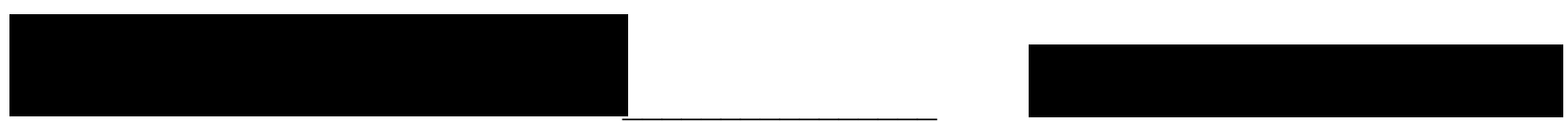

Chair. Thesis Committee

Date 


\section{PREFACE AND/OR ACKNOWLEDGEMENTS}

First, I would like to thank my family for the support they have given me throughout structural engineering graduate school endeavors. I would like to thank my father, Yongshu Chen, who helped me with school tuition and provide financial assistance for my education. Furthermore, he has always considered family first, which I always look

up to. His encouragement in my higher education was unwavering. My mother, Shaoling Chen, who has good discipline and always support me with her love. To my brother Jinrong Chen and his family, thanks for sharing their life with me. To my partner Tim Chan who has guided and helped me through much of my life. To my two daughters who has given me joy and motivation while I was working as an EIT and pursuing higher education. A special thanks to my graduate advisor Dr. Cheng Chen, as he took the time to encourage, motivate, and guide me in my studies and research project. His comments and guidance are extremely valuable for my life. Dr. Chen's dedication to teaching is appreciated and enduring. Thank you to Strand7 software support team's effort for helping me with research project and their excellent communication performance with research assistance. Thank you to all the San Francisco State University professors and the San Francisco State University Engineering program for contributing to my education and help establish my career.

Lastly, I would like to thank my classmates and friends for their inspiration of new concept and ideas. Their competitive nature motivates me to improve and upgrade myself all the time. 


\section{TABLE OF CONTENTS}

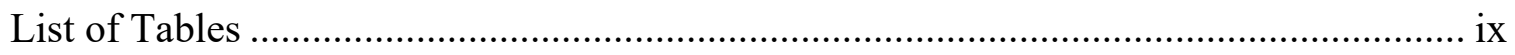

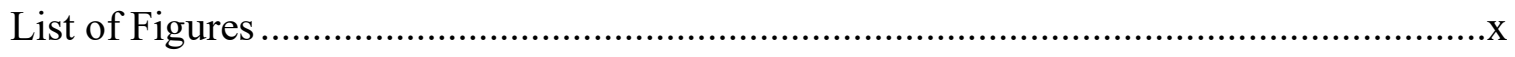

Chapter 1 Introduction ............................................................................................

1.1 Background on Finite Element Analysis ........................................................

1.2 Reduced Beam Section Connection....................................................................2

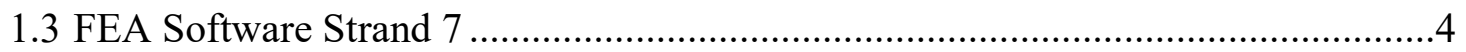

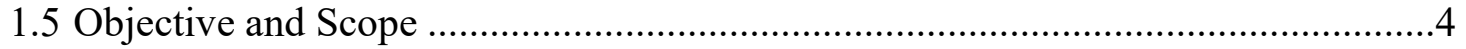

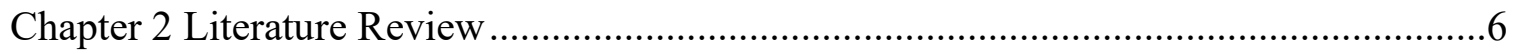

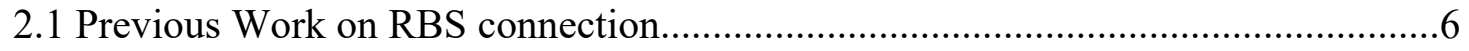

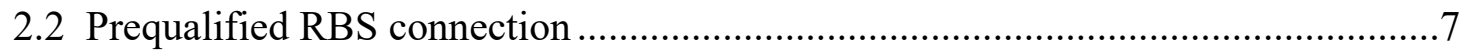

2.2-1 Pre-Northridge Connection and Post-Northridge Design .................................

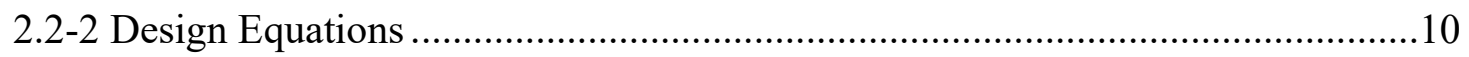

2.2.3 Welding and Detailing of RBS for Special Moment Frame System ..................11

Chapter 3 Finite Element Modeling and Analysis of RBS connection ..........................19

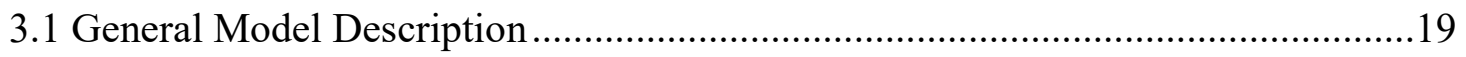

3.2 Finite Element Models of RBS with Strand7 .................................................20

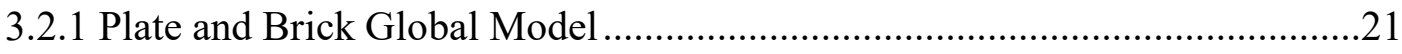

3.2.2 Models with Plate Element .................................................................22

3.2.2 Modeling with Brick Element .................................................................23

3.2.3 FEA Models' Boundary Condition and Loading Protocol.............................24

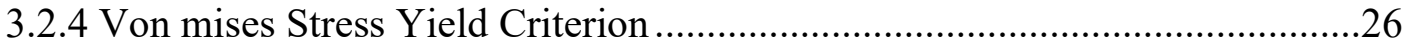




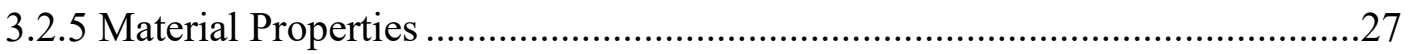

3.2.6 Geometric Nonlinearity In Finite Element Analysis........................................29

3.3 Finite Element Analysis Results ........................................................................

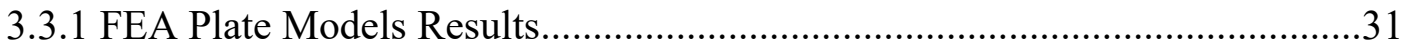

3.3.2 DB1 Constant Cut RBS Connection Model FEA Results.................................32

3.3.3 DB1 Beam Lateral Torsional Deformation Studies...........................34

3.3.4 DB2 Radius Cut RBS Connection Model FEA Results.....................................36

3.3.5 Beam moment analysis of RBS connection ....................................................37

Chapter 4 FEA of Steel Moment Resisting Frame (SMRF) with RBS connection............64

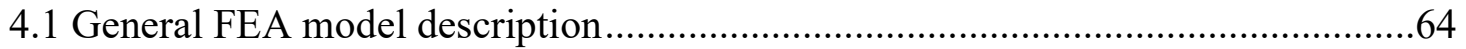

4.2 Translational mass determination ...................................................................65

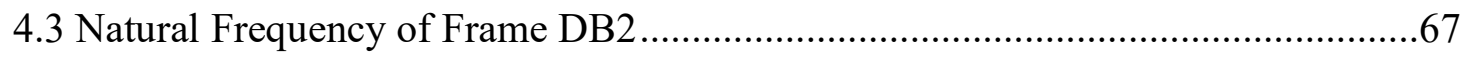

4.4 Nonlinear Time history Analysis of SMRF with Strand7 Transient Dynamic

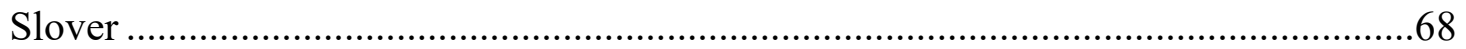

4.5 Lateral Bracing Force Estimation …………………….........................................

4.6 Nonlinear Static Pushover Analysis of RBS Moment Frame Model.......................72

4.7 Incremental Dynamic Analysis ...........................................................................74

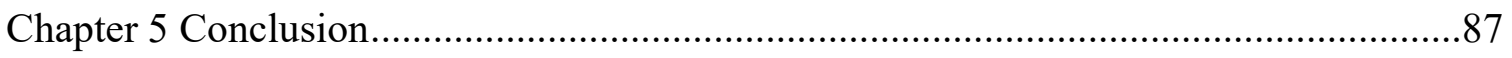

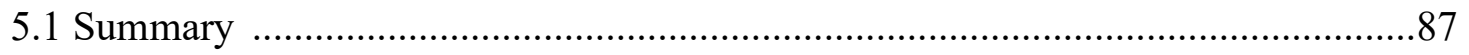

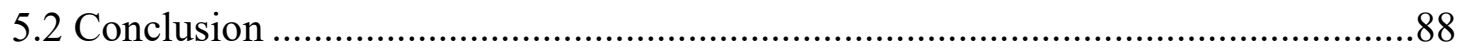

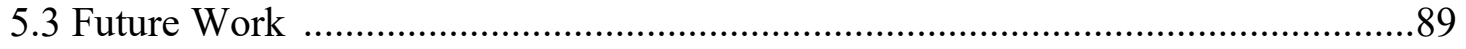

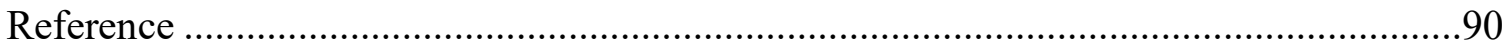

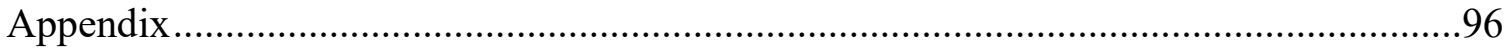




\section{List of Tables}

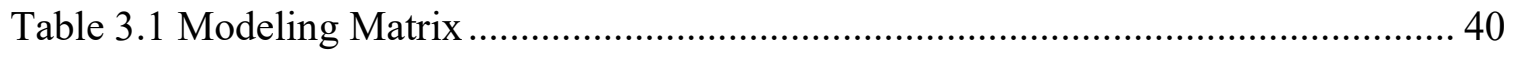

Table 3.2 Loading History for Specimens (Modified).............................................. 40

Table 3.3 Connection Sections Material Properties From Coupon Tensil Test

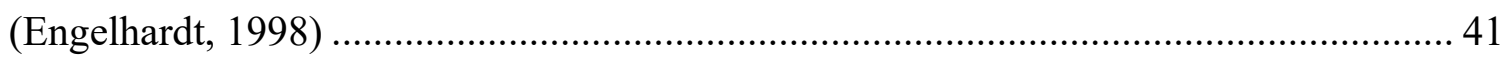

Table 3.4 Plate Model Information of DB1 and DB2 ............................................. 41

Table 3.5 Beam Moments of DB1 and DB2 (FEA Plate Model) .................................. 41

Table 3.6 Analysis matrix to evaluate effect of axial load on cyclic global performance of

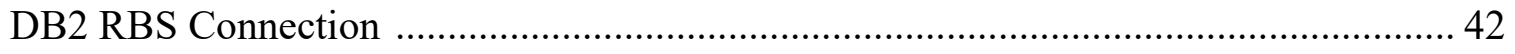

Table 4.1 FEA Natural Frequency Analysis Results for Frame with Full Beam Section 74

Table 4.2 FEA Natural Frequency Analysis Results for Frame with RBS.................... 74

Table 4.3 Lateral Forces of Beam Flanges for Frame with RBS ................................ 74

Table A.1Procedure to Calculate Node Mass for RBS MRF ...................................... 88 


\section{List of Figures}

Figure 2.1 Pre-Northridge Connection Detail (Civjan et al. 2000)............................... 15

Figure 2.2 Post-Northridge Connection Detail (Stojadinovic et al.,2000) ..................... 16

Figure 2.3 Post-Northridge Connection with......................................................... 16

Figure 2.4 Cover Plate Connection (Deylami \& Gholipour, 2011) .............................. 17

Figure 2.5 Reduced Beam Section Connection (AISC 358,2015) ............................... 17

Figure 2.6 Detailing of RBS Design (2012 IBC, volume 4) ....................................... 18

Figure 3.1 DB1 Dog Bone Connection Detail ( Engelhardt et al. 1998) ........................ 43

Figure 3.2 DB2 Dog Bone Connection Detail ( Engelhardt et al. 1998) ......................... 43

Figure 3.3 Nonlinear Static Solver Convergency Graph (Strand7, 2015) ..................... 44

Figure 3.4 RBS Test Setup ( Engelhardt et. al, 1998)............................................... 44

Figure 3.5 Global model span length and boundary condition for FEA ....................... 45

Figure 3.6 3D view of DB2 Plate FEA Model (Strand7, 2016) ................................. 45

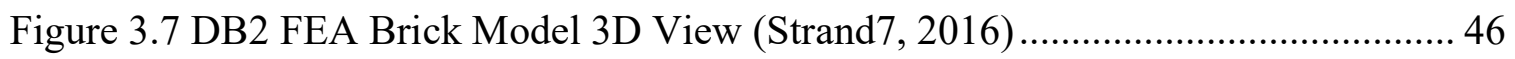

Figure 3.8 Plane View of DB1Brick Model- Constant Cut RBS .................................. 47

Figure 3.9 Plan View of DB2Brick Model-Radius Cut RBS ..................................... 47

Figure 3.10 Plate Elements for RBS FEA Modeling................................................ 48

Figure 3.11 FEA Model's Boundary Conditions and Applied Displacement Load ......... 48

Figure 3.12 Deformed Shape of the RBS Connection During Cyclic Testing ................ 49

Figure 3.13 ATC-24 Loading Protocol with Displacement Loading ............................ 49

Figure 3.14 Modified Stress vs Strain Curve For Beam Flange ................................... 50

Figure 3.15 Modified Stress vs Strain Curve For Beam Web ..................................... 50

Figure 3.16 Modified Stress vs Strain For Column Flange ....................................... 51

Figure 3.17 Modified Stress vs Strain Curve For Column Web................................... 51

Figure 3.18 Bill of Material of Plate DB2 Model .................................................... 52 
Figure 3.19 Discrepancy Between Analysis Results and Input Stress-Strain Curve For Typical Mil Steel Material ( Strand7 Webnote) .......................................................... 52

Figure 3.20 DB1 Plate Model Load vs Deflection Plot ................................................. 53

Figure 3.21 DB1 Brick Model Load vs Deflection Plot ................................................ 53

Figure 3.22 Von Mises Stress of DB1 Model at 0.05 radian Rotation ( $0 \%$ displacement

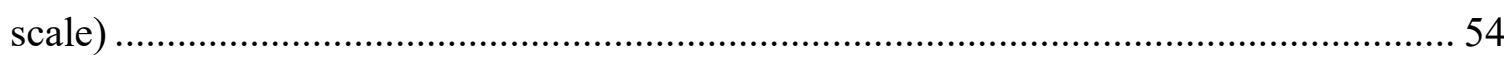

Figure 3.23 Deformed Shape of DB1 Plate Model at 0.05 Radian Rotation................... 54

Figure 3.24 Deformed Shape of DB1 Brick Model at 0.05 Radian Rotation................... 55

Figure 3.25 Beam Lateral Out of Plane Displacement of DB1 Plate Model.................. 55

Figure 3.26 Beam Lateral Out of Plane Displacement of DB1 Brick Model .................. 56

Figure 3.27 Comparison of connection with and without RBS (DB1)......................... 56

Figure 3.28 DB1 Beam Flange Out of Plane Displacement ........................................ 57

Figure 3.29 DB1 Beam Web Out of Plane Displacement ......................................... 58

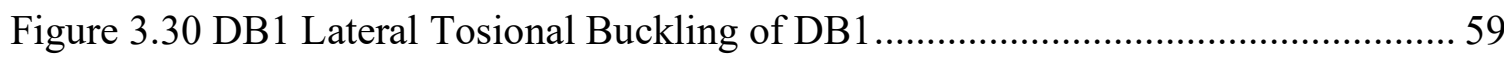

Figure 3.31 DB1 Plate Model Moment vs Rotation Plot............................................ 59

Figure 3.32 DB1 Brick Model Moment vs. Rotation Plot............................................ 60

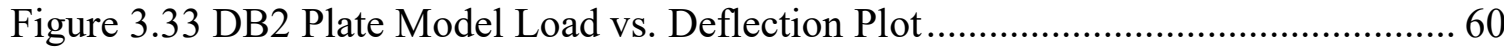

Figure 3.34 DB2 Brick Model Load vs. Deflection Plot ............................................... 61

Figure 3.35 DB2 Plate Model Moment vs Rotation Plot............................................ 61

Figure 3.36 DB2 Brick Model Moment vs Rotation Plot........................................... 62

Figure 3.37 Comparison of cyclic behavior of cases with different axial load .............. 62

Figure 3.38 Yield Index Contour for DB2 Models with various axial load effects......... 64

Figure 4.1 FEA Model of Steel Moment Frame with RBS ........................................ 75

Figure 4.2 Failure modes of the FEA Frame Model ................................................... 75

Figure 4.3 Selected Acceleration vs Time Ground Motion for Moment Frame Models.. 76 Figure 4.4 RBS Moment Frame Roof Displacement Time History 77 
Figure 4.5 RBS Moment Frame Roof Displacement Time History .............................. 77

Figure 4.6 Full Beam Section Moment Frame Roof Displacement Time History ........... 78

Figure 4.7 DB2 Moment Frame Beam Flange Lateral Bracing.................................. 78

Figure 4.8 DB2 Moment Frame Beam Flange Lateral Bracing Force History ............... 79

Figure 4.9 DB2 Beam Flange Lateral Force History for Case 1 and Case 3 ................. 80

Figure 4.10 DB2 Beam Flange Lateral Force History for Case 2 and Case 4 ............... 81

Figure 4.11 Deformed shape of RBS Moment Frame (NSA) .................................... 82

Figure 4.12 Deformed Shape of RBS Moment Frame (THA) .................................... 82

Figure 4.13 Base Shear vs Displacement ( Nonlinear Pushover Analysis) ................... 83

Figure 4.14 RBS Moment Frame Base Shear vs Displacement Time History ................ 83

Figure 4.15 Full Beam Section Moment Frame Base Shear vs Displacement ................ 84

Figure 4.16 Structural Response of DB2 Moment Frame: Peak Ground ....................... 84

Figure 4.17 Out of Plane Lateral Displacement of Beam Web (2.1x scaled)................. 85

Figure 4.18 Out of Plane Lateral Displacement of Beam Web (2.2x scaled)................. 85 


\section{Chapter 1}

\section{Introduction}

\subsection{Background on Finite Element Analysis}

Steel Connection design had been changed after the 1994 catastrophic Northridge earthquake. Design codes had been questioned due the failure of steel moment resisting frame during the quake (Ajay and Gaurang, 2013). The connections damage of the frames is the main concern that has been addressed in the current design code (Ferraioli et al., 2014). And post Northridge connections are the acceptable design which can prevent brittle failure of the beam column connections as stated in recent seismic design guidelines. Full-scale experimental studies of steel moment frame (SMF) have been done by researchers Elkady and Lignos (2017) to show the performance of SMF under seismic loading and the results of the simulation can be used to validate the design code requirements and standards. However, Full-scale experimental studies are not cost effective due to their expensive set up in the laboratories and the required large spatial room to be accurately record testing data. On the other hand, analytical studies are important parts of research that to validate experimental results to be feasible and possibly improve connection design with good understanding of the nonlinear behavior of steel moment connections. 
Finite element Modeling (FEM) is a preferable choice for analytical model of the testing (Ebrahimi et al., 2018). A few FEM software have been used in studies of structural SMF under cyclic seismic loading and the results are verified with experimental data (Moradi and Alam, 2015). But more verifications can be done with new FEM software Strand7 to validate the computational results and to capture the stress formation locations which it was not able to be easily detected in the experimental testing. Nevertheless, the practical and efficient modeling methodology need to be studied and developed systematically in Strand7 to provide good analytical results for verification with the experimental results, furthermore, supply rational calibration of design model (Mashaly et al,2011).

\subsection{Reduced Beam Section Connection}

Reduced Beam Section (RBS) moment connection is one of the well-known connections that is used for steel moment resisting frame after the 1994 Northridge Earthquake due to its good seismic performance compared to other traditional moment connection design developed before the quake. This type of connection enhances the moment frame's capability to develop ductile behavior without fracture near the column face of the connection under seismic loading (Zhang, 2004).

Therefore, it complies the strong column weak beam design concept for steel moment resisting frame (AISC 341,2016). As the name implies, the sum of the column flexure strength must exceed the sum of the beam flexural strength at each joint of the frame (Dardis,2016). In other words, the beams should fail (or form plastic hinges) prior to 
the columns, in order to avoid progressive collapse of a structure due to column failure in the lower levels. Majority of testing with moment resisting frame From SAC Joint Venture (2000) data base are with shallow column (W14) of prequalified connections. Though among the test results, RBS was one of the cost-effective connections with quality seismic performance.

\subsection{FEA Software Strand 7}

Strand 7 is a Finite Element Analysis software which can conduct modeling with varies types of elements including beam, plate, brick, depends on the degree of accuracy for specific models. Each element type has its own characteristics to capture the deformations or displacements of specific type of material modeling. Furthermore, it provides different solver types for nonlinear analysis which is required for comparison and insights with experimental testing results. Due to Strand 7's good modeling graphic interface, it is efficient and flexible to model different type of steel moment connections and generate results in a reasonable solution time requirement. On the other hand, its high quality of post processing plays an important role of extracting result with predictable degree of accuracy (Strand 7 Reference Manual, 2015). 


\subsection{Motivation of Study}

Experimental studies have provided good data and design recommendations for design of RBS moment connection. Though high cost and great effort to conducted full scale experiment make it with limited flexibility to test connection with different section parameters. With finite element modeling and analysis available for understanding the nonlinear behavior of steel moment connections, researchers and engineers can have the adequate data to predict the seismic performance of RBS under earthquake simulations. Furthermore, the analytical modeling can extend to the whole moment frame level with dynamic analysis with selected ground motion simulation in Finite Element Analysis software Strand7. Consequently, valuable prediction can be made to design steel moment frame with RBS to withstand certain magnitude level of earthquake events.

\subsection{Objective and Scope}

The research presented herein explained the modeling the exterior steel beam column connections with RBS by using Strand 7 and finite element analysis was conduct for nonlinear pushover. Moreover, moment resisting frame with RBS was modelled to demonstrate nonlinear dynamic analysis under selected earthquake ground motion simulation. The configuration modeled was a reduced beam section beam known as DB1 and DB2 from reference of test program which was conducted and published by Dr. Michael D. Engelhardt et al. (1998). Finite element study results are then compared to the experimental result to show the degree of accuracy of the analytical modeling. The 
moment resisting frame with RBS modeled was one bay one story steel building model with seismic mass assigned to the column top. The nonlinear response of the structural frame during the 3D dynamic analysis was investigated and compared to the results with a moment frame with full beam section. The moment resisting model is designed based on the properties of DB2 in order to investigate the RBS's performance for the seismic resistance to the frame. The correct boundary condition and material nonlinear properties are defined in respective paper, they are used in Strand 7 to closely model the specimen under the corresponding experimental testing. A modified loading protocol with displacement loading was used to conduct nonlinear push over analysis according to the dimensions of the connection. Moreover, a computer animation of the model undergo cyclic loading and seismic ground motion simulation will be presented as well. 


\section{Chapter 2}

\section{Literature Review}

\subsection{Previous Work on RBS connection}

The need to understand the behavior of the beam to column connection on the moment resisting fame (MRF) has been recognized especially after the 1994 Northridge Earthquake. Researchers Engelhardt (1998) et al. conducted investigation of RBS moment connection from experimental test program for evaluating the design to use in a 25story steel building. The performance of five large specimens with dog-bone type RBS were tested and all of them showed sufficient seismic performance. RBS is a type of steel prequalify connection by cutting part of the beam flange to force the plastic hinge to form in the reduced section, therefore, to avoid failure near the column face and beam web weld connection location (Jones et al, 2002). Prior to the testing focusing about the ductility of the RBS connection, Researcher Jones et al. (2002) also examine how the panel zone strength affect the stability of the connection to develop the hysteretic response at corresponding large seismic story drift level.

Due to deep column's popularity in steel moment frame design for drift control, Chi and Uang (2000) studied the cyclic response of RBS moment connections with deep columns compared to the shallow wide flange column sections such as W14 shapes. The result show that twisting in column was caused by lateral-torsional buckling of the beam. Furthermore, they proposed a design procedure for evaluating the column twisting and 
recommend additional bracing near the RBS region when there is high stress indication happens. Zhang et al. (2006) incorporated Finite Element Modeling analytical studies with experimental testing results on seismic behavior RBS connections with a deep column with wide flange. The performance of the test specimen developed adequate ductility according to the code AISC (2002) seismic design criterial at that time.

More recently, Kulkarni and Vesmawala (2014) conducted experimental and finite element study for exterior moment connections with RBS specimen and WRBS (without RBS) specimen which are designed for Indian Profile. Nevertheless, the specimens' design was based on specification from US AISC (2010) and FEMA (2000) codes. Base on their research, both the experimental and numerical results showed RBS moment connection had no observed column flange or web buckling to indicate good cyclic performance while failure mode was shown in WRBS connection. In addition, there was correlation between the Finite Element Analysis and experiment results that both agree with the increased ductility for RBS connection and inelastic deformation happen in RBS area of the beam, compared to the panel zone and beam flange deformation within WRBS.

\subsection{Prequalified RBS connection}

\subsubsection{Pre-Northridge Connection and Post-Northridge Design}

The prior traditional steel moment frame performance and design Showed weakness dramatically after the 1994 Northridge Earthquake following the 1995 Kobe Earthquake. The weakness of the connection included column fracture occurred in 
buildings at the beam-column joint, and weld metal was found to have inadequate toughness and ductility to handle the cyclical loading associated with earthquakes. Afterwards research and testing of "pre-Northridge" moment frame connections revealed several other potential failure modes with steel moment frames such as weak columnstrong beam configurations, "soft" story behavior excessive building drifts etc. (FORELL/ELSESSER Engineers, 2019).

The Pre-Northridge connection which was widely used in structural steel construction industry before the Northridge Earthquake is shown in Figure 2.1. This type of connection includes the beam flange welded to column with complete joint penetration (CJP) welds. Yet as it is developed in late 1960's and early 1970's, the beam web was bolted to the shear tab and the weld metal was not specified for toughness requirements (Stojadinovic et al. 2000). There were serious damage and failure of this type of connection during Northridge Earthquake (FEMA-267, 1995), and brittle failure modes were observed in experimental testing which was initiated by SAC Steel Project Phase 1 (Malley, 1998) to investigate the cause of the damage of the connection. In most testing cases, the crack of CJP weld caused the fracture of the beam column connection, as a result, before any expected yielding of the beam or column panel zone, brittle fractures developed, as indicated by the small plastic rotation capacity of 0.009 radian (Fry, 1998). After various type of testing had done by SAC Joint Venture to look for means to mitigate brittle fracture of the weld beam column connection, the Post-Northridge connection design detail as shown in Figure 2.2, was implemented to ensure the plastic hinge forms in the beam instead of the connection welds (Stojadinovic et al., 2000). Researchers Civjan et al. (2000) stated that these improved post Northridge connections 
utilized higher toughness electrodes for weld improvements as well as design modification for better seismic performance.

Yet researcher Stojadinovic et al. (2000) pointed out that the rotation capacity of the tested specimen with this type of connection along was found to be not sufficient to withstand high seismic level earthquake; therefore, besides the welding fracture consideration, flange overload issue accompanied within the testing also need to be addressed. Reduced Beam section (RBS) is one of the prequalified Post-Northridge connections that ensure yielding and hinge formation occurs mainly within the reduced section of the beam to achieve the strong column weak beam design goal for special moment frame connections.

Another type of improved moment connection called Cover Plate connection, was also widely used in practice after the Northridge Earthquake, which is shown in Figure 2.3. It was the first improved connection that was testing by using large scale experimental specimen and exhibited good increase of ductility performance compared to the Pre-Northridge connection that showed in Figure 2.3 and 2.4. Nevertheless, difficulties to construct the welding on cover plate connection and it had limited area of inspection for welds, made cover plate connection not a favorable connection to use in steel construction. Hence it was not preferred to use in reinforcement around connections or for building retrofits for better seismic performance, as explained by Engelhardt et al. (1998). Contrarily, RBS connection was a good alternative for steel moment connection since it exhibited improved moment ductility during experimental testing (Civjin et al, 2000). Due to the trimmed portion of beam in the RBS, yielding occurs away from the column face groove welds, hence welds are protected from failure under certain 
earthquake effect. Overall, the connection was stronger than the beam by using RBS as well as it was more economical than the cover plate connection, since it doesn't require to construct big size of the welds for plates (Engelhardt et al.,1998).

\subsubsection{Design Equations}

For RBS design consideration, the cut of the reduced beam section needs to be determined based on iteration of an optimized design for good seismic performance from the connection (Zhang, 2004). The early design was developed by Dr. Engelhardt (1998) and later appeared in the 2016 version of the AISC standard, Prequalified Connections for Special and Intermediate Steel Moment Frames for Seismic Applications (ANSI/AISC 358-16). Below is the design procedure according to the standard of steps of comparing the flexural strength of the beam with the moment developed

1. According to document, the dimensions for the radius cut for the reduced beam section as shown in Figure 2.5 should follow the following limitation:
$0.50 b_{b r} \leq a \leq 0.75 b_{b r}$
AISC 358, Eq 5.8-1
$0.65 d \leq b \leq 0.85 d$
AISC 358 , Eq 5.8-2
$0.10 b_{b r} \leq c \leq 0.25 b_{b r}$
AISC 358, Eq 5.8-3

where

$a=$ distance from face of the column to the start of the cut

$b=$ length of the cut

$c=$ depth of the cut 
In addition, $c$ should be estimated to be 40 to 50 percent of the beam flange removal to limit the moment of column face to be no more than 90 to 100 of the plastic moment capacity of the full beam section (IBC, 2015).

Note that the radius of the cut

$$
R=\frac{4 * C^{2}+b^{2}}{8 * C}
$$

2. The plastic section modulus at the center of the reduced beam section:

$$
Z_{R B S}=Z_{X}-2 c t_{b f}\left(d-t_{b f}\right)
$$

3. The probable maximum moment, $\boldsymbol{M}_{\boldsymbol{p}}$, at the center of the reduced beam section:

$$
M_{p r}=C_{p r} R_{y} F_{y} Z_{R B S}
$$

4. Probable maximum moment at the face of column:

$$
M_{f}=M_{p r}+V_{R B S} S_{h}
$$

5. Check the flexural strength of the beam at the face of the column:

$$
M_{f} \leq \emptyset_{d} M_{p e}
$$

where

$\boldsymbol{M}_{p e}$ is the plastic moment of- the beam based on the expected yield stress.

And if equation from step 5 is not satisfied, the value of flange cut $\mathrm{c}$ needed to be adjusted and iterations are needed to get the most optimal flange cut percentage.

\subsubsection{Welding and Detailing of RBS for Special Moment Frame System}

For this project, the modeling specimens with RBS are anticipated to have welded beam web-column flange connection. Additionally, this type of welding method is 
believed be more effective compared to bolted connection for steel moment frame design (Engelhardt el at.,1998). Furthermore, modeling of fully welded connection in Strand7 can reduced the possibility to develop unpredictable stress due to the variable in bolt joint modeling method (Strand7 Reference Manual, 2015). As a result, the welded connection is also suitable for performing the design and analysis investigate the RBS for SMF system. The typical detailing of RBS design is shown in Figure 2.6, according to the International Building Code (2015). Note that the plate with bolted connection used in the beam and column joint. Additionally, it states in Chapter 5.6 from AISC 358 (2016), for the CJP groove weld, it extends between the weld access holes for the beam web to the column flange connection. In addition, the shear tab plate, as indicated in Figure 2.2, shall be at least $3 / 8 \mathrm{in}$. However, for the experimental testing program conducted by Dr. Engelhardt (1998) et al, fully welded connection was used in the specimen design because the stress level at the beam flange and flange grooves get to be reduced with weld method which was with improve toughness.

\subsection{Analytical studies with Finite Element Method (FEM) of Steel Moment Resisting Frame with RBS connection}

After the 1994 Northridge Earthquake, most experimental testing and numerical simulation were conducted for RBS's behavior for the moment connection only, but there were limited system level studies of MRF with this type of connection. Yet The need to 
understand the behavior of the beam to column connection on the moment resisting fame (MRF) has been recognized especially after the quake. The modeling method is crucial for studying effect of the hysteresis of structural response of MRF. The early research conducted with improved connection design had conducted by Researcher Brunesi et al. (2014) presented a model procedure for bolted connection which will be potentially used in MRF by using a nonlinear finite element modeling (FEM) platform. Critical behavior condition including Influence of friction, pretension of bolts and highly nonlinear contact elements etc. had been modeled to develop the cyclic-reverse test protocol. Bruneis et al. stated that the response produced by FEM was validated by the experimental numerical testing result.

To show that MEF modeling is a reliable modeling method for steel moment frame connection. Froster et al. (2016) investigated the accuracy of the new FEM approach to predict the behavior of the bolted moment connections between cold-formed steel members and software ABAQUS was used as the modeling platform. The study results showed that the three-dimensional shell elements model from were the most accurate prediction of hysteretic and energy capacity of the connections. Researcher Jablonska-Krysiewicz (2015) also proved that the modeling technique form FEM was capable to predict connection response to an acceptable degree of accuracy. Therefore, FEM is the good potential modeling method to make Real Time Hybrid Testing to be more feasible for building structural experimental testing, as Jablonska-Krysiewicz pointed out that the result can be used in advanced structural studies of beams and frames. 
Besides directly modeling the structure with FEM, there are studies incorporated FEM into modeling procedure to simulate and evaluate the performance of the steel moment frame connection for design purpose. Seif et al. (2016) presented the development of detailed element-level models of typical moment connection for steelframe structure and analytical method needed to simulate the structural connection subjected to realistic fire. The researchers from this study pointed out that framing structure would subject to large and unanticipated deformation during fires and leads to building collapse, and temperature-dependent material were able to be modeled in the FEM and dominant failure modes were identified after the model are calibrated against experimental test data. As a result, it proves that FEM is the suitable modeling method so far to leverage the analytical results with experimental results.

Afzalan and Ghassemieh (2015) applied the Cyclic Void Growth Model to predict the initial crack behavior and following fracture of steel moment connection. FEM by using software ABAQUS was used to assess the applicability of this model and the researchers stated that the results were reasonably consistent with the experimental test. A FE code from the study was utilized to simulate the initial cracking behavior during ultra-low fatigue. In this research project a newer FEM software named software Strand7 will be used to model the Reduced Beam Section moment frame, and the analytical experiment will be done using cyclic loading to attain the test data in order to compare the data from the previous similar research.

Jin et al. (2004) performed nonlinear push and transient analyses of 4-,8-,16- story frames respectively with RBS in order to develop a better understand of RBS frame behavior and evaluated the FEMA-350 (2000) design specification. In the study, the 
frames were analyzed using the computer program DYNAMIX (El-Tawil et al, 2001) and the capability of the RBS frame developing good seismic performance for high risk region was confirmed by the studies. Note that P-Delta effect and Geometric Nonlinearity also were accounted in the model. More Recently, researchers Naughton (2017) et al. performed a series of non-linear static push over analysis on steel moment resisting frames from Jin et al.'s research by using software ETABS. Moment rotation curve was generated and used to define the behavior of the seismic resistant connections. Different connection types including RBS were studied to compare the capacity curve difference.

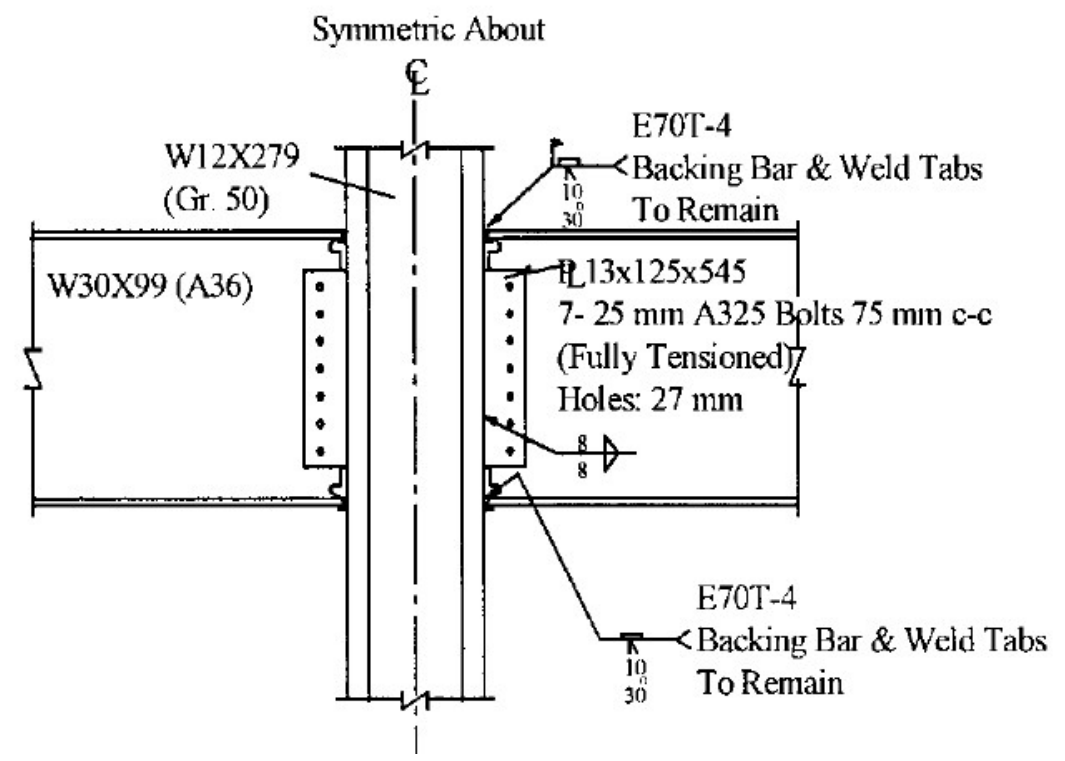

Figure 2.1 Pre-Northridge Connection Detail (Civjan et al. 2000) 


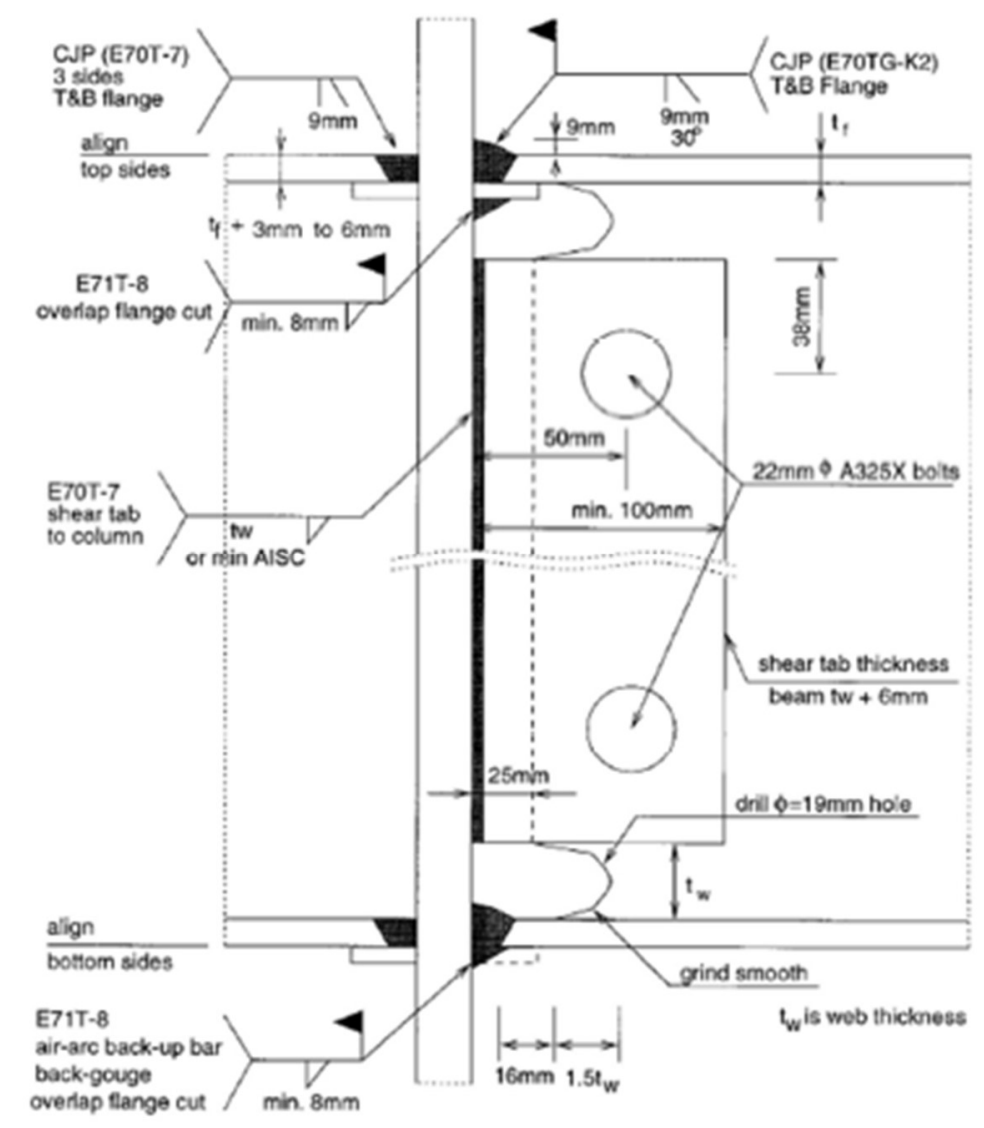

Figure 2.2 Post-Northridge Connection Detail (Stojadinovic et al. ,2000)

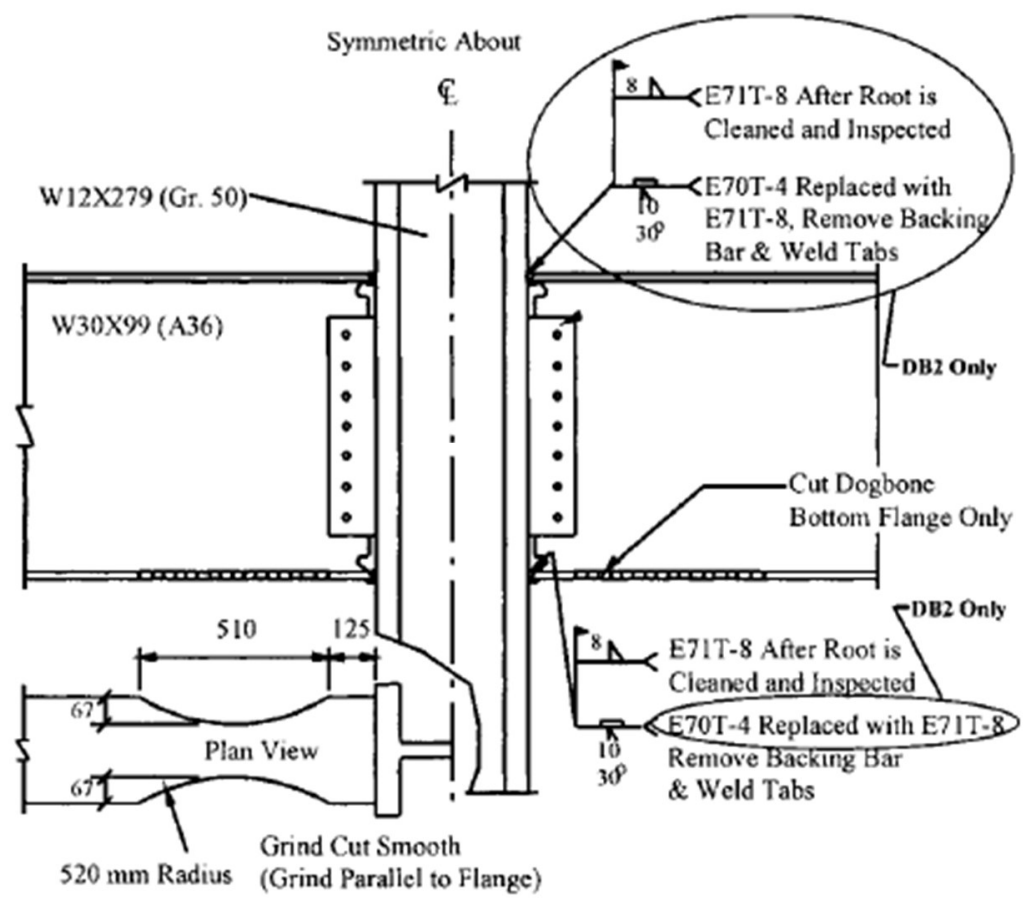


Figure 2.3 Post-Northridge Connection with

Dog-bone Retrofit Detail (Civjan et al., 2000)

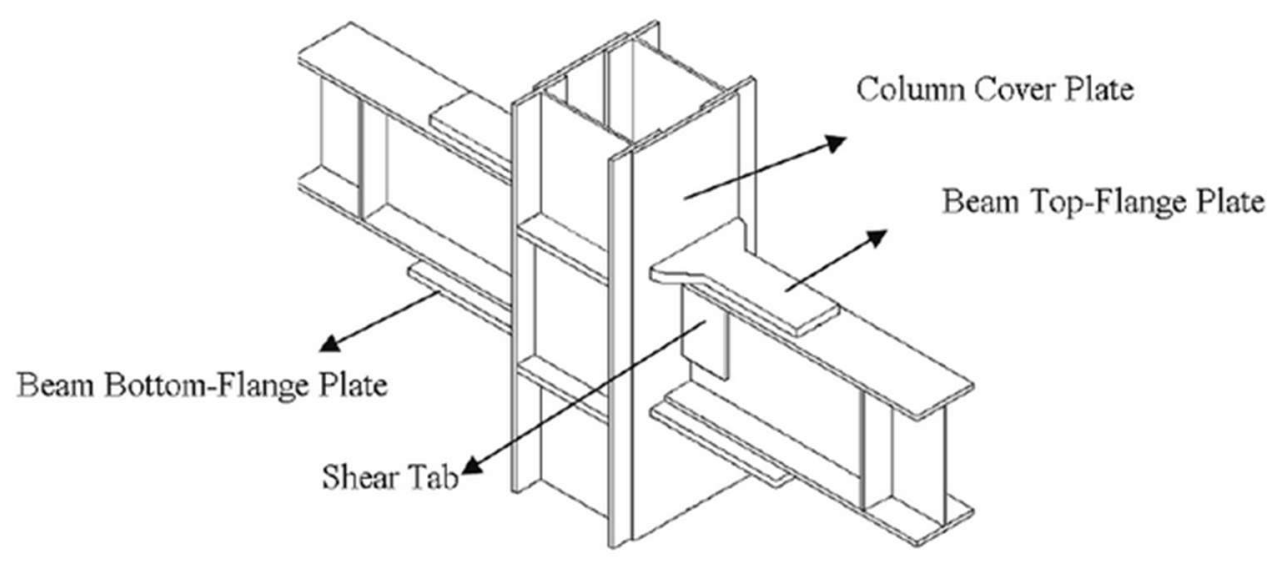

Figure 2.4 Cover Plate Connection (Deylami \& Gholipour, 2011)

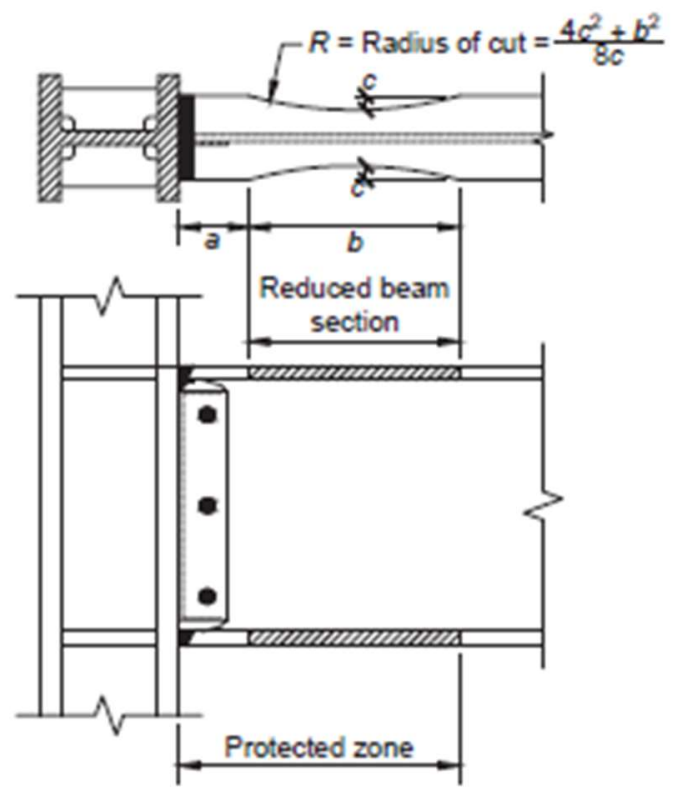

Figure 2.5 Reduced Beam Section Connection (AISC 358,2015) 


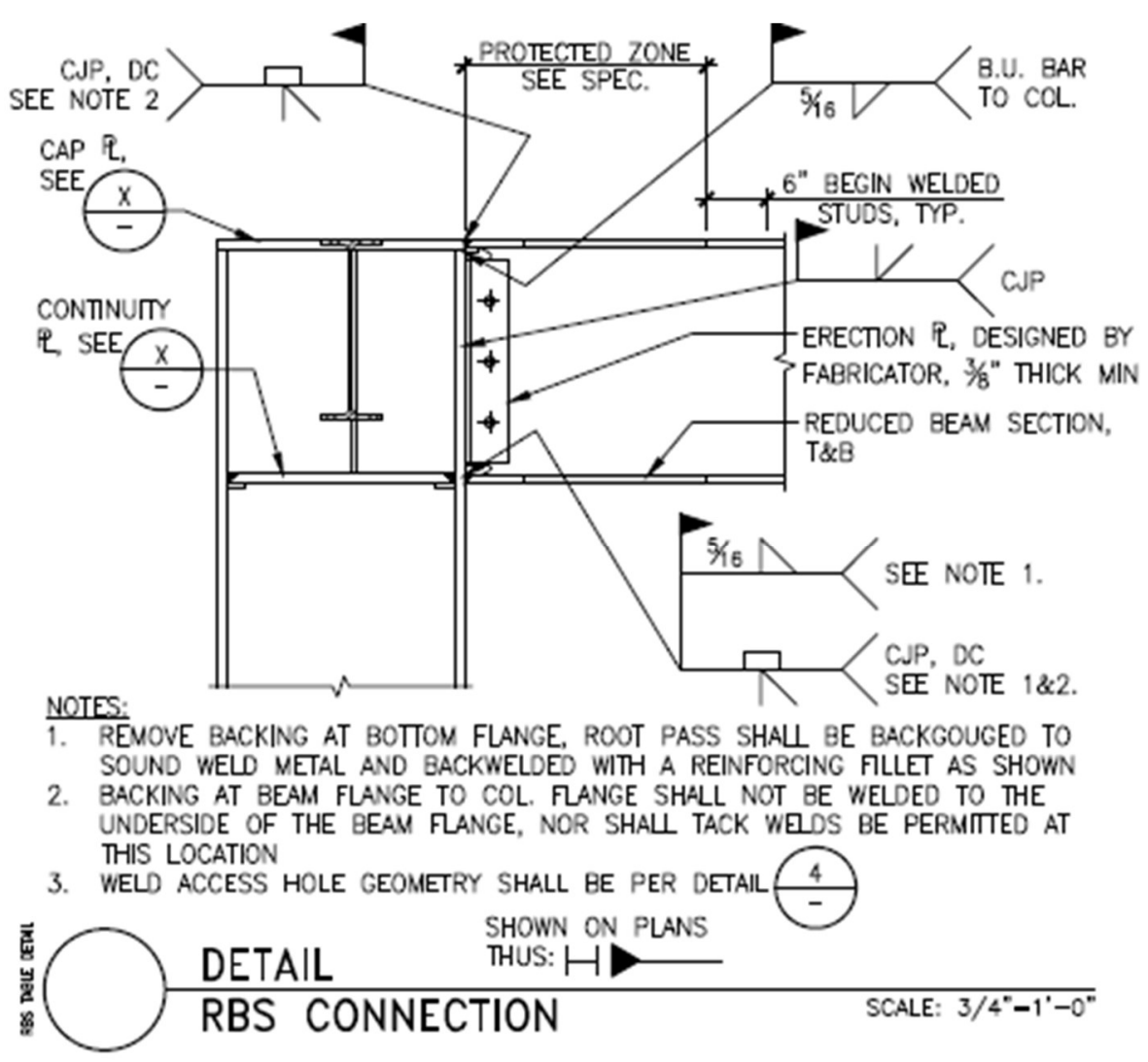

Figure 2.6 Detailing of RBS Design (2012 IBC, volume 4) 


\section{Chapter 3}

\section{Finite Element Modeling and Analysis of RBS connection}

\subsection{General Model Description}

The finite element study includes modeling the different types of RBS connection to investigate the nonlinear hysteresis response of the specimens. The nonlinear finite element analysis (FEA) program Strand7 was used to develop the 3 D nonlinear finite element models of connections. The model geometry including section sizes and span lengths which were closely follows the test setup from the test program. Furthermore, boundary conditions on the models were created based on the same experimental restraint condition with the test specimens.

A dog-bone radius cut RBS and constant cut RBS with a W14 column beamcolumn connections are chosen to be the model configurations. These two types of RBS connections were used to conduct the test program to investigate and evaluate the connection type to be used in an office building construction after the Northridge Earthquake (Engelhardt,1999). The details of both RBS connections are shown in figure 3.1 and Figure 3.2 accordingly. They are both exterior local connections from a moment frame system with cantilever beam connected to the flange of column. The continuity plates were Grade 36 steel and they were with approximately the same thickness as the 
beam flange. Studies were conducted by altering the details in the base model, as a result to create other finite element models. Models of moment connection with full beam section, were also made to compare the results in order to show the seismic performance of the prequalified RBS connection. Cyclic pushover analysis was performed to all the models.

\subsection{Finite Element Models of RBS with Strand7}

The modeling matrix for each model can be found in table 3.1. The member section sizes for the models in the analysis matrix were based on the member sizes which were selected for the test program. As mentioned by Dr. Engelhardt (1998), the DB1 Model has a bigger beam size W36x160 compared to DB2 with radius cut W36x150. Seismic performance of these two types of Dog Bone RBS connections are compared to investigate how the geometry of the RBS cut affected the response. Moreover, a completely joint penetration (CJP) welded web connection which described previously, was used for all models, due to the anticipated reliability of the weld web connection. Therefore, it reduced the stress level at both the beam flange and groove weld.

The models are created by using plate and brick elements form Strand7's element library accordingly, in order to investigate the nonlinear behavior response with different elements type to achieve the best suitable modeling and analysis method. Base on the capacity of the computer and mesh convergence sensitivity, plate models were efficient to show the general nonlinear response of the RBS. Furthermore, due to plate models' shorter solution time requirement and good mesh convergency with lesser number of 
elements compared to bricks, parametric studies were performed with the plate models. However, plate elements only use the in-plane stress components in the nonlinear material calculations and possibly has virtual overlap which cannot be avoided. Therefore, it might not produce results that fully agree with the test results. While the brick elements use the full stress tensor for the equivalent stress result in the nonlinear material calculation, hence the brick mesh should produce results that show better correlation with the test results (Strand 7 Reference Manual, 2020).

Analysis were conducted by using nonlinear static solver from Strand7 for models with plate and brick elements accordingly. Convergence of the model can be monitored from convergency graph of the solver window as shown in Figure 3.3. Because modeling with brick elements leads to great size of mesh that require approximately 3 times longer solution time compared to plate elements modeling, the convergency graph was used to determine the appropriate layers of bricks to create the model with the correct bending behavior of the beam in the RBS connection.

\subsubsection{Plate and Brick Global Model}

The test set up from the test program for the connection specimens is shown in Figure 3.4. An idealized model of the test setup for the connection specimens also are shown in Figure 3.5 for clarity to use in FEA. The span length from the column face to the beam end reaction was $134 \mathrm{in}$, and the length between the top roller support of the column and the pin at the bottom was 136in. Lateral bracings were applied to the beam 84 in away from column face. 
The typical 3-D global models of RBS for FEA were shown in Figure 3.6 to 3.7 with plate elements and brick elements accordingly. Note that the general size of the geometry of the cut were based on the details shown in Figure 3.1 and 3.2, while the radius of the cut of DB2 model was based on the design equation noted in figure 2.3 with $\mathrm{R}=\frac{4 c^{2}+b^{2}}{8 * c}$, according to code AISC 358 (2016) provision, as described previously. The plan views of FEA models show the constant cut RBS and radius cut RBS geometries accordingly in Figure 3.8 and Figure 3.9.

\subsubsection{Models with Plate Element}

Plate element from Strand7 element library is two-dimensional surface element. A preliminary model is created with plates to investigate the general behavior of the RBS due to the relatively shorter solution time from the nonlinear static solver. Two types of plate elements Quad4 and Quad 8 were selected to create the models, as shown in Figure 3.10. Quad 4 was only suitable for modeling geometry without radius. Therefore, Quad 8 was used in DB2 models to generate the curved beam flange with the right radius cut reduction. Addition modeling step by creating user defined coordinates to locate the center of the Dog-Bone radius to determine the appropriate radius length with beam elements. Nevertheless, the beam elements were deleted to keep the number of elements and degree of freedom in the anticipate suitable range for the mesh convergence. 
The Plate/Shell from plate element property use isotropic hardening model for material and it is appropriate to use for modeling steel material because it assumes that it has the same elastic modulus in all directions. In addition, a shell element is more capable of capturing the effect of local buckling (Zhang, 2004). Models with full beam section were also created to generate results to compare with DB1 and DB2 models to show the seismic performance of this prequalified moment connection subassemblies. The mesh for a typical global model consisting of a W14x426 column and a W 36x150 beam had a total of approximately 3217 nodes, 3096 plates and resulting in 18984 equations. Note that each degree of freedom from an element outcome an equation respectively.

\subsubsection{Modeling with Brick Element}

Brick element from strand 7 library is three-dimensional solid element. Modeling with brick element requires consideration of mesh convergency as well as computer capacity due to the high degree of freedom equations with the big numbers of nodes in brick models. FEA models were created using brick element to attain more accurate results with more detail information. Similar to plate element modeling as mentioned above, models with full beam section were also made to perform comparison analysis.

Brick element has 3 degree of freedoms of each node. To model the nonlinear bending behavior, Hexon 8 brick element type was use and the bubble function was incorporated in the element property to improve performance. Bubble function introduces compatible displacement modes into the element. These modes are internal degree of 
freedom that don't need to define additional nodes (Strand7 Reference Manual, 2020). DB1 and DB2 brick models have different numbers of nodes and elements because the geometry of the RBS for each model was different. Apparently DB2 consisted more nodes resulting approximately 23974 equations and it was almost $30 \%$ more number of equations than the plate models.

\subsubsection{FEA Models’ Boundary Condition and Loading Protocol}

There were roller and pined boundary conditions in the global model, as mentioned in the above session, in order to achieve the same boundary condition which was used in the experiment testing. The roller boundary condition was achieved by restraining the translational displacement along both the $\mathrm{X}$ and $\mathrm{Y}$ direction in the global coordinate. The pin boundary condition was modelled by restraining all the translational displacement and rotations, except for the rotation about $\mathrm{X}$-axis, which is normal to the

plane of the column, as shown in Figure 3.11. Therefore, the model would have a deform shape shown in Figure 3.12 according to the one defined in FEMA 350. Additionally, in order to simulate the lateral-torsional bracing for the experimental set up, out of plane movement of the beam was restrain at the flange in 84 in from the column face.

The FEA were conducted by applying cyclic displacement with variable amplitude at the beam end. The target amplitude was achieved by assigning a unit displace to the beam end and load increments for this freedom case was set up in the nonlinear static solver. Instead of applying unit displacement to every node of the beam 
end, a rigid link connection was to slave all the end notes into a master node. As a result, the displacement only needs to be applied to the master node and it is convenient to extract reaction force from the master node with even distribution of displacement without having a flexible end plate, according to Strand7 Reference Manual (2015).

The cyclic displacement at beam end followed the loading protocol suggested by a report from Applied Technology Council (1992), which was used in the experimental test program (Engelhardt el at. 1998). A table of this loading protocol is shown in Table 3.2 and a graphic presentation of the displacement loads are shown in Figure 3.13. The loading protocol was set as a freedom case and applied at the respective master node of the beam tip in the model as mentioned above. Here the freedom case is a translational displacement on global vertical direction (global Z) instead of a force load. It is displacement-controlled loading history. By restraining the master slaved node with setting it as a 1.0 enforced displacement along $Z$ direction, while controlling all other restraint to match testing setup, the freedom case will scale the 1.0 displacement to the drift displacement interval per each loading cycle during analysis.

The magnitude of the cyclic displacement loading was set up in the nonlinear static solver with an increment of 0.5 inches by following the respective loading protocol as mentioned. There were 547 total increments and Displacement Scaling was used in the sub-step automatic sub incrementation to improve convergency. According to Strand7 Reference Manual (2020), Displacement Scaling is a load scaling method that scales down the calculated displacements to keep them withing the pre-set limits and the load lost is treated as an additional residual in the next iteration of the increment, therefore, the load step eventually converges. 
On the other hand, researchers Jiao and Yamada (2018) pointed out that the effect of different loading protocol can lead to different result from the analysis. However, in order to compare with the experimental results, and to verify the reliability of the modeling method, the protocol from the testing program was used.

\subsubsection{Von mises Stress Yield Criterion}

For the material property definition, yield criterion needs to be defined to monitor yielding in the analysis. The Von Mises stress yield criterion is generally applicable to metal plasticity (Strand7 Reference Manual,2020). Based on the yield criteria the Von Mises Stress $\sigma_{\mathbf{v m}}$ can be defined as:

$$
\sigma_{v m}=\sqrt{\frac{1}{2}\left(\left(\sigma_{11}-\sigma_{22}\right)^{2}+\left(\sigma_{22}-\sigma_{33}\right)^{2}+\left(\sigma_{33}-\sigma_{11}\right)^{2}\right)}
$$

where $\sigma_{11}, \sigma_{22}, \sigma_{33}$ are principal stresses such that $\sigma_{33} \leq \sigma_{22} \leq \sigma_{11}$.

Once Von mises yield criterion is defined in the material for nonlinear definition, Strand7 uses yield criterion function to monitor yielding in a material. We defined steel as elasticplastic material that is ductile, and Von Mises Criterion is applicable for modeling with this type of material. As a result, Von Mises Stress Yield Criterion was setup in the plate and brick properties for all the models herein and FEA results from the following sections were generated based on this yield criterion. In addition, a direct sparse scheme which is a high-performance solver that is suitable to solve for big size of model, was selected in the nonlinear static solver. Direct Sparse solver allows very fast solution of large systems 
of by utilized the sparsity of the matrix. A matrix is sparse if the number of non-zero entries is small compared with the total number of entries in the matrix (Strand7 Webnotes,2010).

\subsubsection{Material Properties}

The material property from Tensile Coupon test for the beams, columns in each model is defined based on the material used in the specimen experimental testing which was mentioned by Dr. Engelhardt et al. (1998), which is shown in Table 3. Note that there the tensile coupon test data were not available for DB1 column. As a result, the columns from models of DB1 were assumed to have the same properties of DB2 columns. Furthermore, note that the yield and tensile strength for the web and flange of the section were different. Consequently, 4 different individual Stress vs Strain-curve was defined in strand7 shown in Figure 3.14 to 3.17 to capture the distinct nonlinear material properties of the flange and web for the column as well as beam sections. Since the yield strain and ultimate strain were available from tensile test data according to the experiment, a stress vs strain table for typical mild steel which was obtained from Strand7's table library was used as a reference to define the corresponding strain for yielding and fracture.

The material property of the shear tab and the continuity plates was assumed to follow the relationship of the typical mil steel stress vs strain curve which mentioned above. At the same time, the material was considered a von Mises material and strain 
hardening was included in the stress strain relationship as well as following nonlinear isotropic hardening (Zhang, 2000).

The bill of materials for DB2 plate is shown in Figure 3.18 and the total mass of material for a typical model is around 6700 pounds.

Strand7 carries out a linear interpolation between adjacent points in the defined Stress vs Strain tables to determine the stress level for a given strain. As mentioned in the previous session, the models herein used steel, which is an elastic plastic material. This type of material allows for a nonlinear relationship between stress and strain, including the development of plastic, or permanent deformation upon the cyclic unloading. According to Strand7 Reference Manual (2020), reverse loading takes a different path to that of loading and as a result, the material considers hysteresis effects from repeated loading-unloading-reloading cycles. In addition, tensile behavior may be different to compressive behavior depending on the shape of the table. Therefore, the defined Stress vs Strain curve might affect the nonlinear behavior of the RBS connection and generate a solution that depicted difference of results from the one attained from experimental testing.

For the plate elements modeling for FEA in Strand7, the layers of the plates play an important role in material nonlinear analysis. In the plate models for this project, the number of layers of the beam sections were set to be 50 while the layers of the column sections were set to be 20 since the number of layers used can influence the progression of yielding through plate thickness. Nevertheless, the onset of yielding is not very sensitive to the number of plate layers in Strand7 because a very thin layer was added to 
both surface of the element to help with detecting the onset of yield. Therefore, more layers were used for the beam sections because it was assumed that there were more yielding and bending behavior happened in the beam section during the cyclic loading simulation. Furthermore, according to Strand7 Reference Online Help (2020), the plate members are acting as plate shell type and a better representation of the yielding will achieve when more layers are used in plate element modeling, but with increasing of the analysis solution time. As a result, high number of layers were used in beam section only, while the column section was with less layers.

\subsubsection{Geometric Nonlinearity in Finite Element Analysis}

Geometric nonlinearity was considered in the analysis that the stress-strain table is interpreted differently from engineering stress-strain values. As the stress and strain data is calculated using the original cross section area and length of the specimen based on engineering stress-strain tables, when geometric nonlinearity was not including for analysis, the solver would not update the geometry as the structural specimen deformed. Nevertheless, herein the true stress is calculated by using the actual cross section area at the point where the measurement was taken during the nonlinear analysis, because geometric nonlinearity was used. Refer to Strand7 Webnote (2020), the relationship between the true stress-strain curve and the engineering stress-strain curve follows from equations:

$$
\sigma_{T}=\sigma_{E}\left(1+\varepsilon_{E}\right)
$$




$$
\varepsilon_{T}=\operatorname{Ln}\left(1+\varepsilon_{E}\right)
$$

where

$\sigma_{\mathrm{T},} \varepsilon_{\mathrm{T}}=$ true stress, true strain

$\sigma_{\mathrm{E}}, \varepsilon_{\mathrm{E}}=$ engineering stress, engineering strain

Figure 3.19 show the discrepancy of the stress and strain data from the analysis and the input stress-strain curve when the strain was over $0.03(3 \%)$ with geometric nonlinearity included in the analysis for a typical brick element model (referred to Strand7 Reference Manual). Note that the data from analysis result were with higher stresses than the defined stresses data, as the strain increased.

In the plate models, the plate/shell element which was used in geometric nonlinear analysis considers large displacements but still assumes that strains were small. While for elements based on large strain formulations (e.g. brick elements), the element formulation may require other strain definitions to ensure that the stress and strain measures used form an appropriate pair, according to Strand7 Reference Manual (2020). Geometric Nonlinearity becomes larger when changes in geometry or P-Delta effect increases, which have a significant effect on the load deflection characteristics such as stiffness of the structure (Strand7 Webnotes,2015). Therefore, higher stress was assumed to develop in the model in the nonlinear static analysis, and the stress vs. strain curves for a separate brick model was created with modified stress vs. stress curve with lower stress magnitude compared to the original stress vs strain data which was defined for the plate models. The result of this brick model will be discussed in the following session. 


\subsection{Finite Element Analysis Results}

Finite element nonlinear static analysis results from both plate models and brick models are presented here and compared to experimental results to verify the selected element type and mesh size for the global models. Additionally, the geometry of RBS, axial load to the connection are investigated with the plate models as well. Since it is convenient to change parameters of the connection with plate models because of its smaller numbers of elements, the parameters studies were all performed with plate RBS models.

\subsubsection{FEA Plate Models Results}

As mentioned previously, DB1 and DB2 were exterior welded beam to column flange connection with RBS tested in the program referred to Dr. (1998). Both connections were modelled with the FEA software Strand7 by using the modeling techniques and the selected plate element type. The analysis results of the RBS connections models were compared to the result of full beam column connection, to examine the increase of ductility effect from RBS to the behavior of the structure. In addition. the analysis results were compared to the experimental results to verify that RBS is with good ductile performance together brings economic design.

The hysteresis response of each specimen which were modelled with plate elements and brick elements were generated from Strand7. The idealized plastic hinge location is assumed to be at the minimum section of RBS. First type of response is the 
beam load versus deflection plots. The beam load is extracted from Strand7 by plotting the reaction along $\mathrm{Z}$ direction at the beam tip master-slaved node. Note that autoassigning a rigid connection was applied on all the nodes of beam end of the specimen and the master-slaved node was chosen to attain the results which represents all the reactions of beam end nodes, as mentioned previously. Deflection is measured by taking the displacement of the master-slaved node.

Second type of response is the beam moment versus rotation plots. The beam moment is measured at the minimum section of reduced beam section and computed by taking the beam tip Fz reaction force from the mastered slaved node, multiplied by and distance to the RBS minimum section (116 inches). The rotation was computed by taking the deflection of the beam tip and divided by the distance to the minimum RBS section. Therefore, both moment and rotation showed in figure 15 and 16 are computed respective to minimum section of the RBS. Experimental results are also included in these plots to show the reliability of the modeling method.

\subsubsection{DB1 Constant Cut RBS Connection Model FEA Results}

By inspecting the Load vs. Deflection analytical results showed in Figure 3.20 and 3.21 , specimen DB1 which was with a constant width cut, showed good performance in the early stage of the cyclic loading simulation and it also was consistent with response from the experimental testing as indicated in the graph. Nevertheless, the experiment was stopped before the rotation of 0.04 radian due to the limitation of the test setup in order to avoid damage of the equipment. Analytical results with FEA was able to run the simulation to over 0.05 rad to inspect the hysteresis response of the specimen in 
the later inelastic cycles. In addition, by comparing the analytical results with the experimental curve in Figure 3.20 the DB1 FEA plate model accurately predict the elastic stiffness and inelastic stiffness of the specimen and hysteresis nonlinear response of the specimen during the seismic cyclic loading simulation up to 0.04 radian, which was when the experimental testing stop. Moreover, over around \pm 0.04 radian, the hysteresis response started to show clear degradation. As described by Dr. Engelhardt (1998) about the experimental test results, fracture of the beam flange occurred at the end of the testing and it was anticipated that was due to lateral torsional buckling which will be discussed and verified in the following.

Likewise, the DB1 in Figure 3.21 FEA brick model produced similar hysteresis response for the specimen under cyclic loading, nevertheless it showed less degree of deterioration compared to the DB1 plate model. One possible reason is that there is one brick element through the thickness the flange and that may not only increase the stiffness of beam and flange but also the stress and therefore the yielding behavior, according to Strand7 Software support (2020). It is recommended to use more at least 4 brick through the thickness of the webs in order to model better yielding and bending behavior. Notwithstanding, with increasing of brick elements, mesh size will become large to solve and due to limit computer CPU capacity, further investigate of the mesh convergency studies is not conducted herein. Yet overall, the brick model is adequate to inspect the nonlinear response of the specimen.

At the end of the simulation, the FEA mode show high stress level (313.7 ksi) at around minimum section of the RBS, which indicated in the Von Mises Stress contour 
plot in Figure 3.22. Contrarily, at the beam column connection location showed less stress level (66 ksi) compared to the minimum section of RBS. As a result, it clearly showed the effect of RBS which moved the plastic hinge away from the column beam connection and forced it to form in the beam. In other words, plastic deformation would mostly happen in the beam instead of the column. Furthermore, the investigation was conducted by using the FEA DB1 brick model as well. The stress level in the brick model also show comparable indication of plastic hinge formation near the RBS region. As shown in Figure 3.24 of the contour plot of DB1 brick model, yet the maximum stress is $167 \mathrm{ksi}$, which is lower than the result from the plate model due to the bigger stiffness of the brick element modeling effect.

\subsubsection{DB1 Beam Local and Lateral Deformation Studies}

In order to investigate more about the effect of the RBS, the result from the relevant plate model with the full beam section were extracted to compare with DB1 plate models' results. As shown in Figure 3.27 (b), at the end of the simulation with 0.05 radians rotation, there is significant yielding in the beam and propagate near the column face for the full beam section model. It was anticipated that if additional loading cycles were added to the simulation, the yielding will spread to the connection where the CJP groove weld usually is constructed in practice. From Figure 3.27(a), RBS showed good performance with the less sever yielding near the column face compared to the full beam section model. Von Mises stresses near the column face connection of the beam web is $94.3 \mathrm{ksi}$ for the full beam section model, while it is $66.3 \mathrm{ksi}$ for DB1 model. Hence, it that 
RBS reduces the stress concentration at the connection and the degree of yielding of the unreduced flange portion of beam flange near the column face.

Lateral out of plane movement of the beam were observed starting from the DB1 plate model along the increasing of cyclic loading. By plotting the lateral out of plane displacement of the beam flange for RBS minimum section of the connection and full beam connection showing in Figure 3.28, it suggests that local and torsional buckling of the beam occurred in the connections. The degree of bucking is greater in RBS flange with 2.6 in lateral out of plane displacement, compared to 1.4 in lateral out of plane displacement for the full beam section connection. Furthermore, buckling occurs in the beam web for both type of connections and it is shown in Figure 3.29. Note that the RBS beam web is with 3.78 in lateral out of plane displacement and the comparative web lateral displacement in full beam section connection is $5.57 \mathrm{in}$. As a result, these beam flange and web buckling induce addition stresses in the beam weaken region, and those high stresses were shown in the Von Mises stress contour plot in Figure 3.27.

Furthermore, the lateral out of plane displacement of the node from the beam web near the RBS respective to the displacement loading, were plotted in Figure 3.25 and 3.26 for both DB1 plate and brick model. Note that lateral displacement become greater as the cyclic loading with bigger magnitude. As a result, local flange and web buckling combined with lateral torsional buckling can be detected in the FEA model as shown in Figure 3.22 and 3.23 for DB1 plate and brick models. But the buckling of the beam is more severe as shown in the plate model compared to the brick model under the same 
$10 \%$ displacement display scale. The reason is that virtue overlap of plate elements can't be avoided in FEA modeling (Strand7 Reference Manual,2020).

In addition, the brick model overestimates the ultimate strength of the specimen which is shown in the moment versus rotation graphs of the beam, yet it accurately predicted the rotation increment where deterioration occurs which matches the experimental outcome. The target level of plastic rotation for acceptable performance for the test program was 0.03 radians, and it clearly is showing from the both plate model and brick models' beam moment versus rotation plots accordingly that the specimen DB1 was capable to develop the target rotation for its seismic performance, yet it show relatively degree of buckling when it reached the loading close respective to the end of the loading of the experimental testing.

As pointed out by researcher Zhang (2004) and Engelhardt (1998) that radius cut RBS connection had better seismic performance than other types of RBS, therefore, the need to get more useful understanding of the radius cut RBS is perceived by the FEA method and the results will be presented in the following to investigate the different nonlinear response between DB1 with constant cut and DB2 with radius cut beam section to corroborate the reliability and cost effectiveness of the later by changing the shape of the dog-bone cutout.

\subsubsection{DB2 Radius Cut RBS Connection Model FEA Results}

In order to justify the effectiveness of the radius cut geometry of the RBS, beam reaction versus deflection and moment versus rotation plots are revealed in Figure 3.30 to 
Figure 3.33. The specimen's ultimate load of beam end reaches over 200 kips, as indicated in both plate and brick FEA model. Furthermore, the response of moment versus rotation reveals that the specimen developed 0.03 radians of rotation before the significant strength deterioration occurs. Yet comparable results can be seen between the plane and brick FEA modeling. The brick FEA model over-estimates the ultimate moment developed in the center of RBS, which is showing the hysteresis loop in Figure Figure 3.33 with relative less percentage of deterioration compared to the plate FEA. It is possibly due to the assumption with the fully welded beam web without weld access hole modeled, therefore, it results higher stiffness that can lead to higher moment development during the nonlinear deformation occurrence period.

Moreover, the DB2 exhibit better ductile behavior with a wider hysteresis response with less severe beam flange and web local lateral buckling compared to DB1, not only presented in the experimental results, but also predicted by the FEA models shown in Figure 3.30 to Figure 3.33. Further investigation can be conducted to tune the parameters of FEA model to bring the discrepancy lower between analytical results and experimental results However, higher cost of design and fabrication will be required to produce the appropriate geometry of the radius cut RBS which give rise to promising seismic performance according to FEA model prediction.

\subsubsection{Beam moment analysis of RBS connection}

To further investigate the performance of the RBS, beam moments were shown in Table 3.4. The estimated plastic moment of each tested beam for both FEA plate models were calculated based on the yield stresses referred to the test program tensile coupon 
data of the sections' material. Note that the moment is presented for both full beam section and for the RBS where at the center of the flange cut. The results demonstrate the maximum moment developed within the RBS for each FEA model is 1.07 times the plastic moment of the reduced section for DB1 and 1.28 times for DB2. These values suggest that there was minor percentage of strain hardening takes place in DB1 dog-bone flange, while there is essential strain hardening in DB2. The moment in beam generates at the column face is 1.06 times the plastic moment of the full beam section for DB1, and 1.13 times for DB2. As a result, the welded beam to column connection used in the FEA modeling was adequate to withstand these moments by complying the strong column weak beam design concept.

\subsubsection{Effect of Axial Force for DB2 RBS Specimen}

To investigate the effect of axial load on the radius cut RBS connection, FEA analysis was done with the calculated axial load applied to the column of DB2 model before applying the seismic cyclic loading. Therefore, the model is subjected to axial load combined with earthquake load. Three cases with DB2 model member sections with varying axial load parameters was analyzed. The column panel zone is anticipated to have the enough shear strength to resist the shear develop in the panel zone. The analysis matrix is shown in table 3.5 and continuity plate thickness is 1 in according to the experimental setups. By assuming the material of the column is with $50 \mathrm{ksi}$ nominal yield 
strength, herein we can calculate the reasonable axial load to use in the model by multiplying the gross section area of the column (125 in^2) with $50 \mathrm{ksi}$ to obtain the nominal axial capacity of the column in compression. There is no axial load applied to the column in case one, while case 2 and 3 have axial loads respect to $5 \%$ and $10 \%$ of column axial capacity, accordingly. Note that results kips of axial load applied to column in case 2 and kips of axial load applied to column in case 3. It is equivalent of $2.5 \mathrm{ksi}$ and $5 \mathrm{ksi}$ of column stress for case 2 and case 3 respectively.

The moment versus rotation for the various cases were shown in Figure 3.34. It can be seen from the figure that the axial load doesn't alter the nonlinear response of the specimen under cyclic loading significantly. Though the stiffness of the specimen got alter slightly due to the applied amount of axial load, the specimen still develop about 0.03 radius of rotation before strength deterioration occurs. Therefore, it shows the column (W14x425) has adequate strength to resist the applied axial load ( up to 10\%) and the plastic hinge is formed in the RBS beam section, as shown in Figure 3.35. Yet it can be foreseen that with increasing magnitude of axial loads applied to the column, the specimen will experience fracture with a faster rate because of the weakening strength of column. Yield Index contour plots for the three cases are show Figure 3.23 to show the degree of yielding of the column for the specimen when the cyclic loading reached 0.05 radians rotation ( $=6$ in displacement load). Prediction of failure of the column can be made by inspecting the yield index on the column. In case 1, there is yielding of column panel zone but yield of top and bottom column section with no axial load applied. For case 2 and 3 , significant yielding of the column to and bottom section plus panel zone yielding can be detected. Additionally, there are relatively more area of beam yielding 
near the connection showing in the case with axial load applied. Besides, yielding along the beam lateral bracing can also be seen. As a result, the degree of instability of the DB2 connection can be efficiently predicted in Strand7 FEA method.

Table 3.1 Modeling Matrix

\begin{tabular}{|c|c|c|c|c|}
\hline Specimen & Beam Size & Column Size & $\begin{array}{c}\text { Loading } \\
\text { Protocol }\end{array}$ & $\begin{array}{c}\text { Flange } \\
\text { Reduction } \\
\text { Ratio }\end{array}$ \\
\hline DB1 & W36x160 & W14x426 & $\begin{array}{c}\text { Modified } \\
\text { ATC-29 }\end{array}$ & $39.6 \%$ \\
\hline DB2 & W36x150 & W14x126 & $\begin{array}{c}\text { Modified } \\
\text { ATC-29 }\end{array}$ & $39.3 \%$ \\
\hline
\end{tabular}

Table 3.2 Loading History for Specimens (Modified)

\begin{tabular}{|c|c|}
\hline & \\
\hline Beam Tip Displacement (inches) & Number of Loading Cycles \\
\hline \pm 0.5 & 3 \\
\hline \pm 0.75 & 3 \\
\hline \pm 1.0 & 3 \\
\hline \pm 2.0 & 3 \\
\hline \pm 3.0 & 2 \\
\hline \pm 4.0 & 2 \\
\hline \pm 5.0 & 2 \\
\hline \pm 6.0 & \\
\hline
\end{tabular}


Table 3.3 Connection Sections Material Properties from Coupon Tensil Test

(Engelhardt, 1998)

\begin{tabular}{|c|l|l|c|c|c|c|}
\hline \multirow{2}{*}{ Specimen } & \multirow{2}{*}{ Beam } & \multirow{2}{*}{ Column } & \multicolumn{2}{|c|}{ Yield Stress (ksi) } & \multicolumn{2}{c|}{ Tensile Strength (ksi) } \\
\cline { 4 - 7 } & & & Flange & Web & Flange & Web \\
\hline DB1 & W36x160 & & 54.7 & 53.5 & 75.6 & 79.2 \\
\hline & & W14x426 & NA & NA & NA & NA \\
\hline DB2 & W36x150 & & 41.4 & 47.1 & 58.7 & 61.8 \\
\hline & & W14x426 & 49.9 & 50.0 & 74.5 & 75.0 \\
\hline
\end{tabular}

Table 3.4 Plate Model Information of DB1 and DB2

\begin{tabular}{|c|c|c|c|c|c|c|c|}
\hline & $\begin{array}{c}\text { Element } \\
\text { Number }\end{array}$ & $\begin{array}{c}\text { Node } \\
\text { Number }\end{array}$ & $\begin{array}{c}\text { Model } \\
\text { Mass } \\
(\mathrm{lb})\end{array}$ & $\begin{array}{c}\text { Number } \\
\text { of } \\
\text { Equations }\end{array}$ & $\begin{array}{c}\text { Number } \\
\text { of Link }\end{array}$ & $\begin{array}{c}\text { Maximum } \\
\text { Bandi }\end{array}$ & $\begin{array}{c}\text { Average } \\
\text { Bandi }\end{array}$ \\
\hline DB1 & 3133 & 3024 & 6646.25 & 18528 & 22 & 621 & 128 \\
\hline DB2 & 2880 & 2987 & 6546.71 & 17676 & 22 & 539 & 122 \\
\hline NONDB1 & 3064 & 3177 & 6768.73 & 18792 & 22 & 671 & 129 \\
\hline NONDB2 & 2880 & 2987 & 6592.30 & 17676 & 22 & 611 & 127 \\
\hline
\end{tabular}

Table 3.5 Beam Moments of DB1 and DB2 (FEA Plate Model)

\begin{tabular}{|c|c|c|c|c|c|c|c|c|}
\hline $\begin{array}{l}\text { Specimen } \\
\text { Beam }\end{array}$ & & $\begin{array}{l}F y \\
\text { (ksi) }\end{array}$ & $M_{P}(k i p-i n)$ & $\begin{array}{l}M_{P-R B S} \\
\text { (kip-in) }\end{array}$ & $M_{\max }($ kip-in) & $\begin{array}{l}M_{\max -R B S} \\
\text { (kip-in) }\end{array}$ & $M_{\max } / M_{p}$ & $M_{\max -R B S} / M_{p-R B S}$ \\
\hline \multicolumn{9}{|l|}{$\begin{array}{l}\text { DB1 } \\
\text { W36X160 }\end{array}$} \\
\hline & Flange & 54.7 & 23420 & 18784 & & & & \\
\hline & Web & 53.5 & 10026 & 10026 & & & & \\
\hline & sum & & 33446 & 28810 & 35636.42 & 30849.43 & 1.06548 & 1.0708 \\
\hline \multicolumn{9}{|l|}{$\begin{array}{l}\text { DB2 } \\
\text { W36X150 }\end{array}$} \\
\hline & Flange & 41.4 & 16324 & 10493 & & & & \\
\hline
\end{tabular}




\begin{tabular}{|l|l|r|r|r|r|r|r|r|} 
& & & & & & & \\
\hline Web & 47.1 & 8516 & 8516 & & & & \\
& sum & & 24840 & 19009 & 28161 & 24379 & 1.13373 & 1.2825 \\
\hline
\end{tabular}

Note: $\quad M_{p}=F_{y} * Z_{x}$

$M_{p-R B S}=F_{y} * Z_{R B S}$

$Z_{R B S}=Z_{x}-2 * c * t_{b f} *\left(d-t_{b f}\right)$

Table 3.6 Analysis matrix to evaluate effect of axial load on cyclic global performance of DB2 RBS connection

\begin{tabular}{|c|c|c|c|c|c|}
\hline Case & Column & Beam & $\begin{array}{c}\text { Doubler } \\
\text { Plate? }\end{array}$ & $\begin{array}{c}\text { Continuity } \\
\text { Plate } \\
\text { Thickness } \\
\text { (in) }\end{array}$ & $\begin{array}{c}\text { Stress due } \\
\text { to Axial } \\
\text { Load (ksi) }\end{array}$ \\
\hline 1 & W 14x426 & $36 \times 150$ & No & 1.0 & 0 \\
\hline 2 & W 14x426 & $36 \times 150$ & No & 1.0 & 2.5 \\
\hline 3 & W 14x426 & $36 \times 150$ & No & 1.0 & 5.0 \\
\hline
\end{tabular}




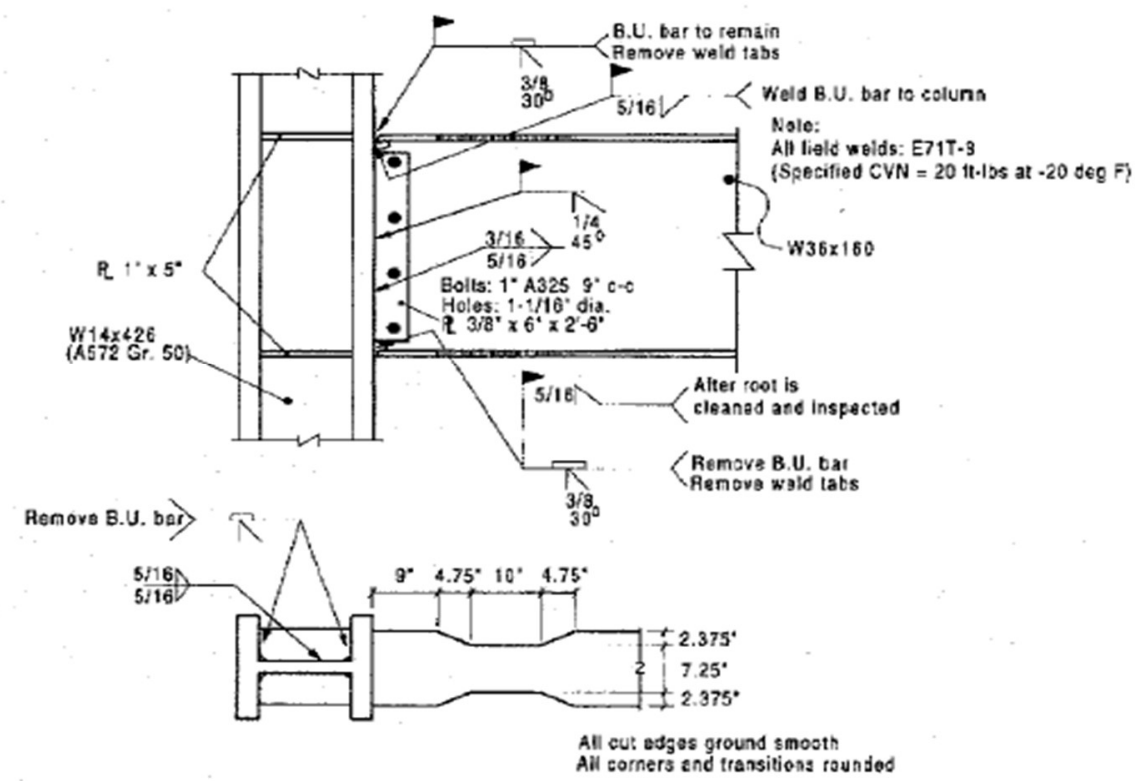

Figure 3.1 DB1 Dog Bone Connection Detail (Engelhardt et al. 1998)

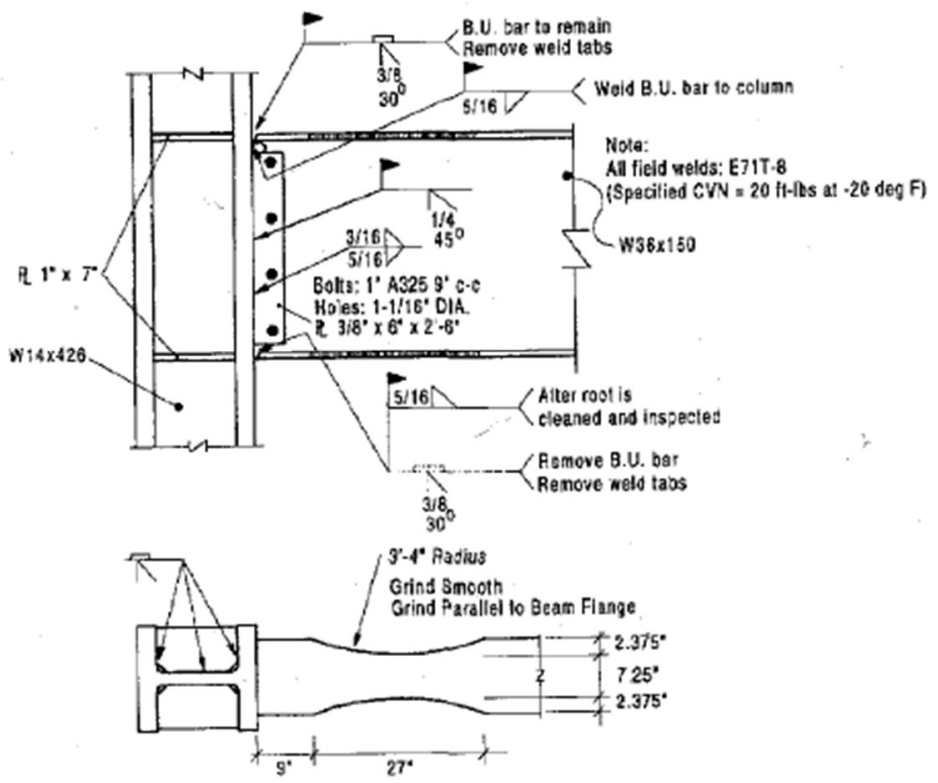

Figure 3.2 DB2 Dog Bone Connection Detail (Engelhardt et al. 1998) 


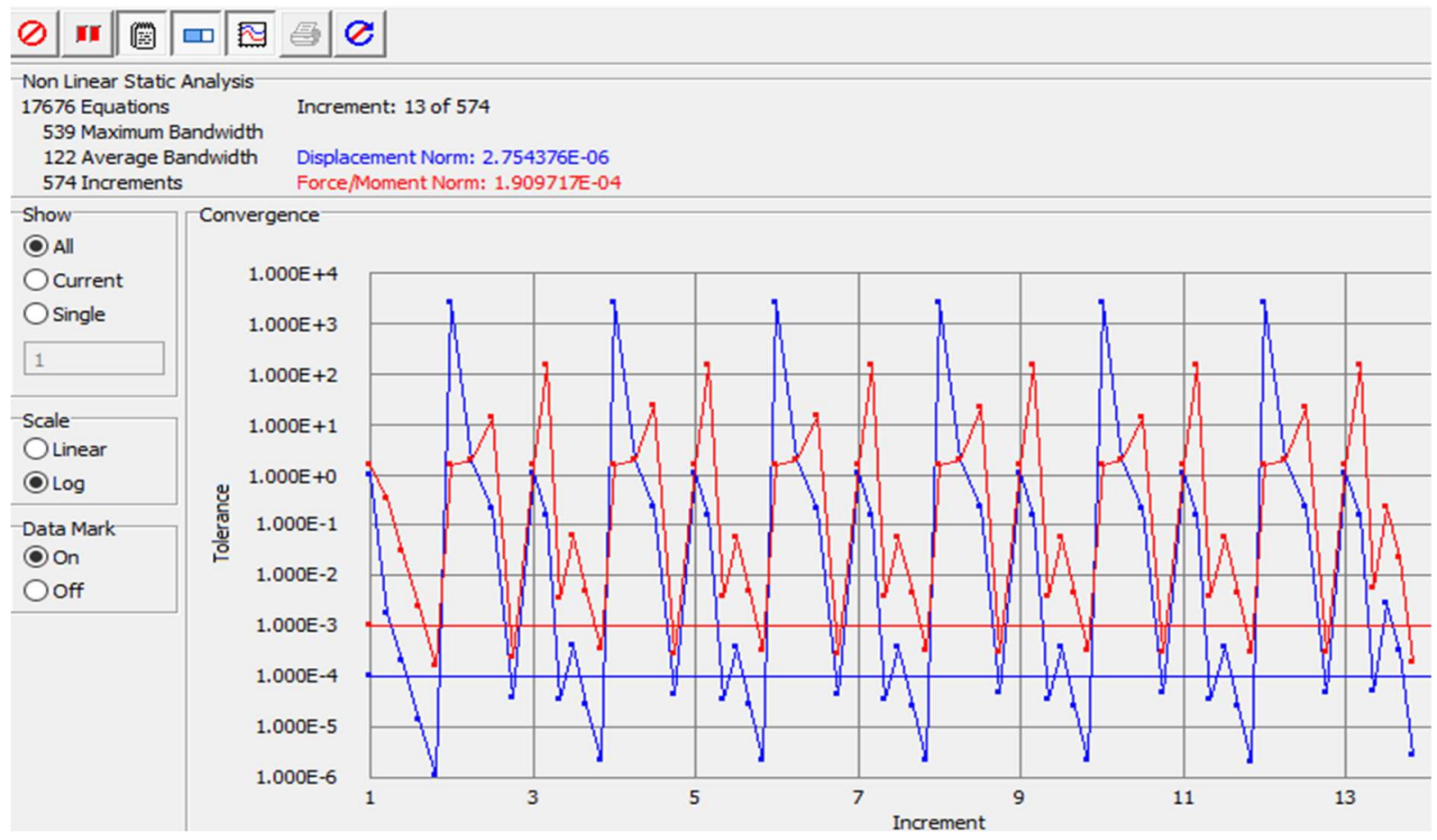

Figure 3.3 Nonlinear Static Solver Convergency Graph (Strand7, 2015)

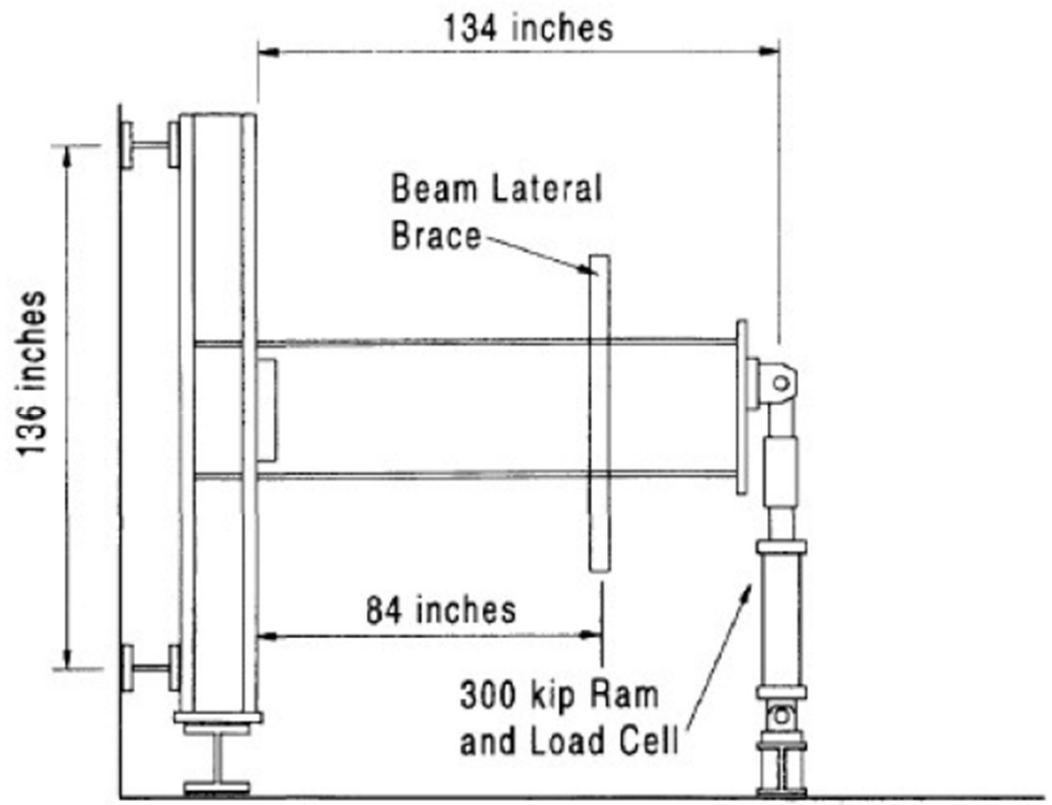


Figure 3.4 RBS Test Setup (Engelhardt et. al, 1998)

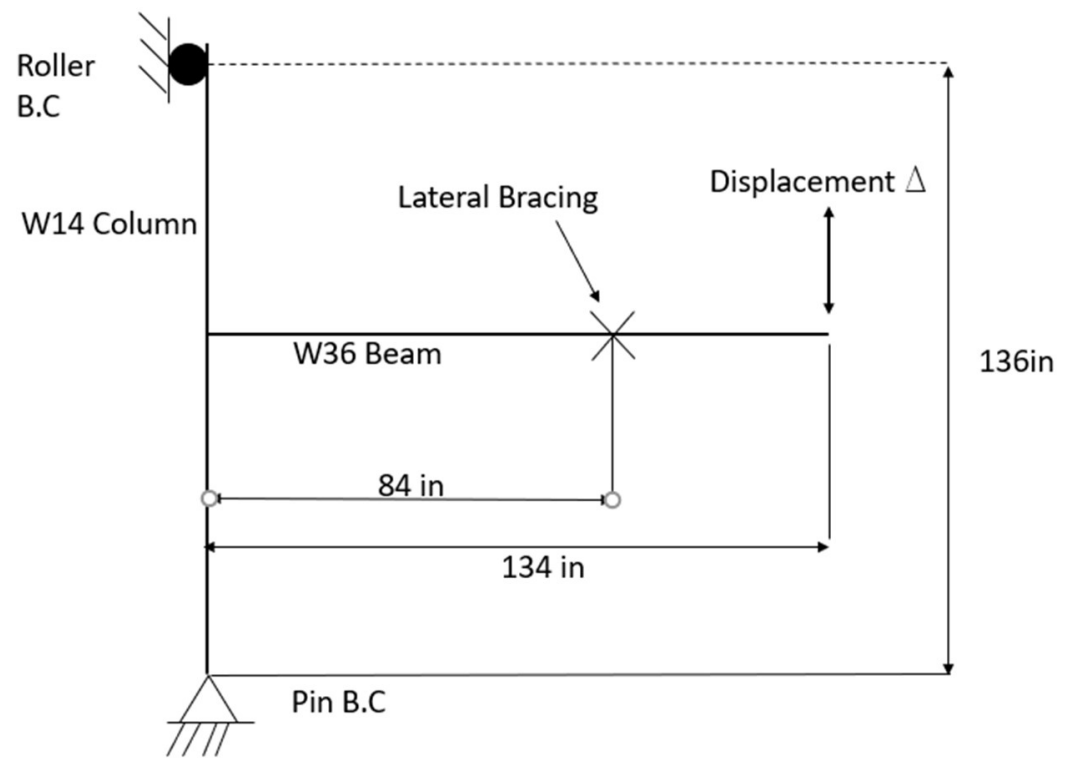

Figure 3.5 Global model span length and boundary condition for FEA

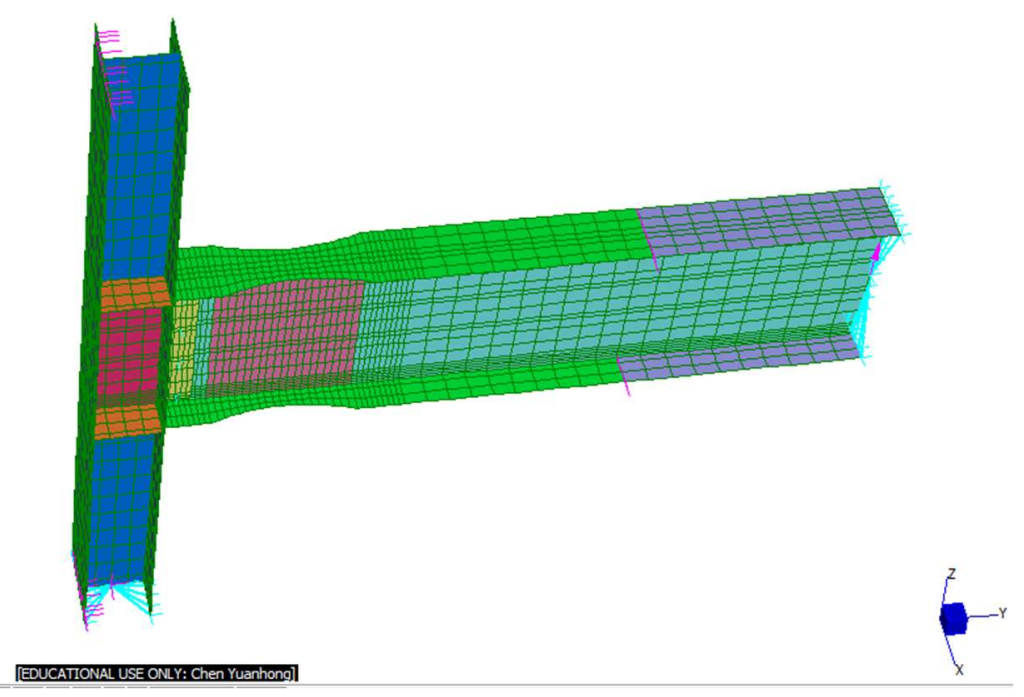

Figure 3.6 3D view of DB2 Plate FEA Model (Strand7, 2016) 


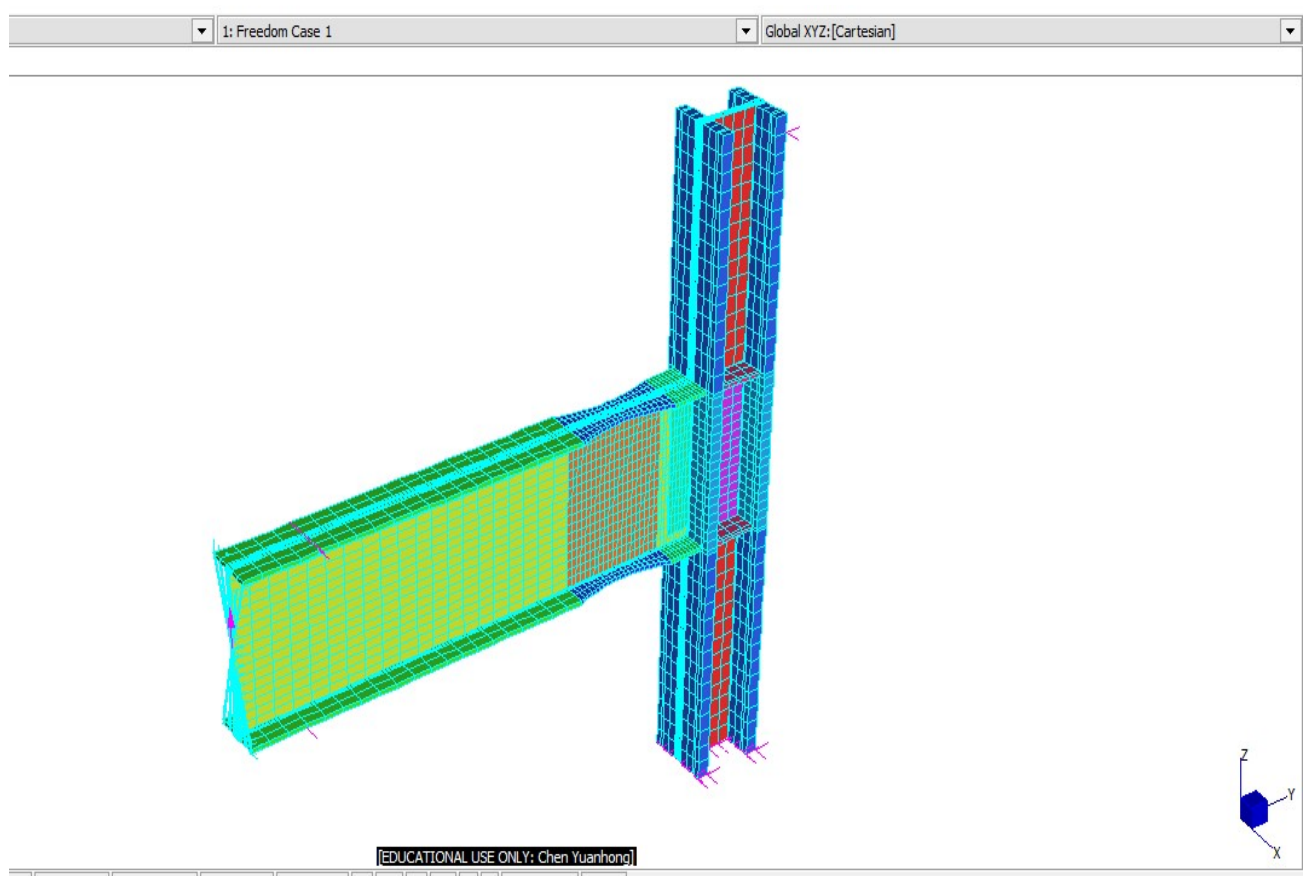

Figure 3.7 DB2 FEA Brick Model 3D View (Strand7, 2016) 


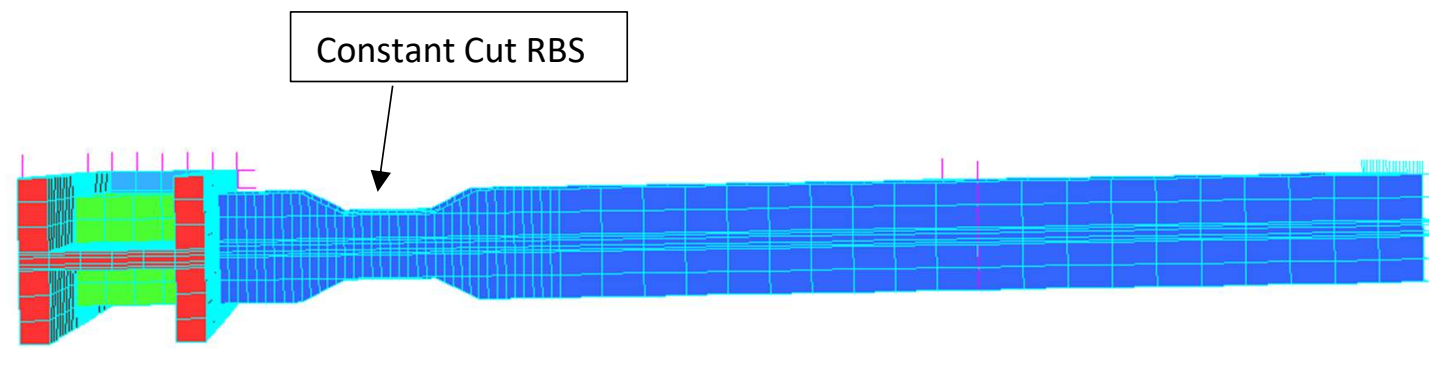

EDUCATIONAL USE ONLY: Chen Yuarhonol

Figure 3.8 Plane View of DB1Brick Model- Constant Cut RBS

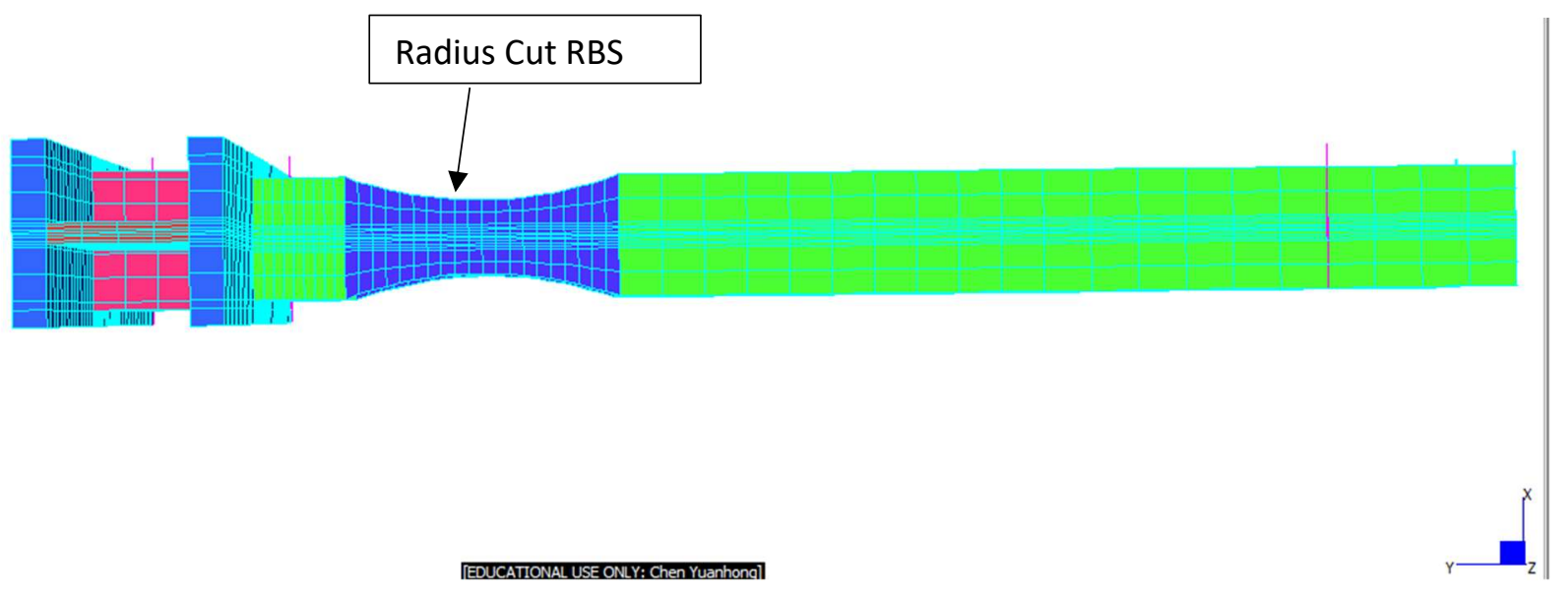

Figure 3.9 Plan View of DB2Brick Model-Radius Cut RBS 

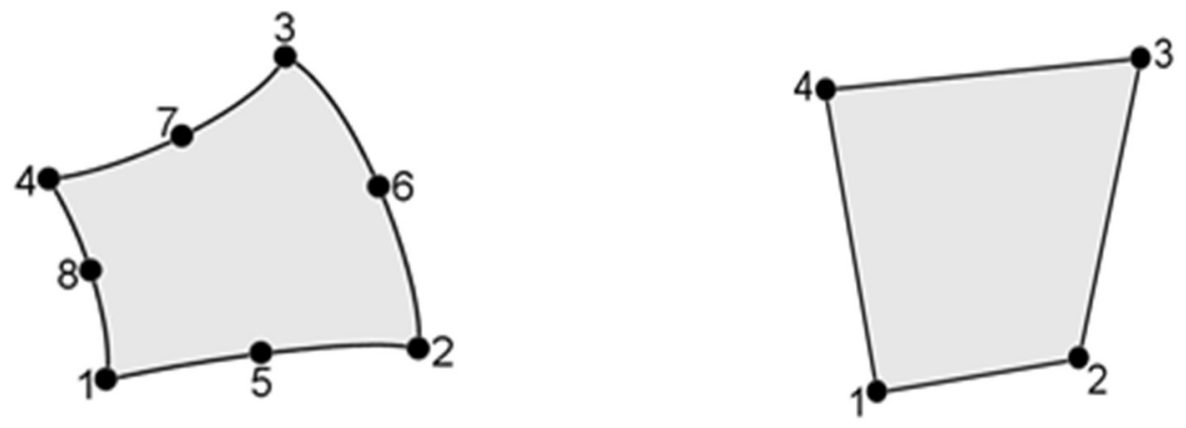

Figure 3.10 Plate Elements for RBS FEA Modeling (left ) Quad8: 8-node quadrilateral element.

(right) Quad4: 4-node quadrilateral element

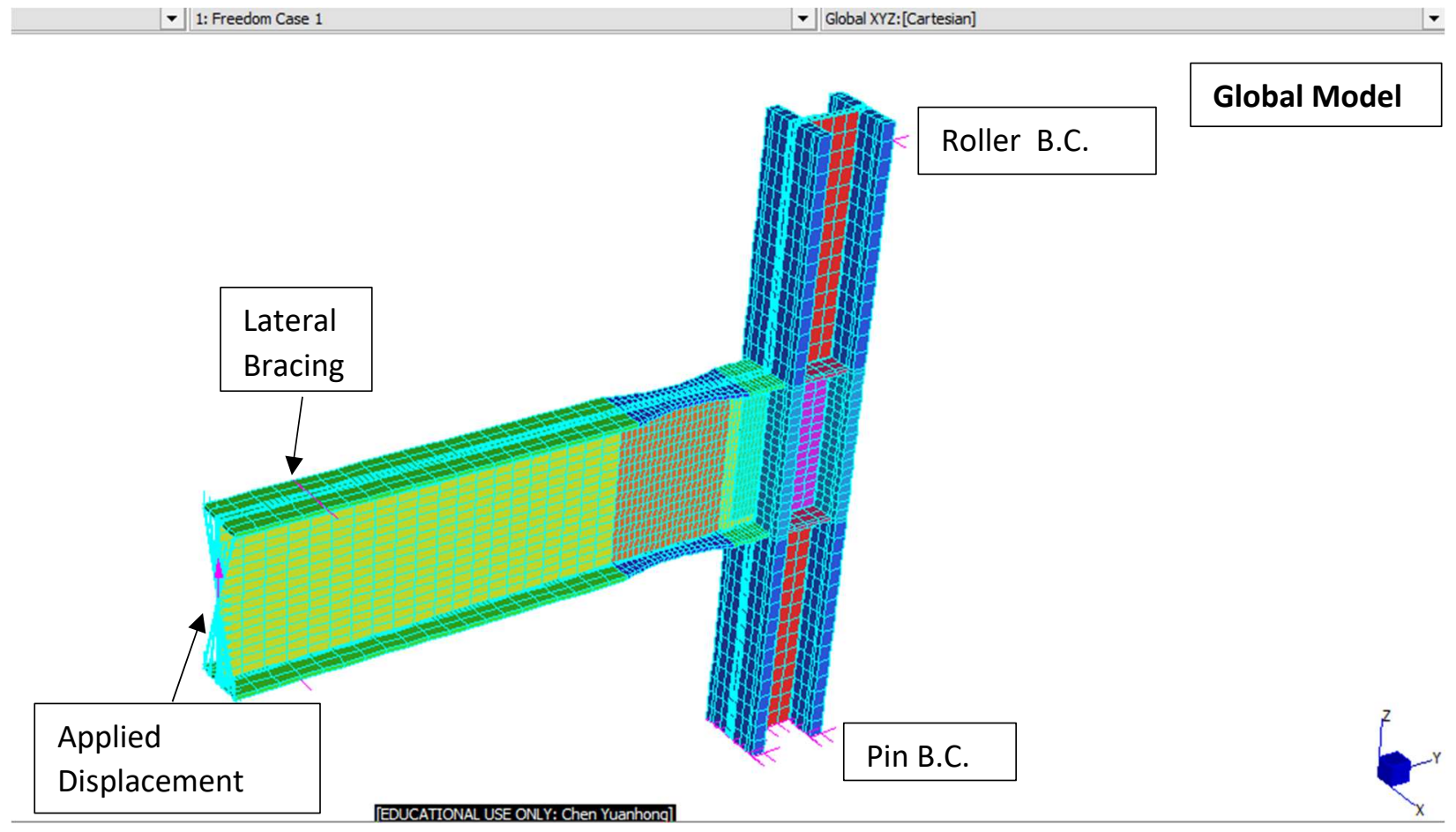

Figure 3.11 FEA Model's Boundary Conditions and Applied Displacement Load 


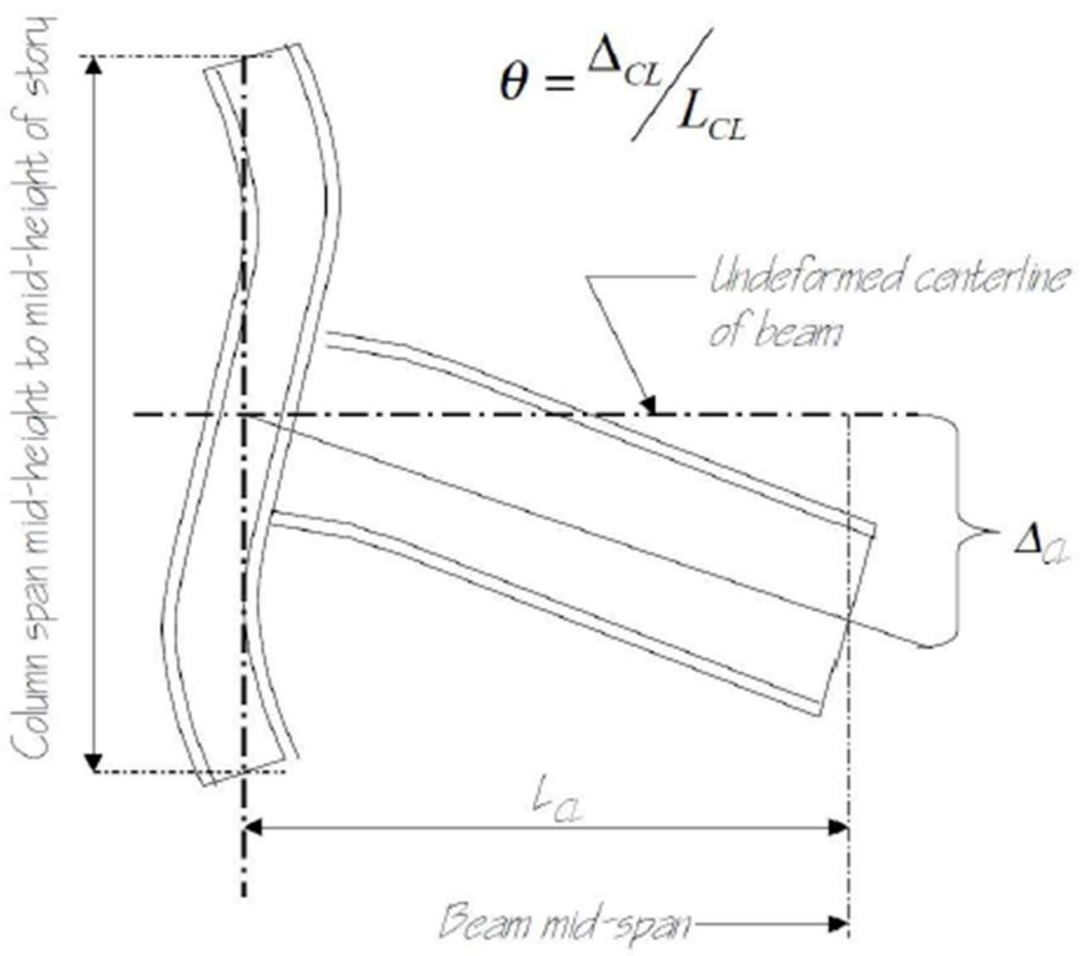

Figure 3.12 Deformed Shape of the RBS Connection During Cyclic Testing (FEMA 350)

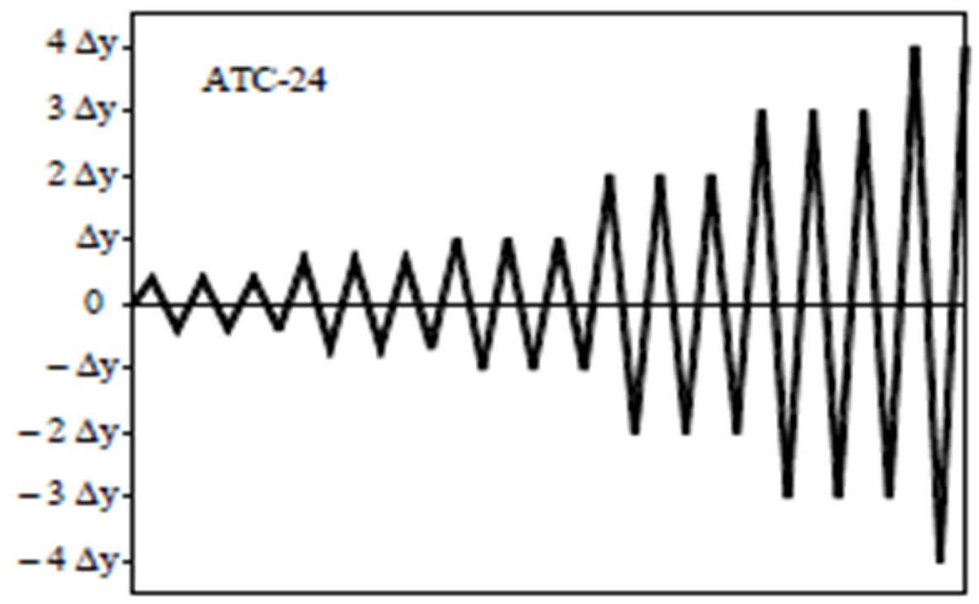

Figure 3.13 ATC-24 Loading Protocol with Displacement Loading 


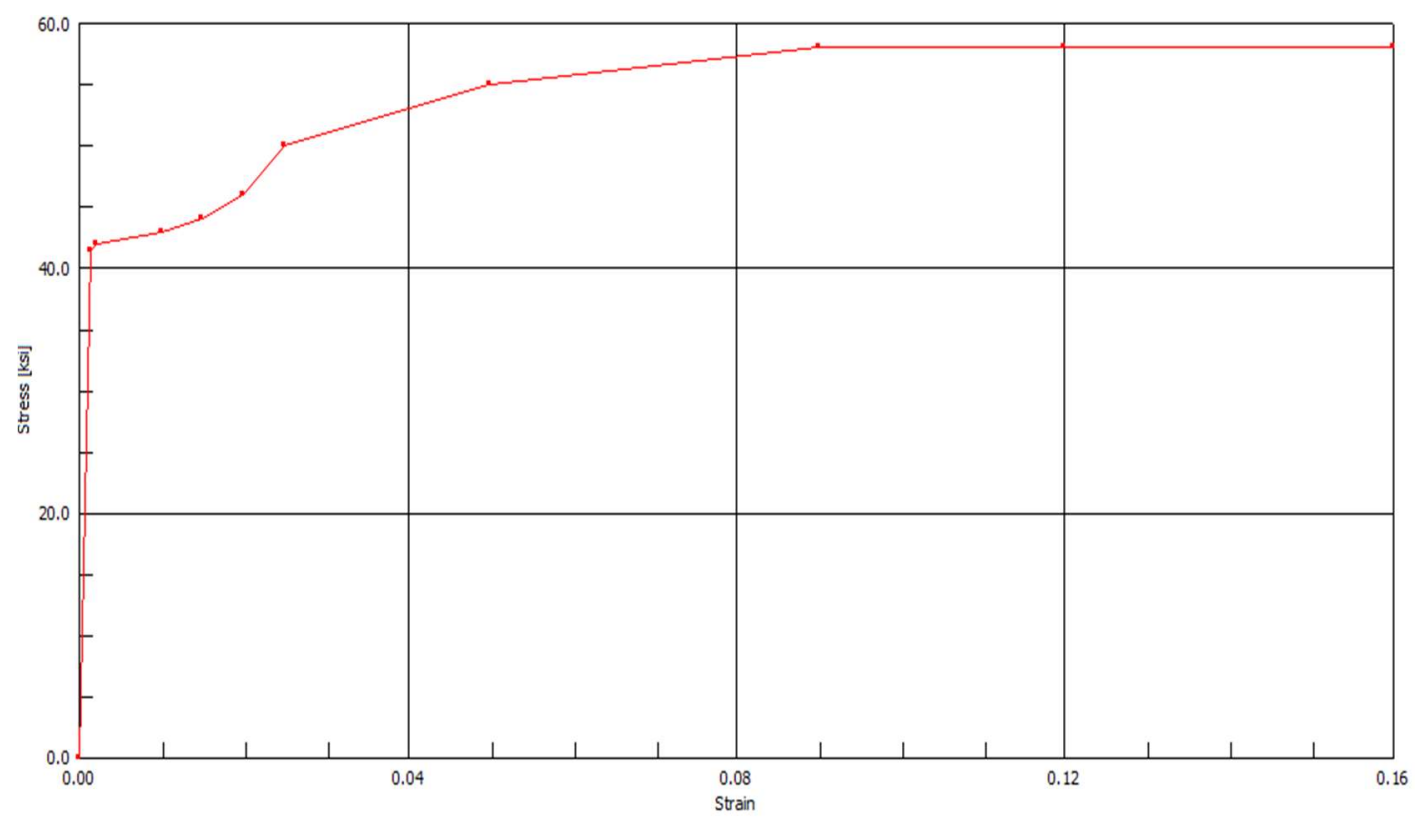

Figure 3.14 Modified Stress vs Strain Curve for Beam Flange

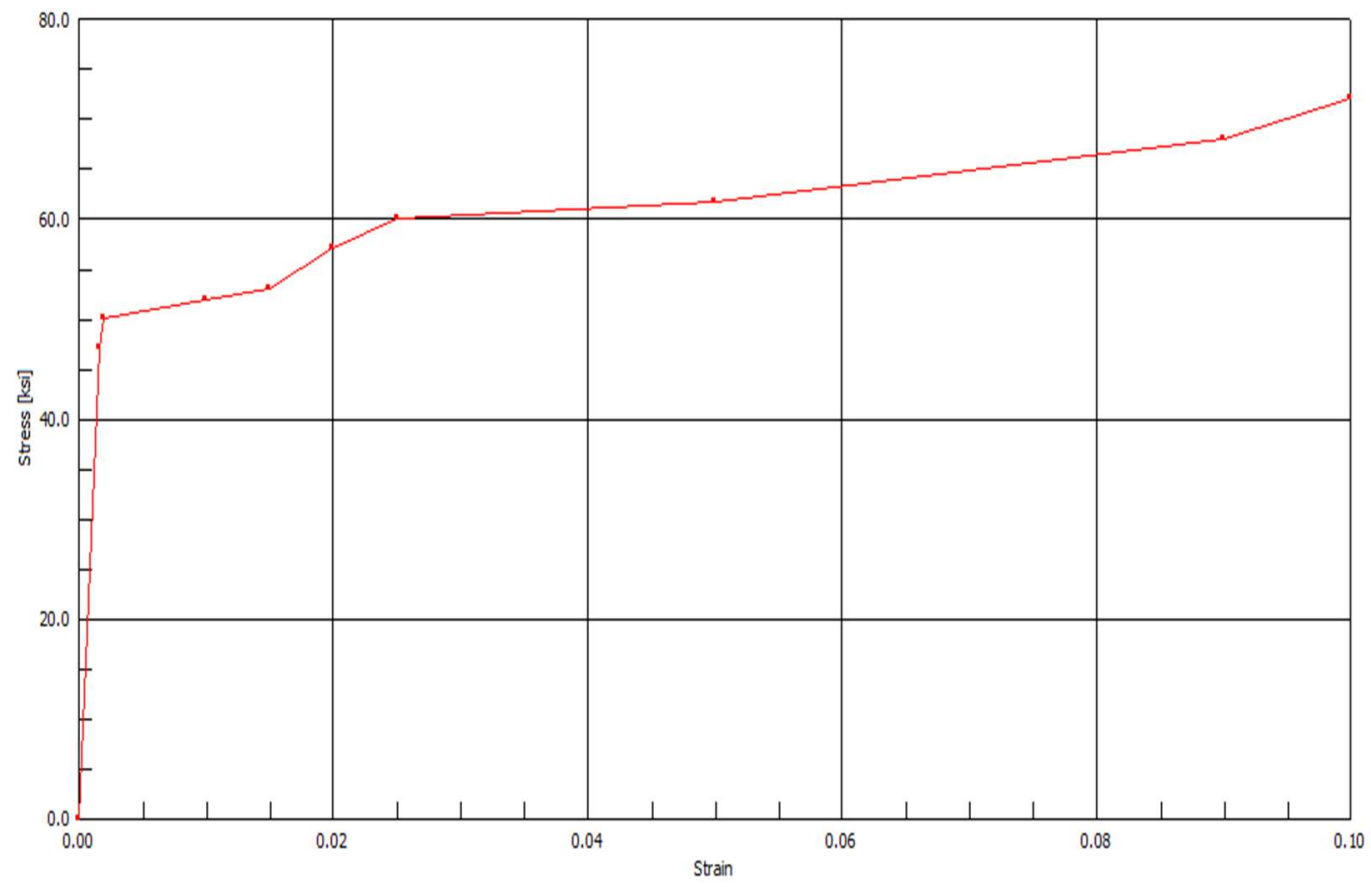

Figure 3.15 Modified Stress vs Strain Curve for Beam Web 


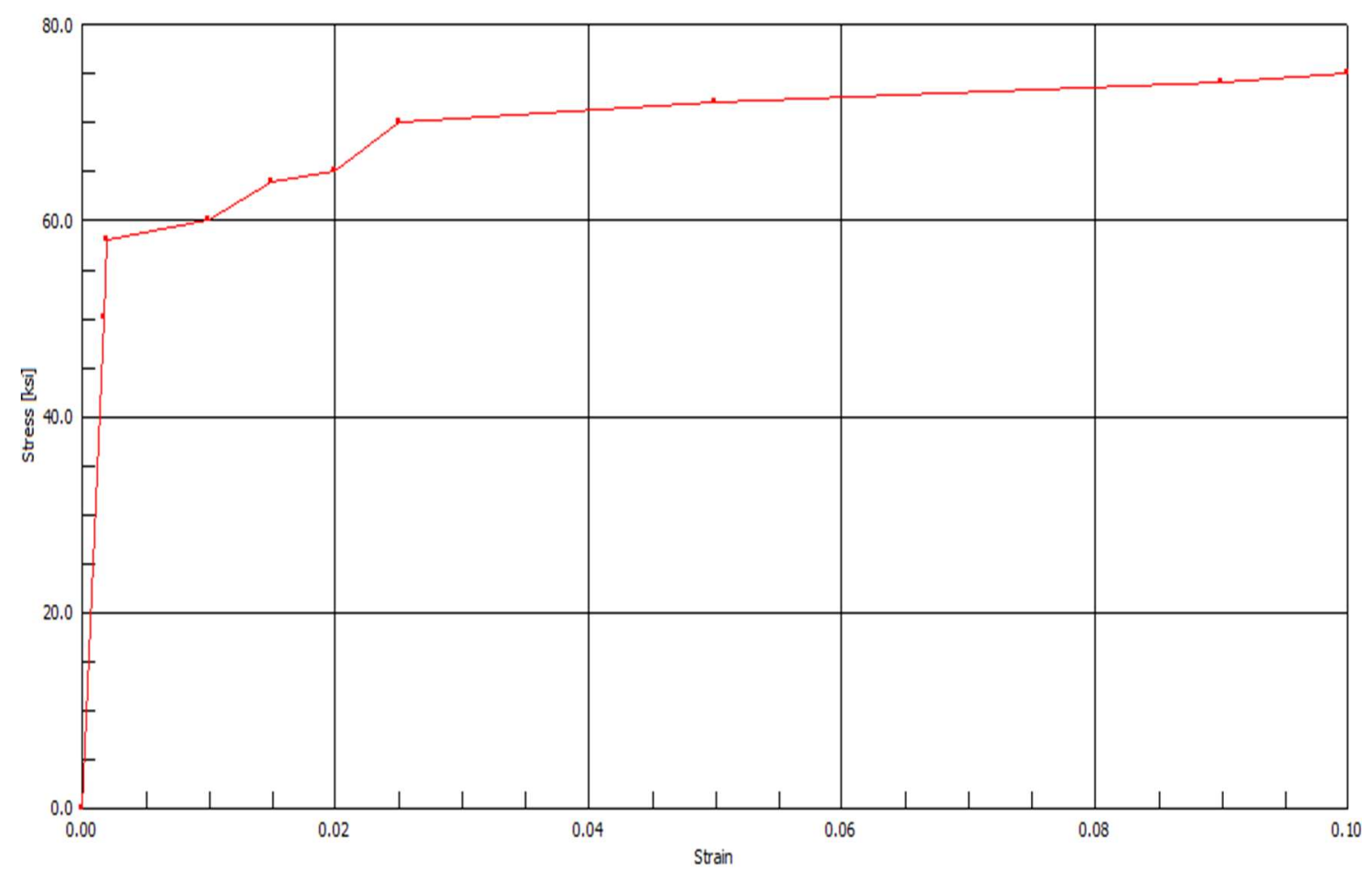

Figure 3.16 Modified Stress vs Strain for Column Flange

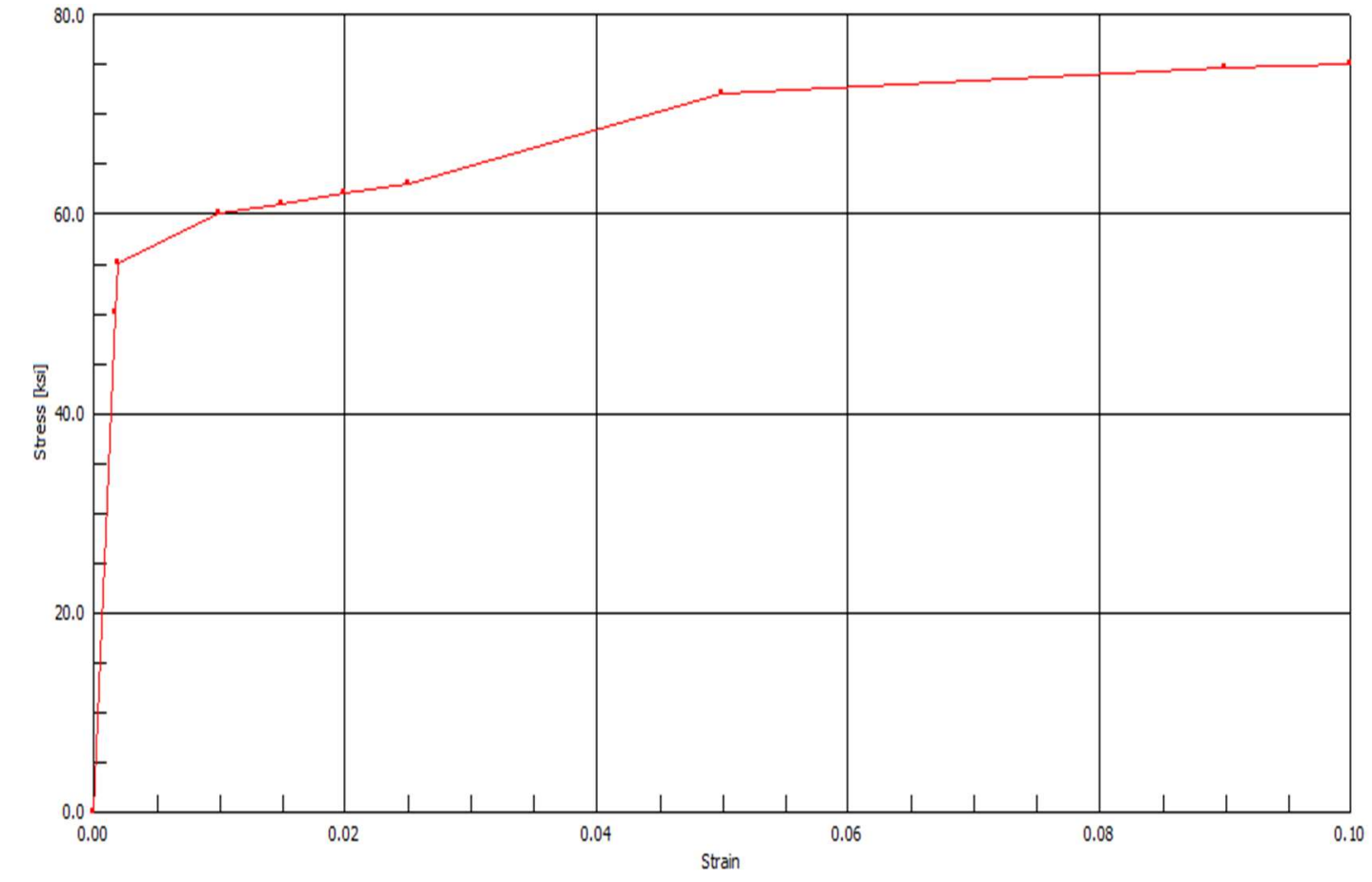

Figure 3.17 Modified Stress vs Strain Curve for Column Web 


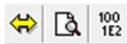

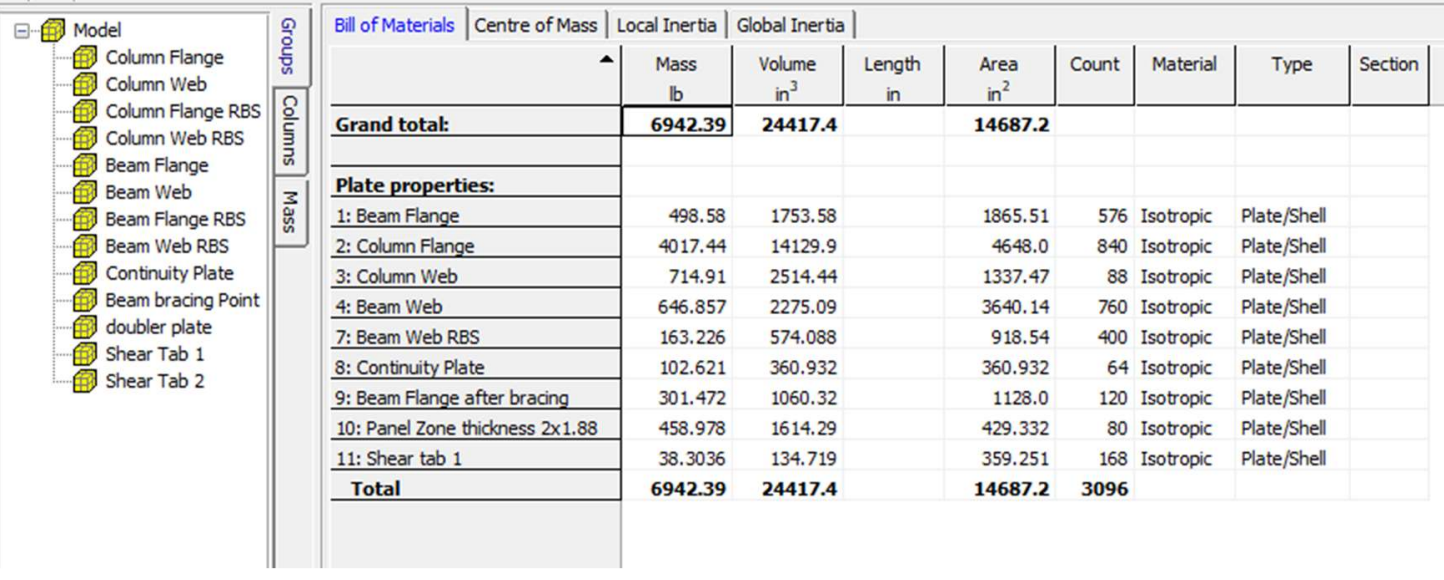

Figure 3.18 Bill of Material of Plate DB2 Model

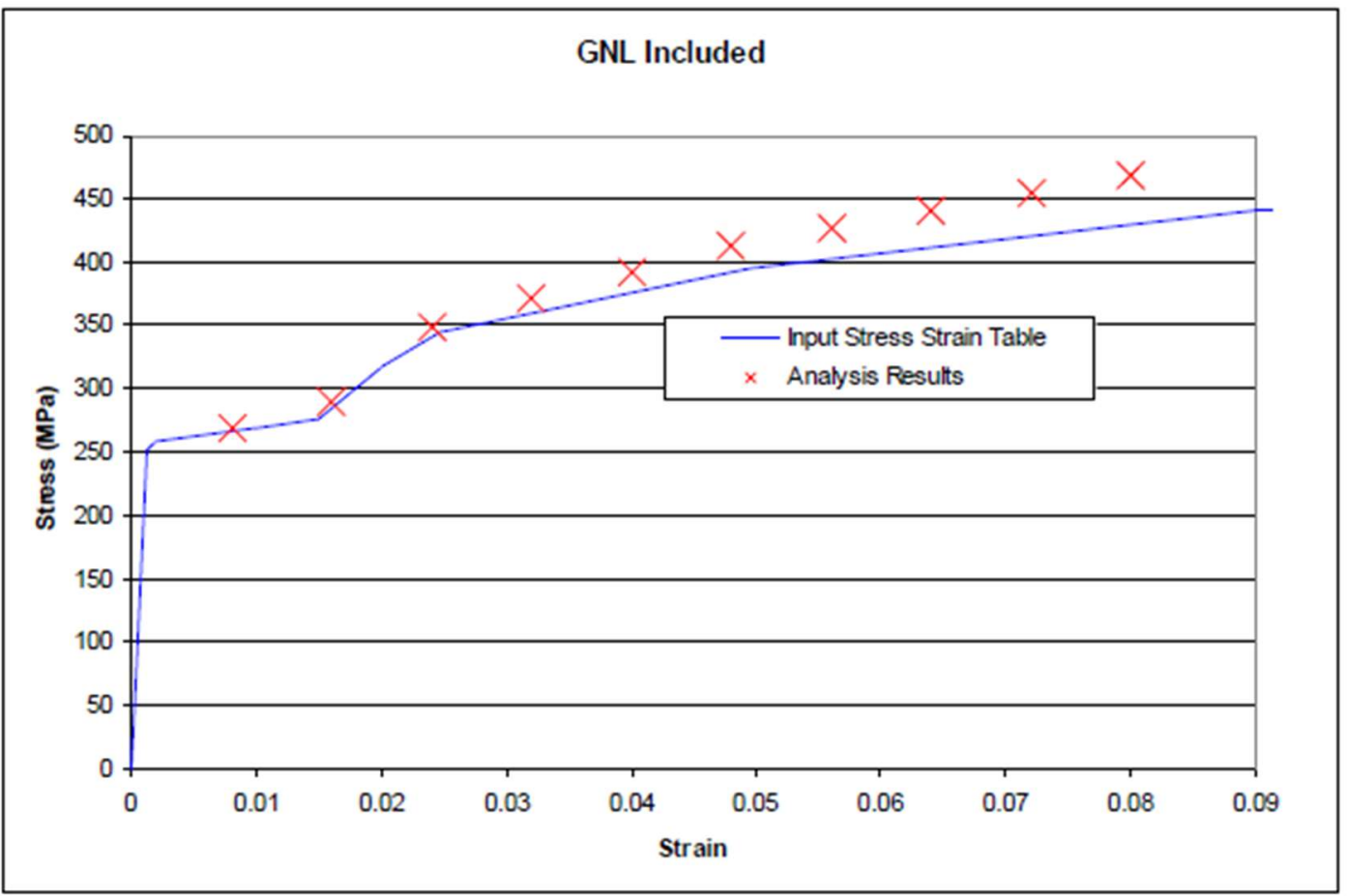

Figure 3.19 Discrepancy Between Analysis Results and Input Stress-Strain Curve for

Typical Mil Steel Material (Strand7 Webnote) 


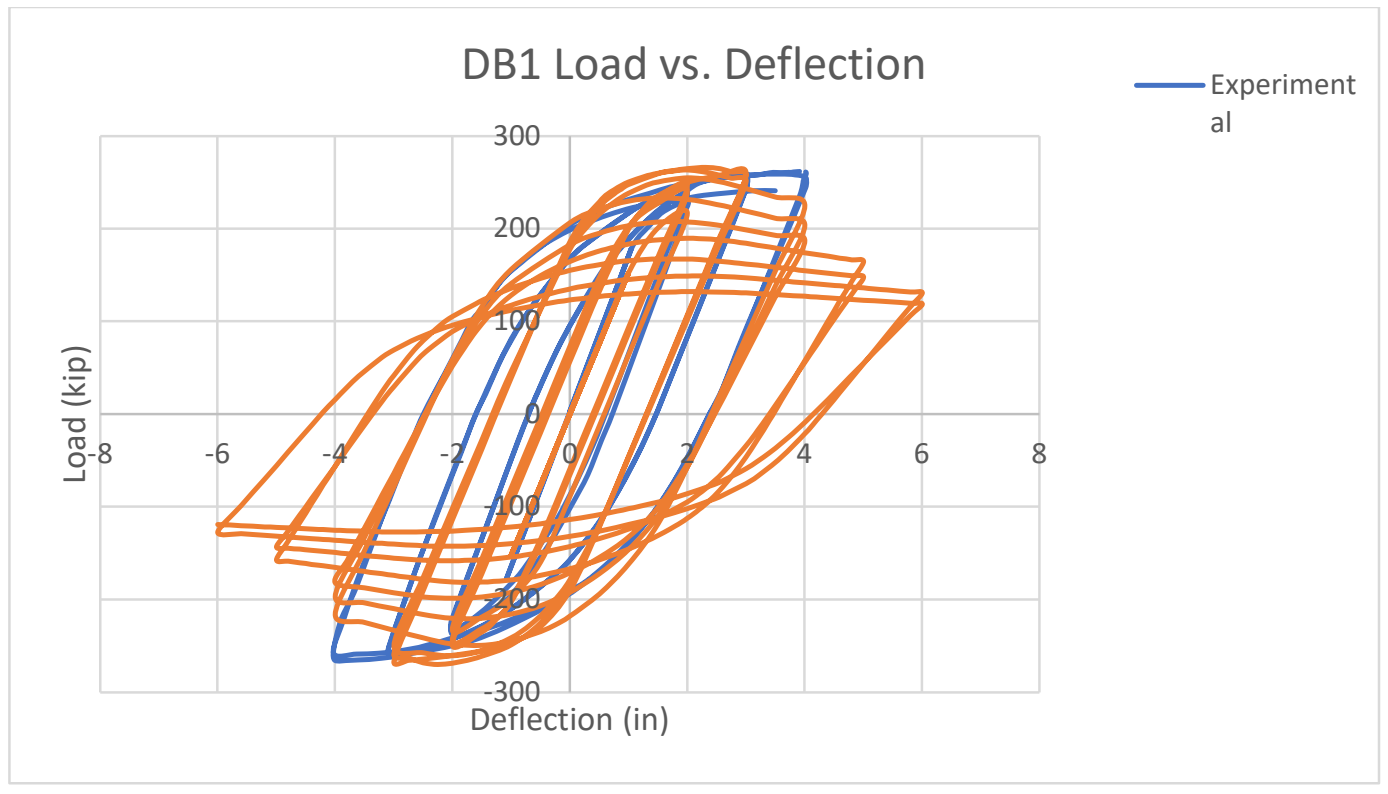

Figure 3.20 DB1 Plate Model Load vs Deflection Plot

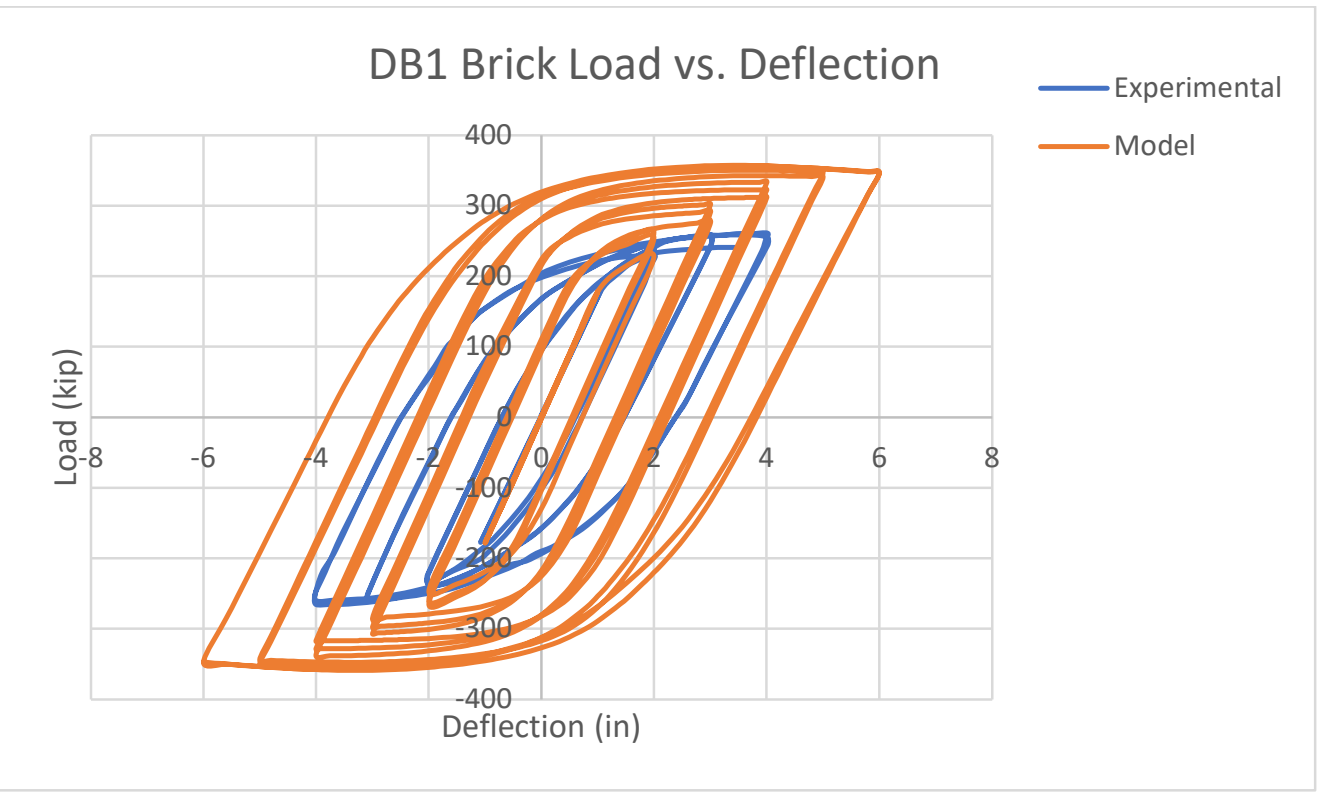

Figure 3.21 DB1 Brick Model Load vs Deflection Plot 

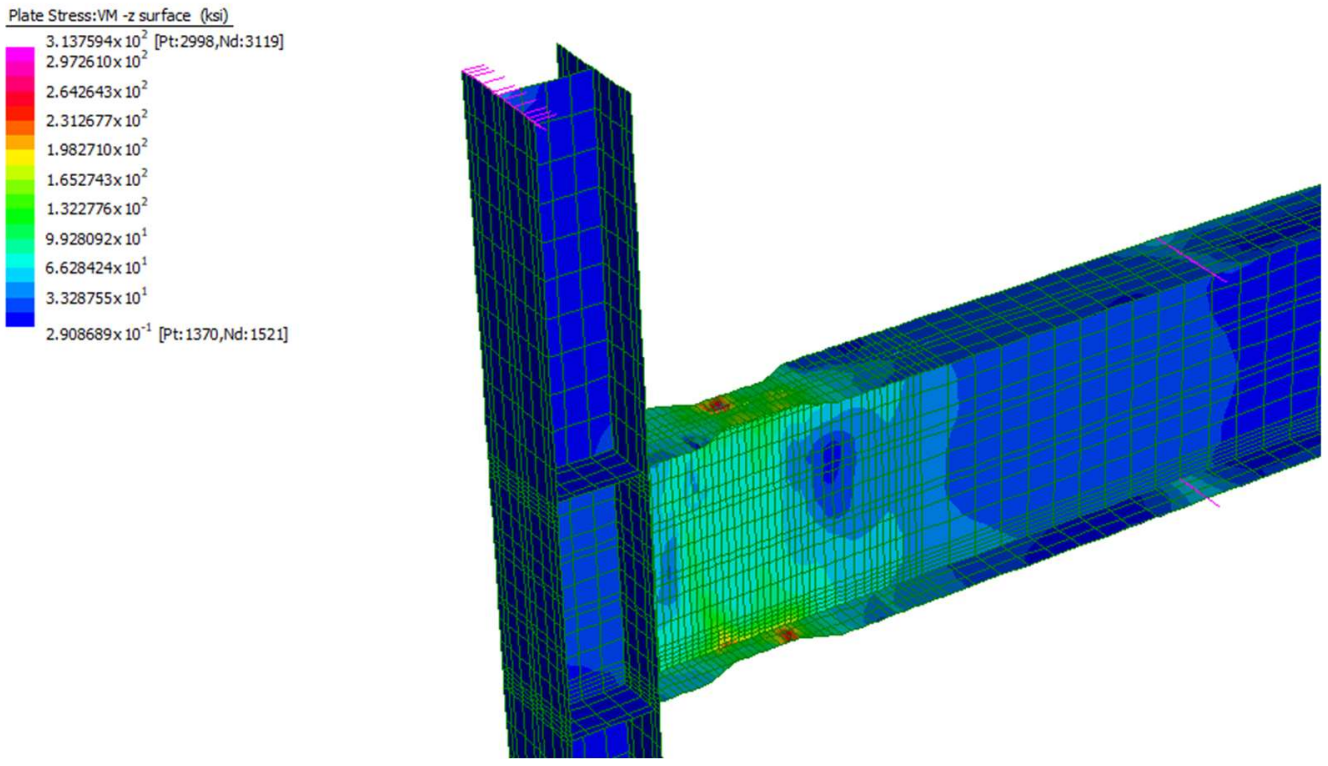

Figure 3.22 Von Mises Stress of DB1 Model at 0.05 radian Rotation ( $0 \%$ displacement scale)
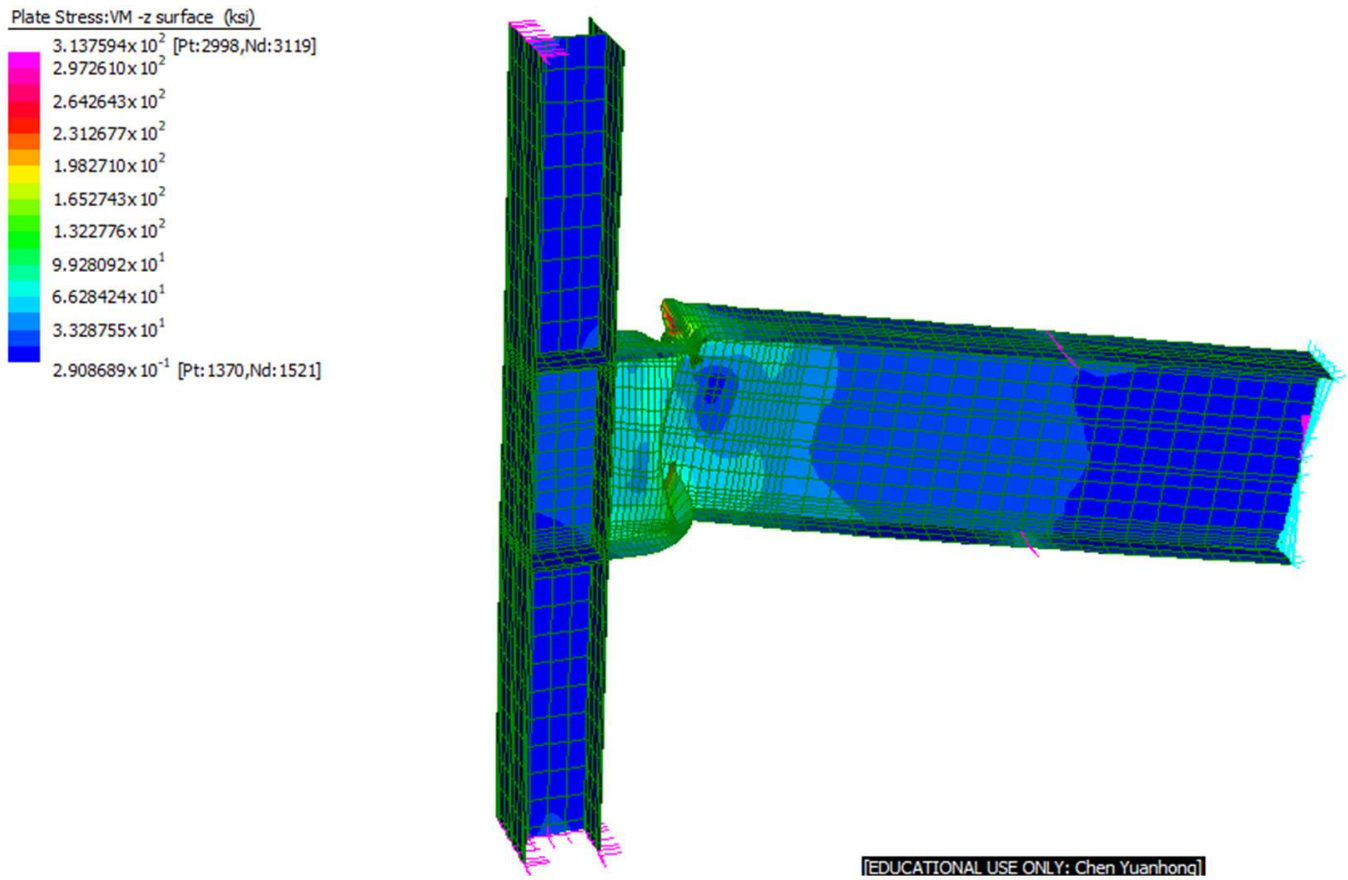

Figure 3.23 Deformed Shape of DB1 Plate Model at 0.05 Radian Rotation (10\% displacement scale) 

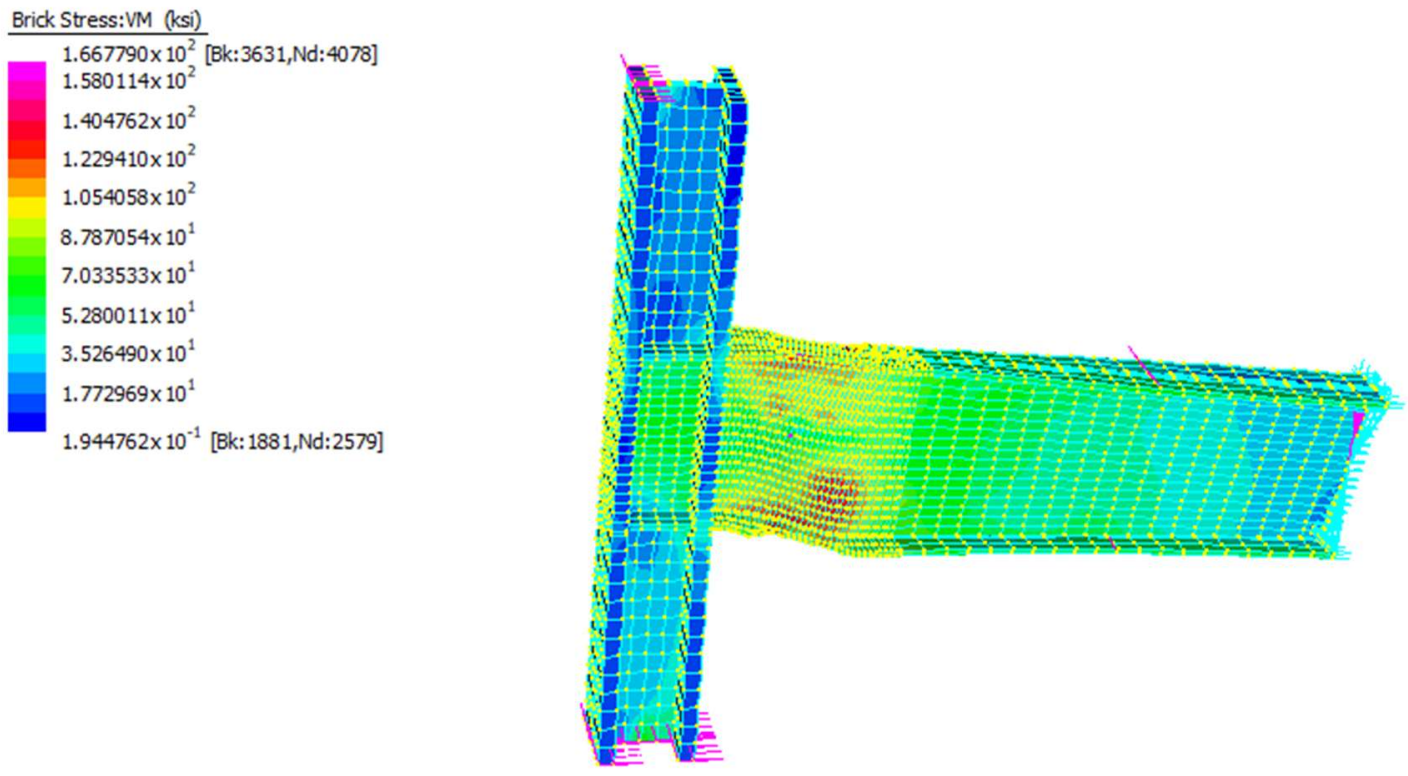

Figure 3.24 Deformed Shape of DB1 Brick Model at 0.05 Radian Rotation

$$
(10 \% \text { displacement scale })
$$

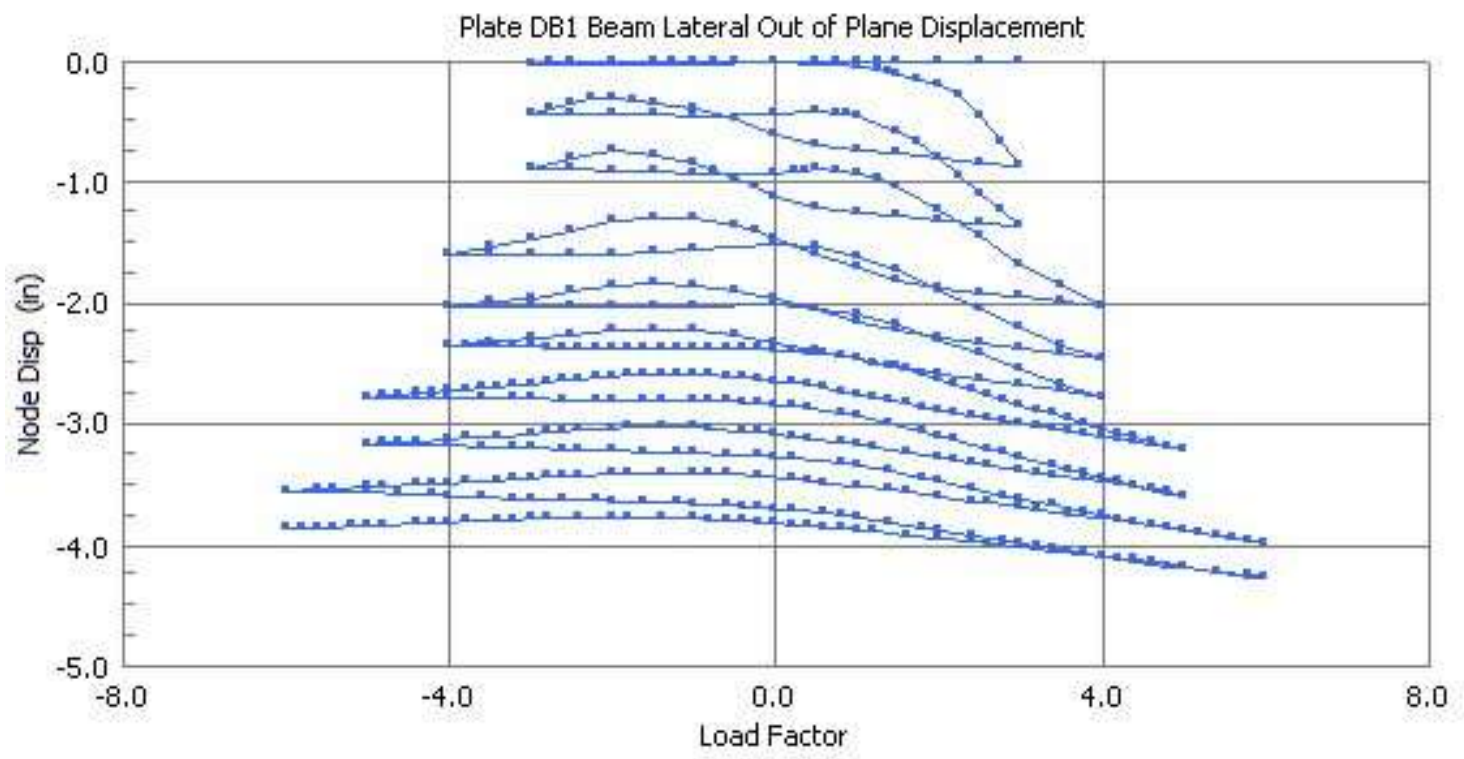

Figure 3.25 Beam Lateral Out of Plane Displacement of DB1 Plate Model 


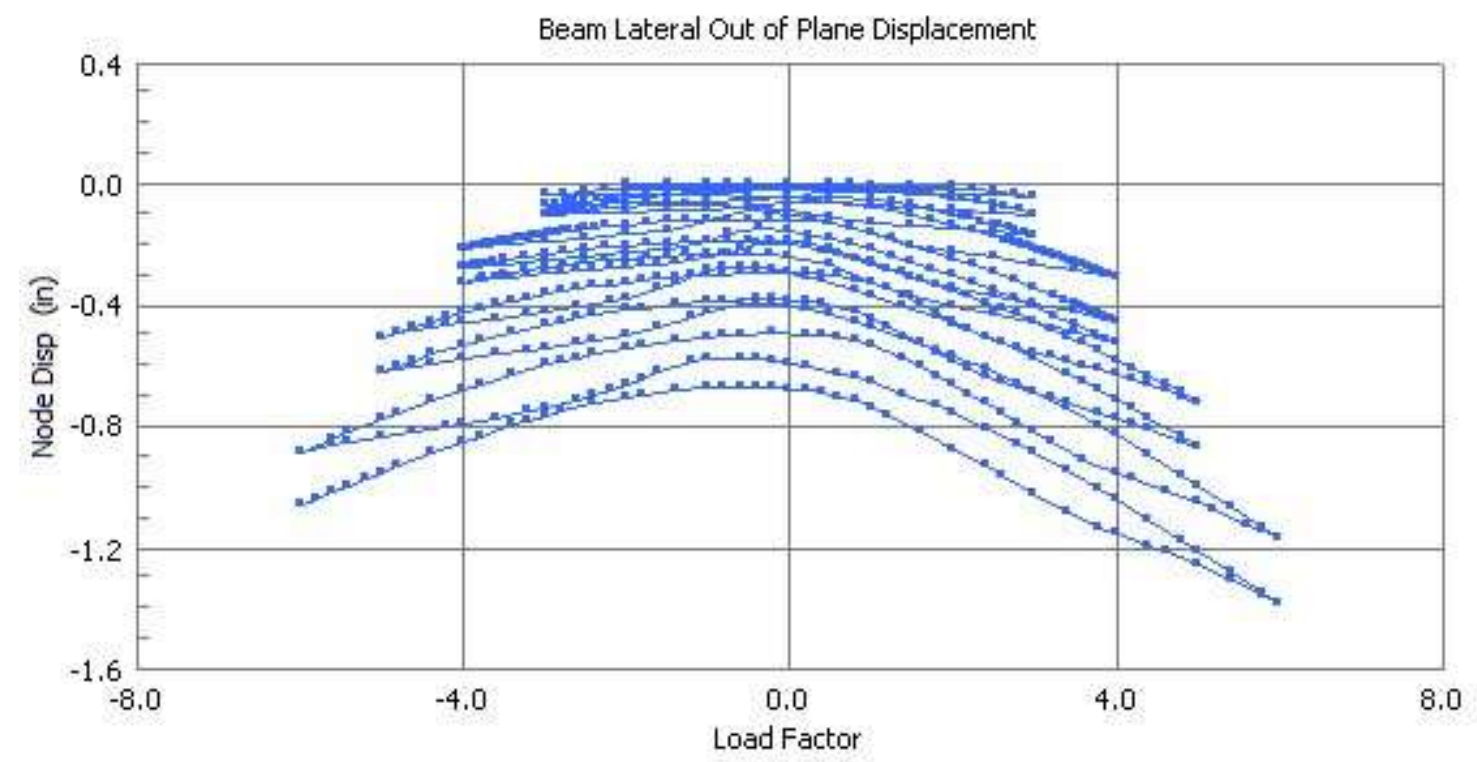

Figure 3.26 Beam Lateral Out of Plane Displacement of DB1 Brick Model
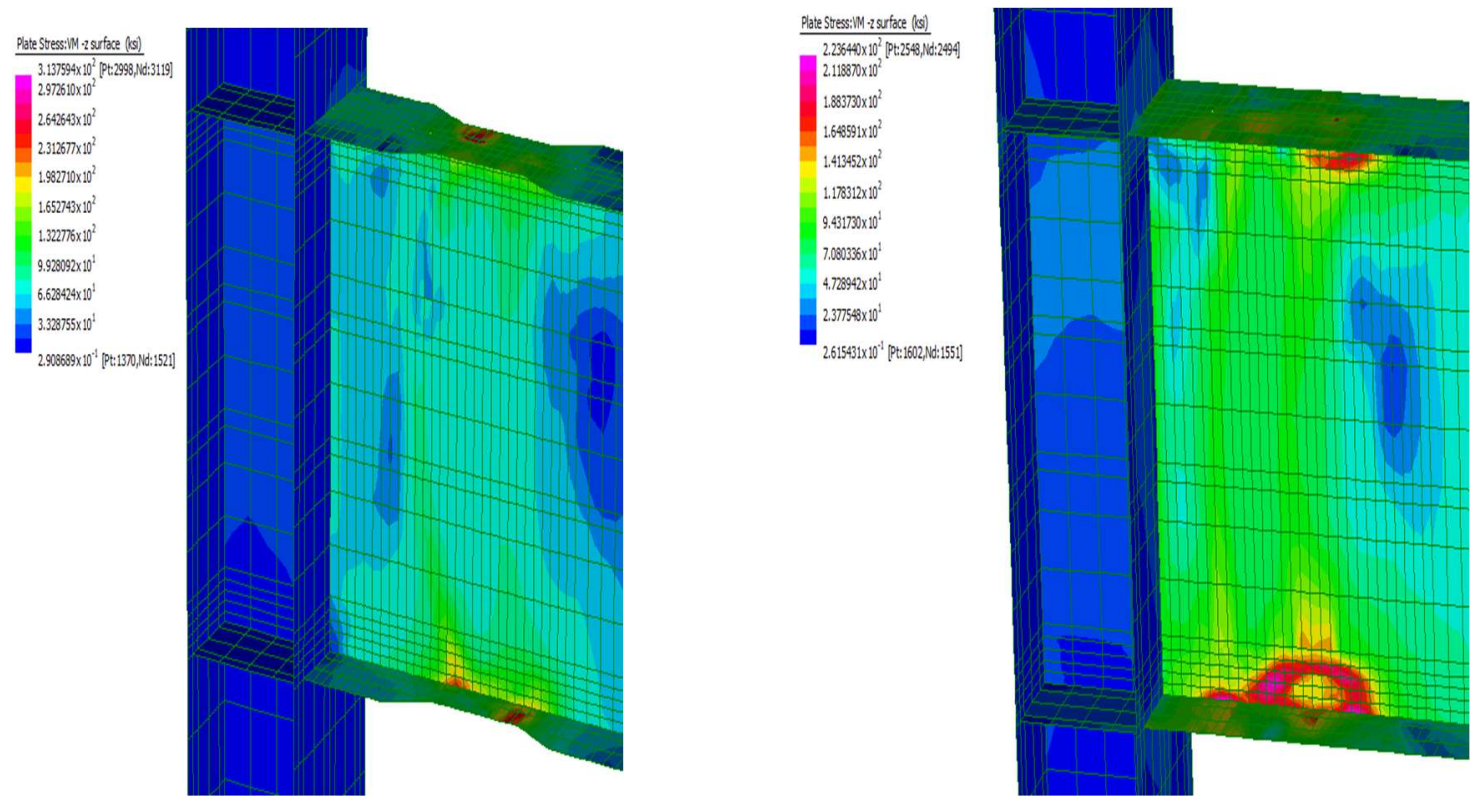

Figure 3.27 Comparison of connection with and without Reduced Beam Section (DB1) 

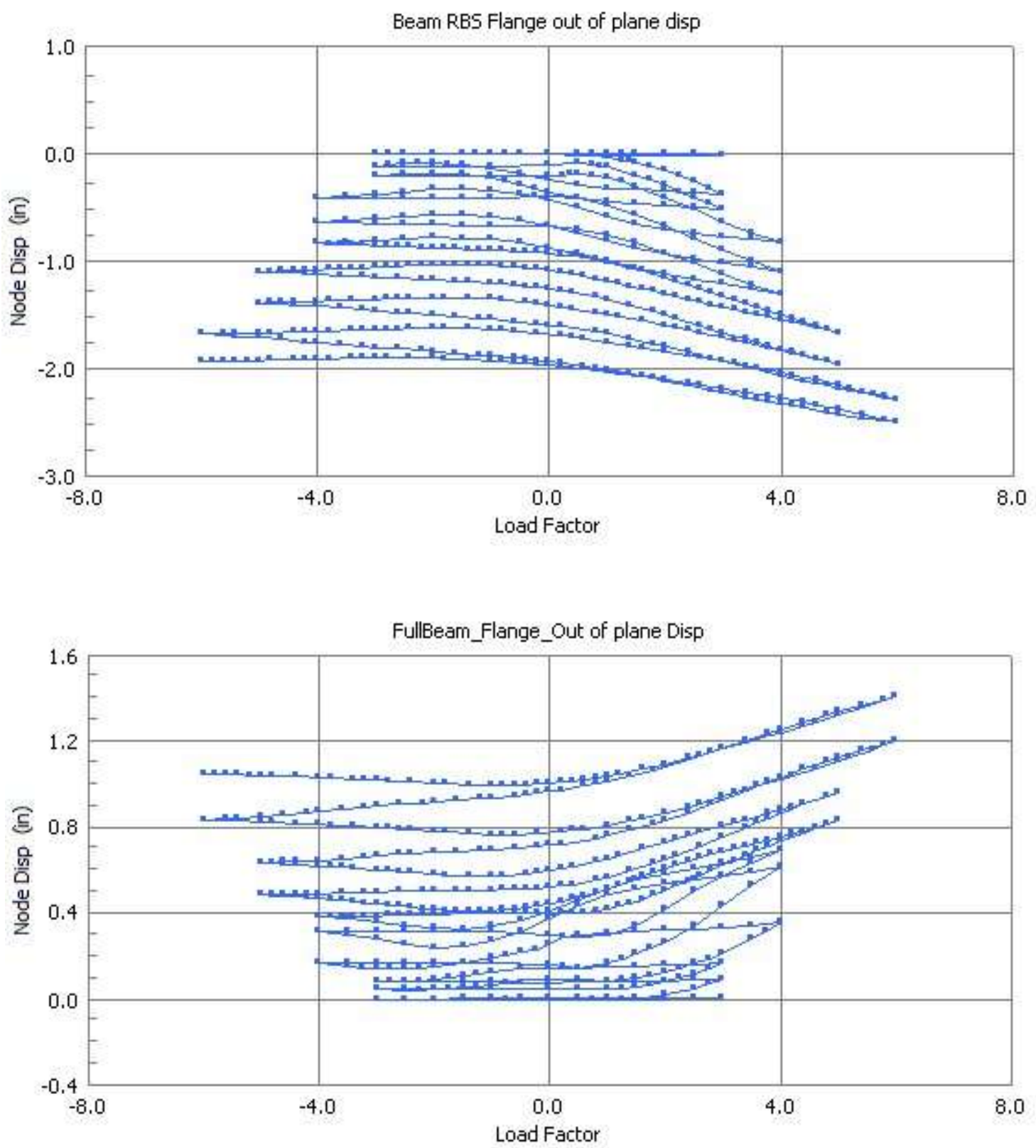

Figure 3.28 DB1 Beam Flange Out of Plane Displacement: RBS Connection (Top), Full Beam Section (Bottom) 

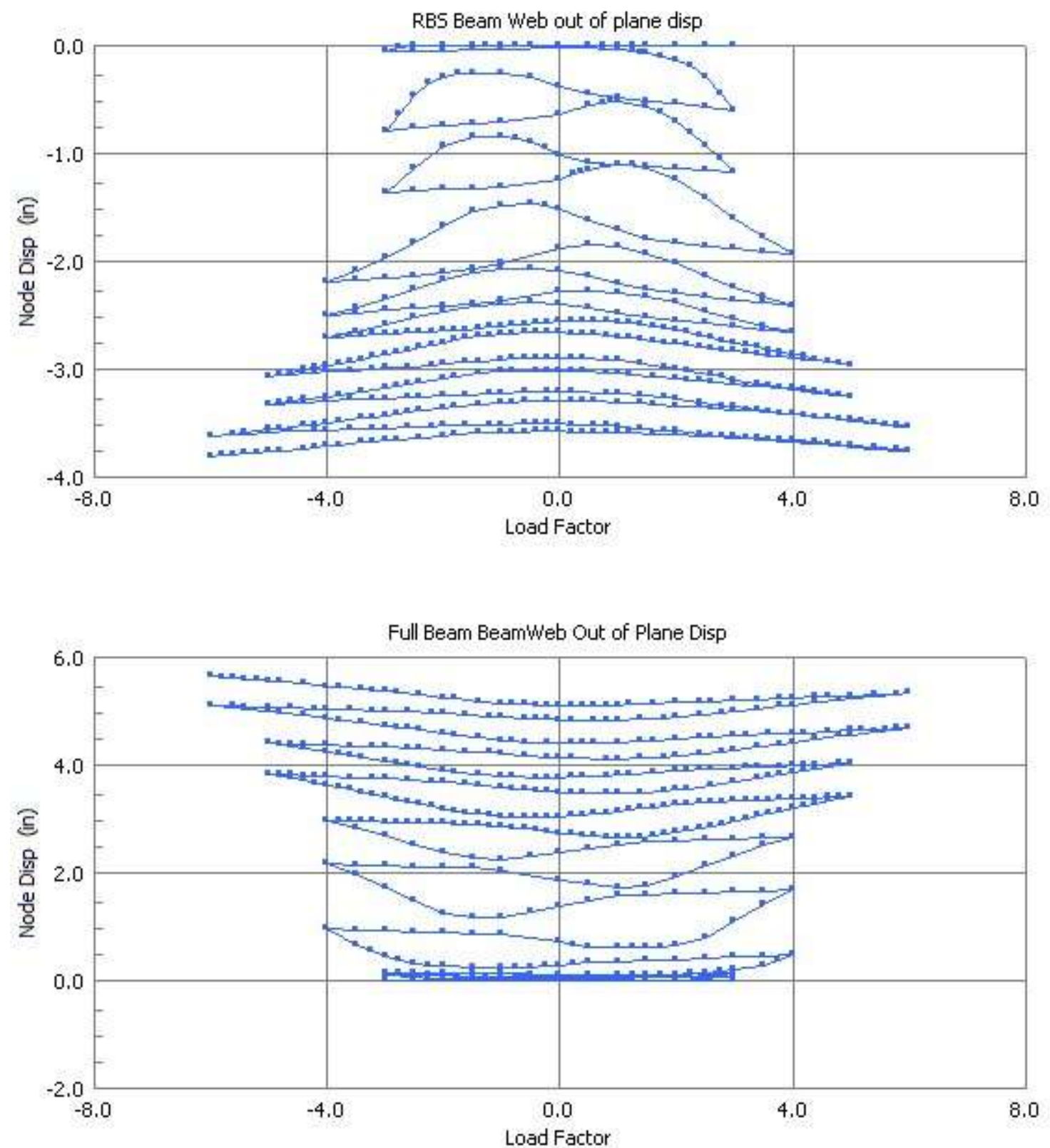

Figure 3.29 DB1 Beam Web Out of Plane Displacement: RBS Connection (Top), Full Beam Section (Bottom) 


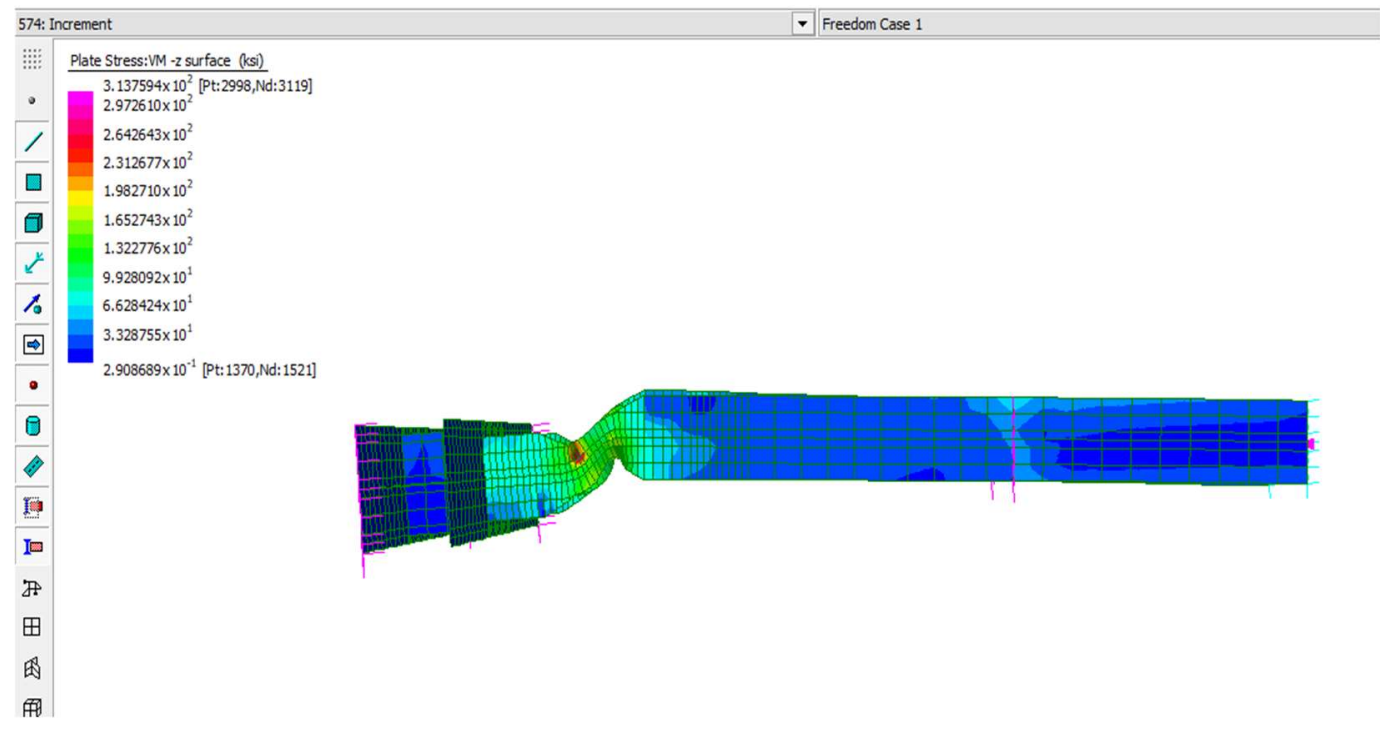

Figure 3.30 Lateral Torsional Buckling of DB1

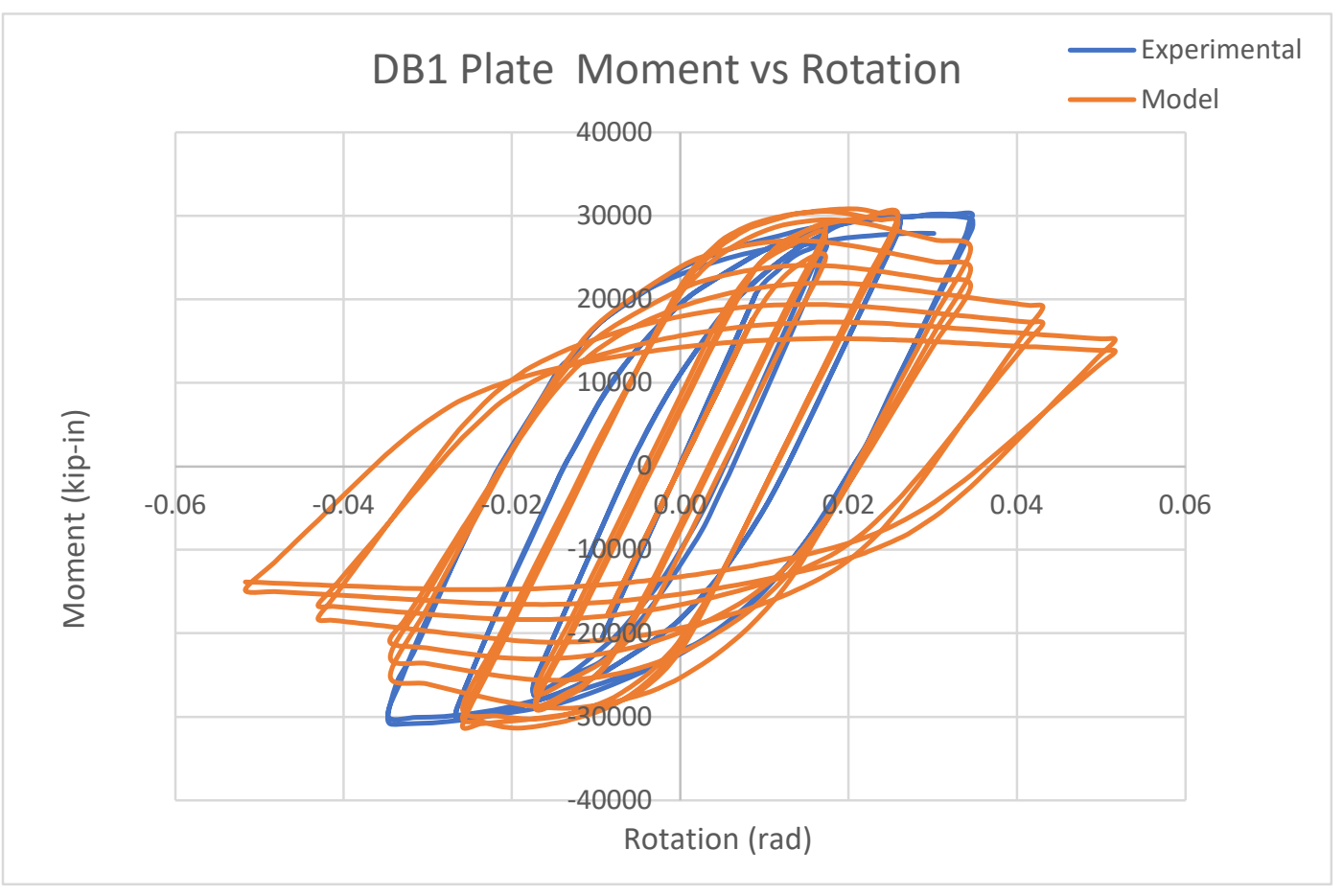

Figure 3.31 DB1 Plate Model Moment vs Rotation Plot 


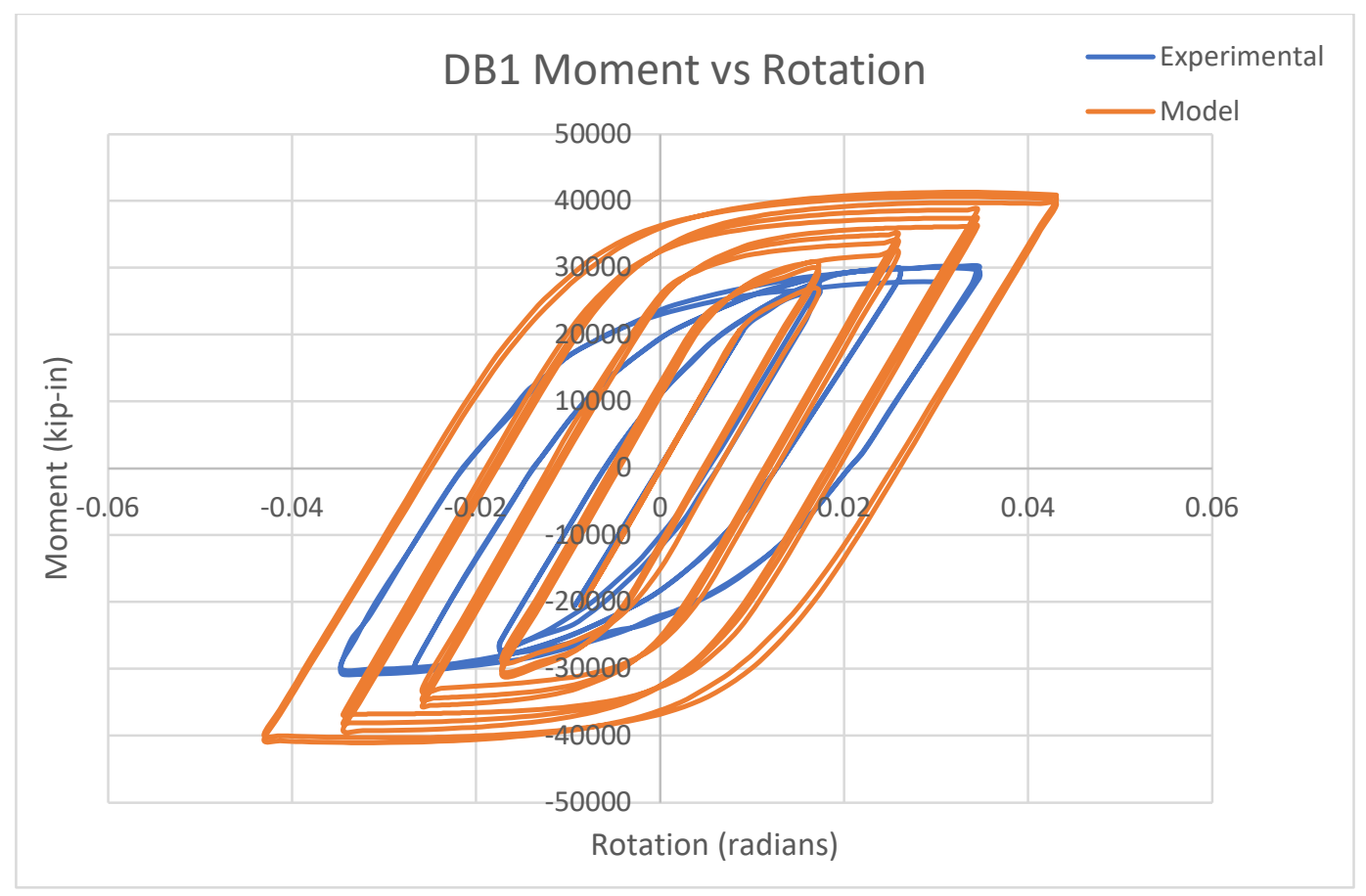

Figure 3.32 DB1 Brick Model Moment vs Rotation Plot

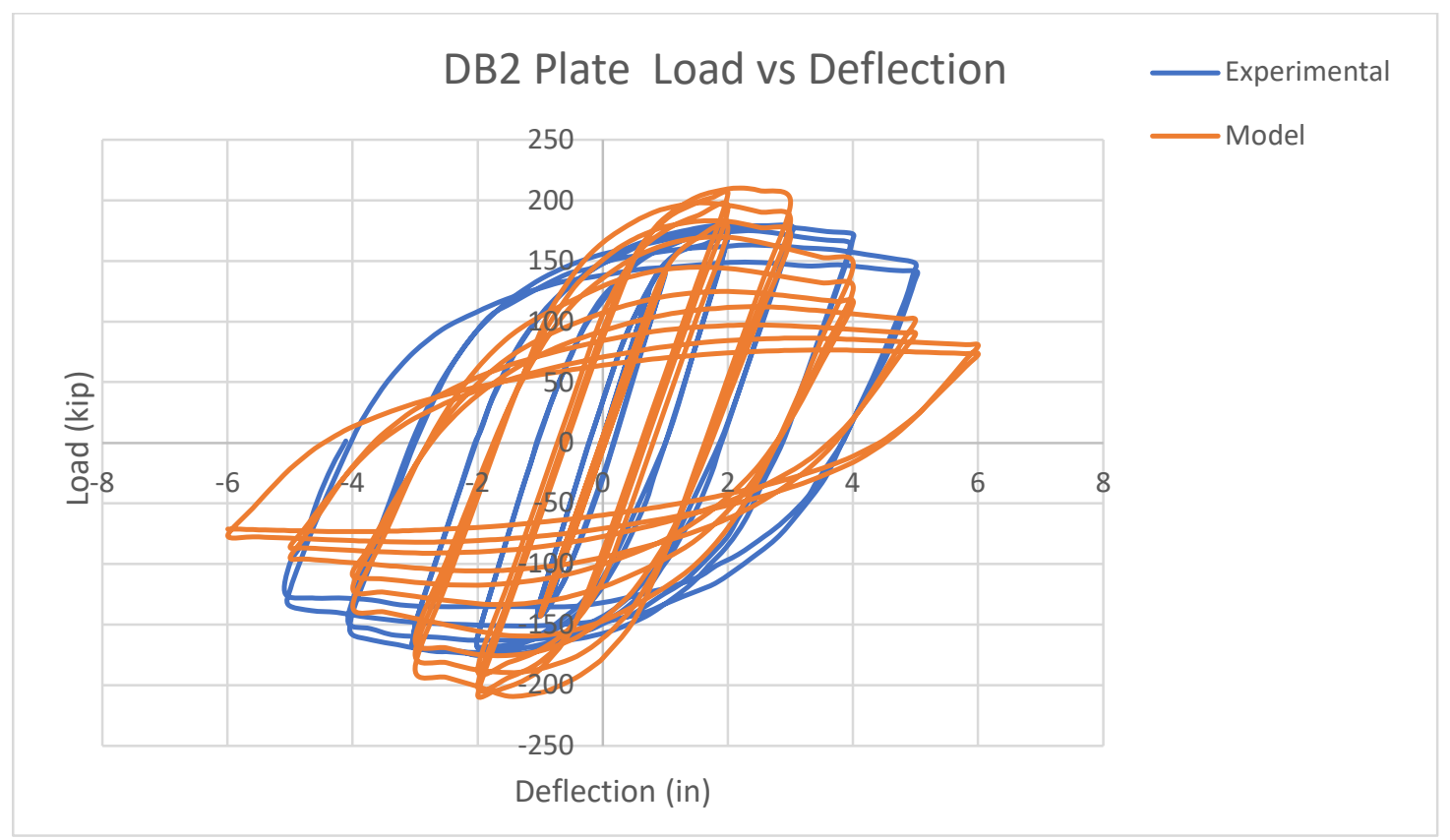

Figure 3.33 DB2 Plate Model Load vs. Deflection Plot 


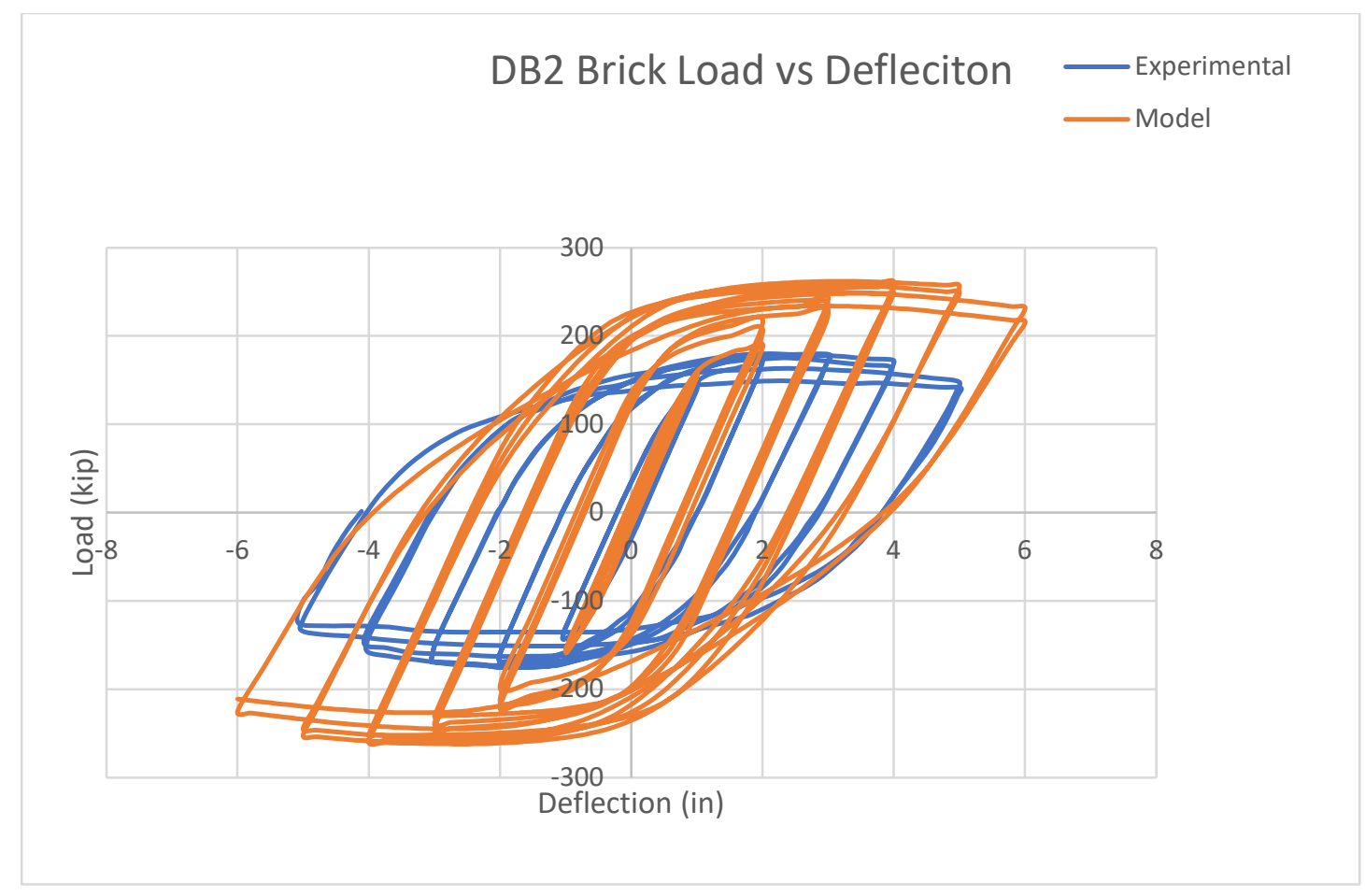

Figure 3.34 DB2 Brick Model Load vs. Deflection Plot

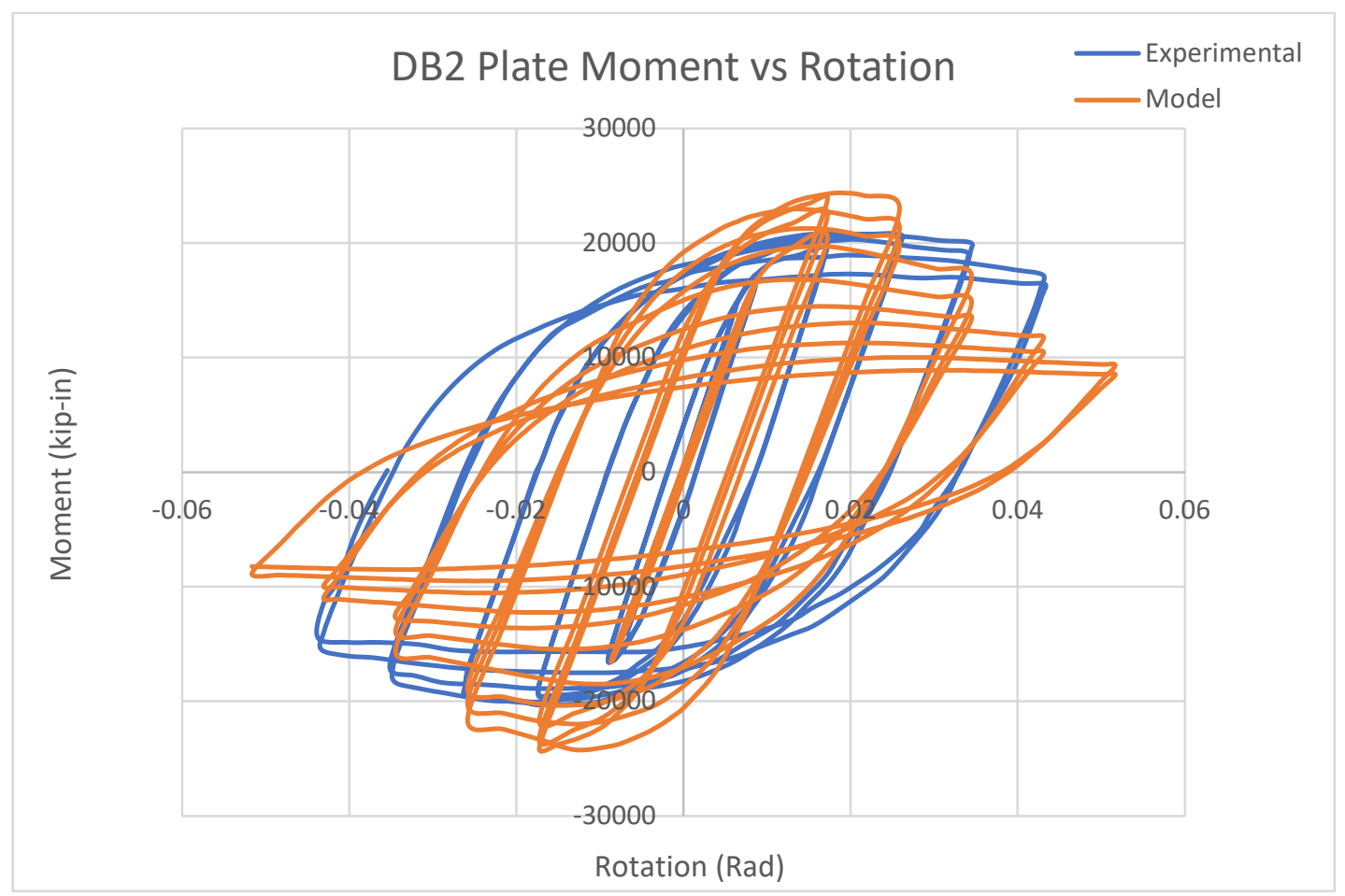

3.35 DB2 Plate Model Moment vs. Rotation Plot 


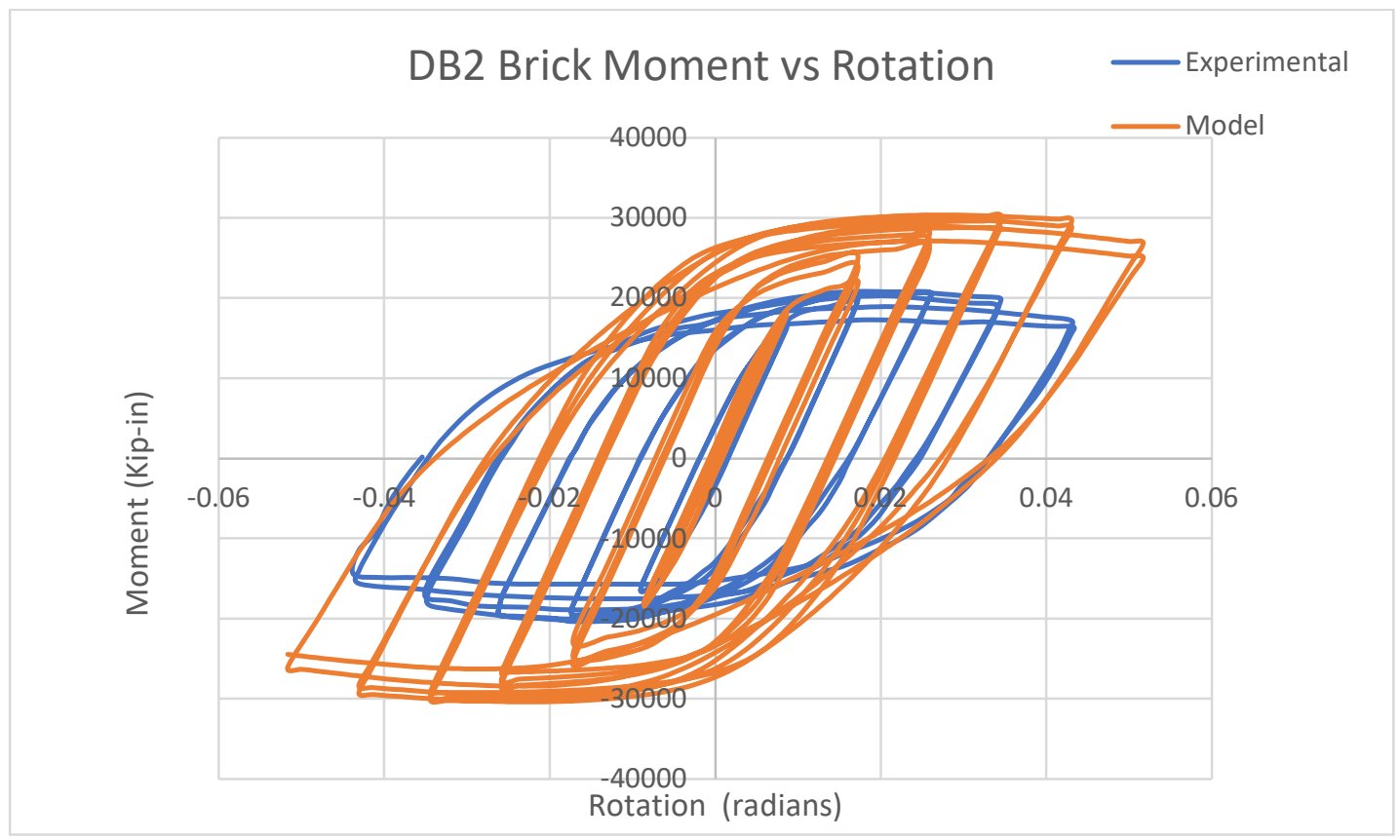

Figure 3.36 DB2 Brick Model Moment vs Rotation Plot

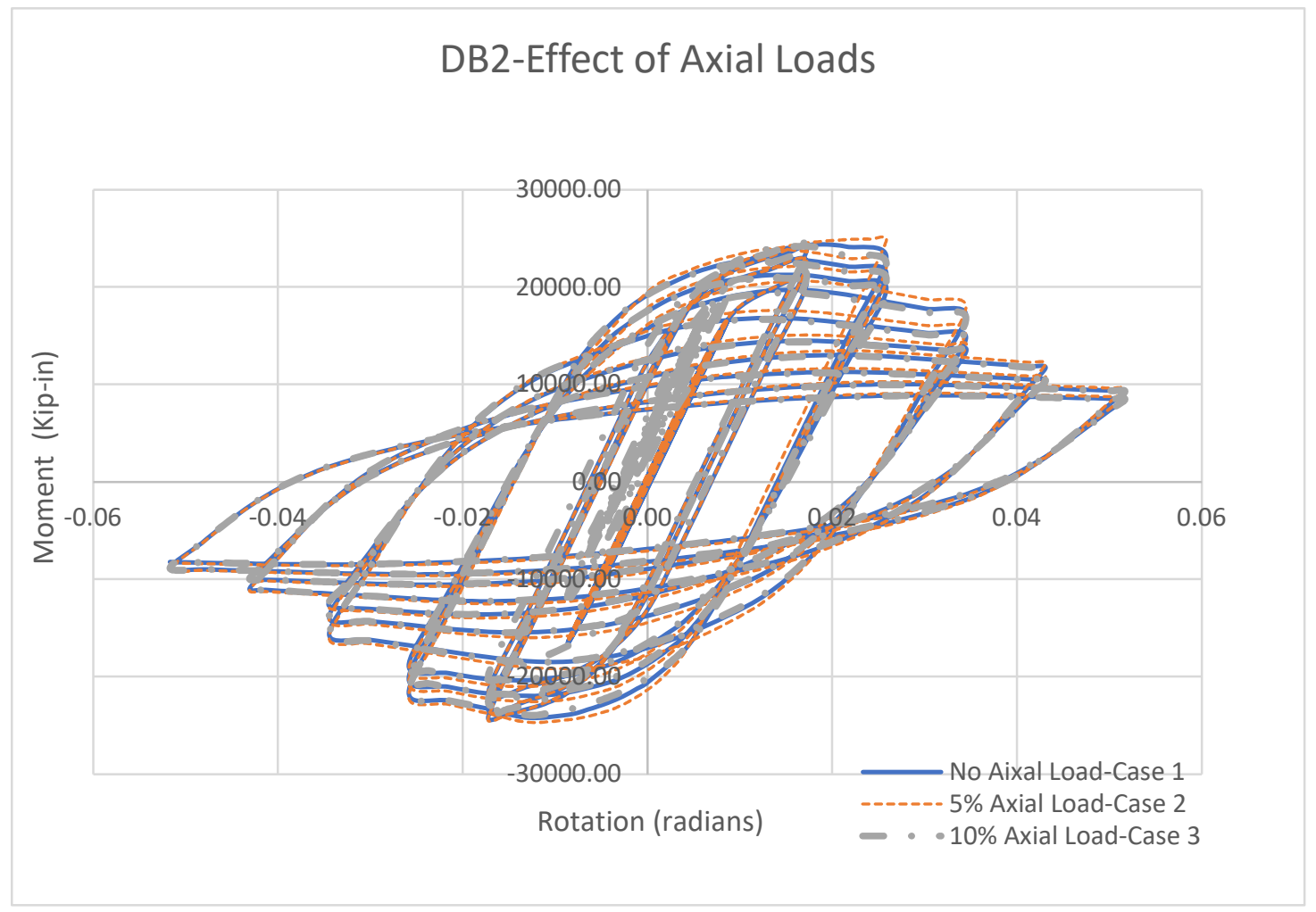


Figure 3.37 Comparison of cyclic behavior of cases with different axial load (DB2: W14x426 column, W36x150 beams)

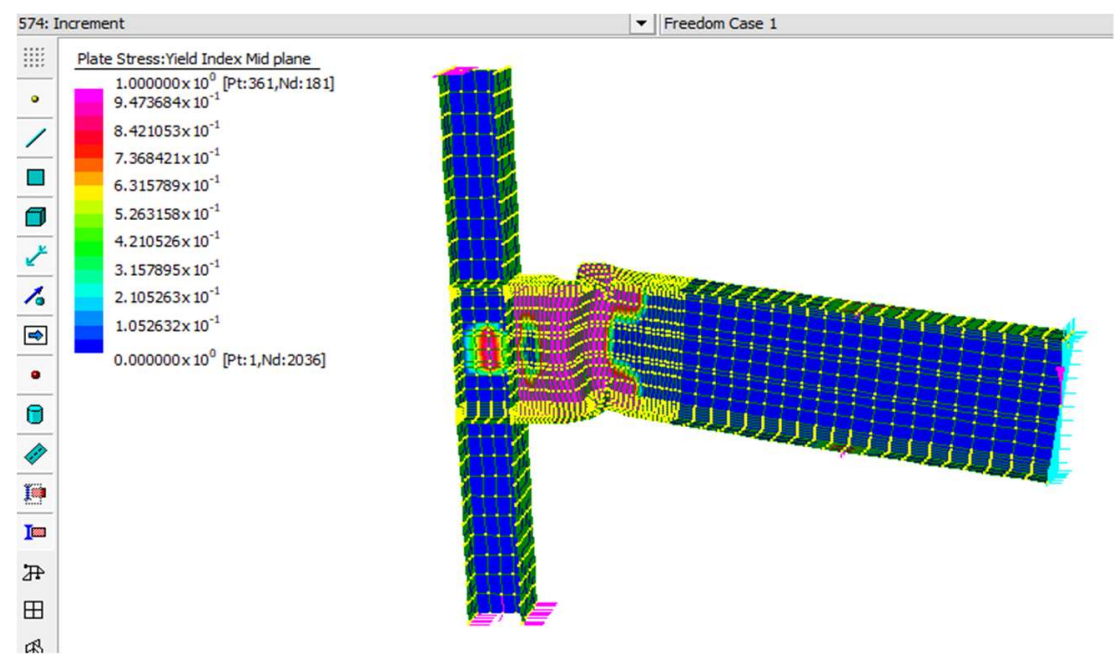

(1)

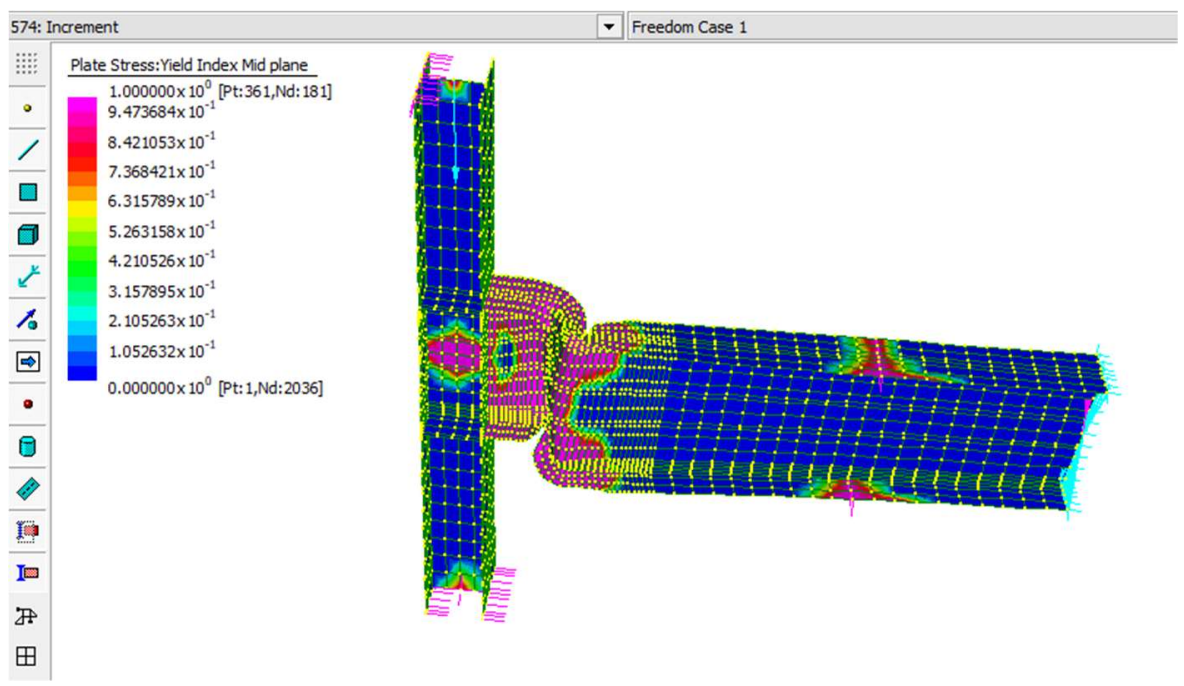

(2) 


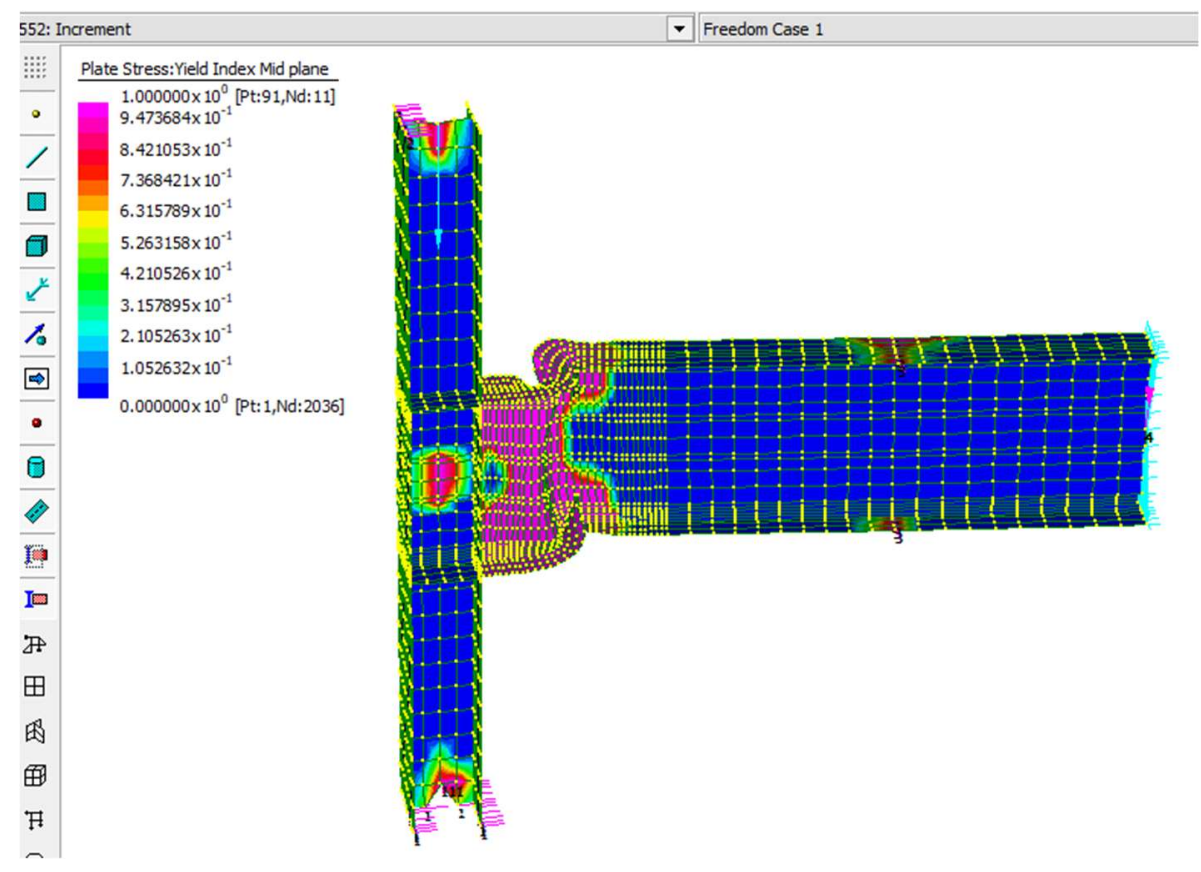

(3)

Figure 3.38 Yield Index Contour for DB2 Models with various axial load effects:

(1) 0\%Axial Load (2) 5\% Axial Load (3) 10\% Axial Load

\section{Chapter 4}

FEA of Steel Moment Resisting Frame with RBS connection

\subsection{General FEA model description}

A steel moment resisting frame (SMRF) with RBS connection was modelling in Stand7 with the plate/shell element. The global model is shown in Figure4.1. On the 
other hand, a SMRF with the same member sizes was created to compare the result of finite element analysis to show the seismic performance of this Prequalified Connection base on the design requirement of AISC 341 (2017) seismic previsions. The base model named FrameBase was created without RBS and it is a one bay one story frame with columns bottom fixed boundary condition. The boundary condition followed the ASCE 7-16, chapter 12. seismic requirement for nonlinear time history analysis for steel moment resisting frame. Lateral bracings were applied to the beam span away from the RBS (protected zone) to prevent out of plane movement which can cause excessive lateral torsional buckling. Another two models named FrameDB2 were moment frame with constant cut RBS and radius cut RBS, respectively.

\subsection{Translational mass determination}

The translational mass which was applied to the structure was determined by the following equations

$$
\begin{aligned}
& w=\sqrt{\frac{k e}{m}} \\
& w=(2 * \pi) / T \\
& m=\frac{k e}{\left(2 * \frac{\pi}{T}\right)^{\wedge} 2}
\end{aligned}
$$

where

$$
w=\text { angular frequency }
$$


$m=$ translational mass

$k e=$ effective lateral stiffness of the system

$T=$ natural period of the system

In order to obtain the effective stiffness of the structure, it is idealized as two lumped masses supported by the two columns with stiffness $\mathrm{k}$ in the lateral direction. Therefore, the lateral stiffness of the system is equal to the sum of the stiffness of individual columns, according to Researcher Chopra (2014).

And the stiffness of the frame with a beam with realistic stiffness as we assumed, was calculated by the following equation:

$$
k=\frac{24 E_{c}}{h^{3}} \frac{12 \rho+1}{12 \rho+4}
$$

where $\rho=\left(\mathrm{EI}_{\mathrm{b}} / \mathrm{L}\right) /\left(2 \mathrm{EI}_{\mathrm{c}} / \mathrm{h}\right)$, and it is the beam to column stiffness ratio ;

$k=$ lateral stiffness of the structure;

$E=$ Modulus of Elasticity in ksi;

$I_{c}=$ Moment of inertia of the column;

$L=$ length of beam;

$h=$ column height;

And For the column sections corresponding to the model DB2, E=29000ksi, $\mathrm{I}_{\mathrm{c}}=6600$ in^4, $\mathrm{h}=136$ in. As a result, the lateral stiffness $k$ was determined to be $1010285 \mathrm{kip} / \mathrm{in}$ for the Frame with RBS, 1139874 kip/in for the Frame with full beam section. The translational mass is 2779050.3 LB for frame with RBS, while 3135516.4 LB for frame with 
full beam section. Details on the procedure of node mass calculations with can found in Appendix Table A.1.

Translational nodal masses were applied to the column tops representing the total story weight. The natural period, nevertheless, depends on the seismic mass that participates during time history analysis, which can be obtained by the bill of materials with average component from strand7. The seismic mass that participated in the response of model FrameDB2 is shown in Figure 4.2. The expected natural frequency of the structure determined by using the Natural Frequency Solver in strand7 which will be discussed below.

\subsection{Natural Frequency of Frame DB2}

The natural frequency of the frame model was attained by conducting the linear Natural Frequency Analysis in Strand with the natural frequency solver. Table 4.1 show the results of the Natural Frequency attained from the solver for both moment frame models. By comparing the results for both models, it clearly showed that the natural frequency for the RBS frame is about $0.89 \mathrm{~Hz}$ and it is bigger compared to the frame with full beam section which is close to $1.2 \mathrm{~Hz}$. This is expected due to the lighter mass of the RBS moment frame. Additionally, 4 modes shapes from the eigenvalue analysis from the solver were presented in Figure 4.2. These modes were used as references in the time history analysis (THA) to study the failure modes of the frame. Furthermore, the natural frequency will be investigated in the nonlinear FEA time history analysis in the following session. 


\subsection{Nonlinear Time history Analysis of SMRF with Strand7 Transient Dynamic Slover}

Nonlinear time history analysis for the moment frame models was conducted by using Strand7's nonlinear transient dynamic solver. The selected ground motion with 19.95s duration was selected to use in the analysis by considering the model mesh size and relatively shorter duration time in order to ensure the running time for the analysis suitable with the allowable computer capacity. Figure 4.2 shows the ground motion used in the FEA solver with acceleration versus time steps plot. Note that the acceleration is scaled by multiplying 386 to convert the unit to be in $/ \mathrm{s}^{\wedge} 2$. Geometric and Material Nonlinearity are both considered in the analysis and 5\% added damping was used. Moreover, total of 1995 steps with 0.01 second time step were set up in the solver according to the ground motion time steps which is mentioned previously. Note that automatic sub-increment with time step reduction is used to assist model convergency as well. Only Y-direction translational mass' component was included and rotational mass was excluded in the analysis to ensure that only the translational mass' degree of freedoms were assembled. Furthermore, the Newmark Method is used for integration, which assumes a linear variation of acceleration from time $t$ to time $t+\Delta t$, according to Strand7 Reference Manual (2020).

First, the analysis is conducted by using the Linear Transient Dynamic solver to predict the general response of the moment frame subjected to the selected ground motion simulation. The displacement measured from the node which was located on top of the column representing the roof displacement from the moment frame is plotted 
versus the time steps showing in Figure 4.5. It shows the response of the moment frame oscillates about the zero displacement along in the time history since the frame remain ductile and elastic in linear analysis. It anticipates that the response of the material doesn't reach the nonlinear part of stress vs strain curve. Nevertheless, the general response of the frame is perceived, for example, the natural frequency of the frame with RBS can be predicted by measure the natural period of the response, which is about $0.8 \mathrm{~s}$. Therefore, the natural period is determined to be $1.25 \mathrm{~Hz}$, which is about $6 \%$ difference from the natural period that is predicted from the natural frequency solver. Yet the response and will be used to compared with the response from nonlinear analysis to investigate the geometric and material nonlinearity effect of the beam column connection for the RBS frame model and the full beam section frame model.

The graphs are shown in Figure 4.4 and Figure 4.5 accordingly and the difference for the two frames can be detected to show the effect of the RBS to the frame's seismic nonlinearity. The responses from both graphs show significant degree of nonlinearity of the frame by presenting the roof displacement doesn't oscillate about the zero displacement anymore along the time history proceeds. The frame changes ductility and exhibits plastic deformation due to the material and geometric nonlinearity were considered in the analysis.

\subsection{Lateral Bracing Force Estimation}

Lateral Bracing was applied to the beam flanges in the moment frame models to prevent excess out of plane movement due to the local and torsional lateral buckling of the beam flanges. As result, the displacements of X-direction were restraint to be zero for 
the nodes at the locations of the beam top and bottom flange of the beam as shown in Figure 4.7. For the moment frame with RBS, the required lateral restraint force was 26.1 kips, which was predicted by Strand7 in nonlinear dynamic analysis, and that was corresponding to the maximum roof displacement of 4.44 inches which was determined in the previous session. In addition, the required lateral restraint force was determined to be 59.8 kips in the nonlinear static pushover analysis with 6 inches displacement cyclic load magnitude. These maximum lateral forces for two types of analysis are shown in Figure 4.8. The nonlinear static analysis will be covered more in depth in the following session. Note that these required lateral forces were based on infinite stiffness presented for the beam flanges. Though in fact infinite stiffness is not likely to achieved in steel moment frame design, the stiffness of the beam flanges can be adjusted to be certain value in order to have better estimations of the required lateral forces.

Four cases were established to investigate the how much lateral forces are required corresponding to the applied stiffness of the beam flanges. Case 1 and Case 2 are with $52.2 \mathrm{k} /$ in spring stiffness applied to the beam flange for time history and pushover analysis in order to allow the beam to have 0.5 inches of lateral displacement. While Case 3 and Case 4 are with $26.1 \mathrm{k} /$ in spring stiffness applied to the beam flanges to allow the beam to have 1 inch of lateral displacement for both time history and pushover analysis. The results of maximum lateral forces were tabulated in Table 4.3 and compared to the result with the infinite stiffness that described previously. By inspecting the result, it reveals that the maximum lateral forces of beam flanges change after the spring stiffness was applied to the models both in nonlinear time history analysis and nonlinear pushover analysis. The tabulated maximum lateral forces are the sum of the peak lateral forces of 
total of four lateral braced nodes on the beam top and bottom flanges. While in case1with the applied stiffness to reach allowable 0.5 in stiffness of the beam flange, the required lateral force is $2.71 \mathrm{kip}$ from nonlinear time history analysis and it is about $10.4 \%$ of the maximum lateral force with infinite stiffness. Furthermore, in case 3 the required lateral force is 36.6kip from nonlinear static pushover analysis and it is about $61.2 \%$ of the maximum lateral force with infinite stiffness. For the case 2 and case 4 , the calculated required maximum lateral forces are also showing big percentage of reduction compared to the maximum lateral forces corresponding to the beam flanges with infinite stiffness. The force histories of both analysis cases are presented in Figure 4. Since the design of lateral bracing according to the design code AISC 358 doesn't specified the lateral stiffness of the beam flanges, the designed lateral bracing force can be overestimated. As a result, by changing the stiffness of the beam flanges accordingly, the required lateral force can be estimate from Strand7 and it can be an effective tool to evaluate the required lateral forces complies with the steel moment resisting frame design codes.

\subsection{Nonlinear Static Analysis (NSA) of RBS Moment Frame Model}

To investigate the failure mechanism and frame instability. Nonlinear static pushover analysis was conducted by using the nonlinear static solver with geometric nonlinearity and material nonlinearity were considered. Cyclic load following the ATC24 loading protocol as described previously is applied to the roof level with displacement control. Other solver parameters are set to be the same as the pushover analysis conducted for RBS connections as mentioned in the previous chapter. The analysis 
stopped at the 6 in displacement load which was believed to be adequate to inspect the failure mode of the moment frame and due to the limitation of computer CUP capacity. The failure mode at the end of the pushover analysis is shown in Figure 4.7. Flange local buckling and lateral torsional buckling can be detected and twisting of columns are also presented. Base shear versus displacement plot is generated and the maximum magnitude of base shear can be determined to be 487 kips when the roof displacement of the structure is around $4 \mathrm{in}$. Occurrence of instability of the structure is anticipated if the magnitude to the cyclic loading increases.

Base shear from nonlinear transient dynamic analysis for moment frame with RBS and with full beam section are plot versus displacement for comparison in Figure 4.10 and Figure 4.11. Bigger magnitude of maximum base shear was shown in the plot for the moment frame with RBS, which is 546.9 kip when displacement reaches $4.41 \mathrm{in}$, while the maximum base shear is 506 kip with roof displacement of 5.04 in for the full beam section frame. As a result, the RBS play a role at changing the nonlinear base shear response of the moment frames. In other words, it reduced the roof displacement when the frame is subjected to the same selected ground motion, though the base shear increases by about $8 \%$. On the other hand, lower maximum base shear was generated in pushover analysis compared to the one extrapolated from dynamic time history analysis because the load is gradually applied to the structure in a predictable manner by following the loading protocol, therefore, sudden rupture of plate elements won't occur. Furthermore, nonlinear dynamic time history analysis generated bigger roof displacement exceed 6 in, hence, bigger base shear can be presumed. Note that the difference of maximum base shear between these two types of analysis is about $10 \%$. 


\subsection{Incremental Dynamic Analysis}

In the interest of inspecting the possible limit states of the moment frame with RBS, incremental dynamic analysis was performed with 10 ground motions with increase scales of the selected ground motion for nonlinear dynamic time history analysis previously. Analysis stopped in three and a half $(3.5 \mathrm{x})$ times the selected ground motion due to the divergency of the solution with the deformation exceed the allowable state which set up in the solver. Peak Acceleration with unit of acceleration of gravity (g) is plotted versus maximum roof displacement in Figure 4.12 for each nonlinear dynamic analysis with the different scaled ground motion. From the result, it unfolds that the roof displacement magnifies as the ground motion becomes stronger with greater peak ground acceleration. Moreover, ultimate limit state of the structure can be presumed based on the response which shown in Figure 4.12. It shows that possible collapse with big degree of deformation when the PGA is reaching $1.45 \mathrm{~g}$.

Beam web lateral torsional buckling occurrence can be verified by looking at the plot of the out of plane lateral displacement of the beam web time history. At the beginning of the simulation, the RBS beam section didn't exhibit buckling with no sign of lateral out of plane displacement, as shown in Figure 4.13 and 4.14 for cases with scales of 2.1x and 2.2x PGA of selected ground motion respectively. As the simulation proceeds, displacement of the beam web rises when the time step reaches 4 seconds for both cases. Therefore, incremental dynamic analysis conducted by using nonlinear 
transient dynamic solver can adequately predict the buckling state and as well as the severity of the buckling for each ground motion shaking simulation.

Table 4.1 FEA Natural Frequency Analysis Results for Frame with Full Beam Section

\begin{tabular}{|l|l|l|l|}
\hline $\begin{array}{l}\text { FINAL FREQUENCY } \\
\text { RESULTS }\end{array}$ & & & \\
\hline Mode & Eigenvalue & Frequency (rad/s) & Frequency (Hz) \\
\hline 1 & $3.11133486 \mathrm{E}+01$ & $5.57793408 \mathrm{E}+0$ & $8.87755781 \mathrm{E}-01$ \\
\hline 2 & $5.67143740 \mathrm{E}+02$ & $2.38147799 \mathrm{E}+01$ & $3.79023993 \mathrm{E}+00$ \\
\hline 3 & $4.71704214 \mathrm{E}+05$ & $6.86807261 \mathrm{E}+02$ & $1.09308771 \mathrm{E}+02$ \\
\hline 4 & $5.06204656 \mathrm{E}+05$ & $7.11480608 \mathrm{E}+02$ & $1.13235656 \mathrm{E}+02$ \\
\hline
\end{tabular}

Table 4.2 FEA Natural Frequency Analysis Results for Frame with RBS

\begin{tabular}{|l|l|l|l|}
\hline $\begin{array}{l}\text { FINAL FREQUENCY } \\
\text { RESULTS }\end{array}$ & & & \\
\hline Mode & Eigenvalue & Frequency (rad/s) & Frequency (Hz) \\
\hline 1 & $5.56859772 \mathrm{E}+01$ & $7.46230375 \mathrm{E}+00$ & $1.18766253 \mathrm{E}+00$ \\
\hline 2 & $6.54150676 \mathrm{E}+02$ & $2.55763695 \mathrm{E}+01$ & $4.07060563 \mathrm{E}+00$ \\
\hline 3 & $7.65445291 \mathrm{E}+05$ & $8.74897303 \mathrm{E}+02$ & $1.39244230 \mathrm{E}+02$ \\
\hline 4 & $8.45969745 \mathrm{E}+05$ & $9.19766136 \mathrm{E}+02$ & $1.46385327 \mathrm{E}+02$ \\
\hline
\end{tabular}

Table 4.3 Lateral Forces of Beam flanges for Frame with RBS

\begin{tabular}{|c|c|c|}
\hline & Applied Stiffness & $\begin{array}{l}\text { Max. Beam Flange Lateral } \\
\text { Force }\end{array}$ \\
\hline \multicolumn{3}{|c|}{ Nonlinear Dynamic Analysis } \\
\hline & Infinite stiffness & $26.1 \mathrm{kip}$ \\
\hline Case 1 & $52.2(\mathrm{k} / \mathrm{in})$ & $2.71 \mathrm{kip}$ \\
\hline Case 2 & $26.1(\mathrm{k} / \mathrm{in})$ & $2.78 \mathrm{kip}$ \\
\hline \multicolumn{3}{|c|}{ Nonlinear Pushover Analysis } \\
\hline & Infinite stiffness & 59.8 kip \\
\hline Case 3 & $52.2(\mathrm{k} / \mathrm{in})$ & 36.6 kip \\
\hline Case 4 & $26.1(\mathrm{k} / \mathrm{in})$ & 39.1 kip \\
\hline
\end{tabular}




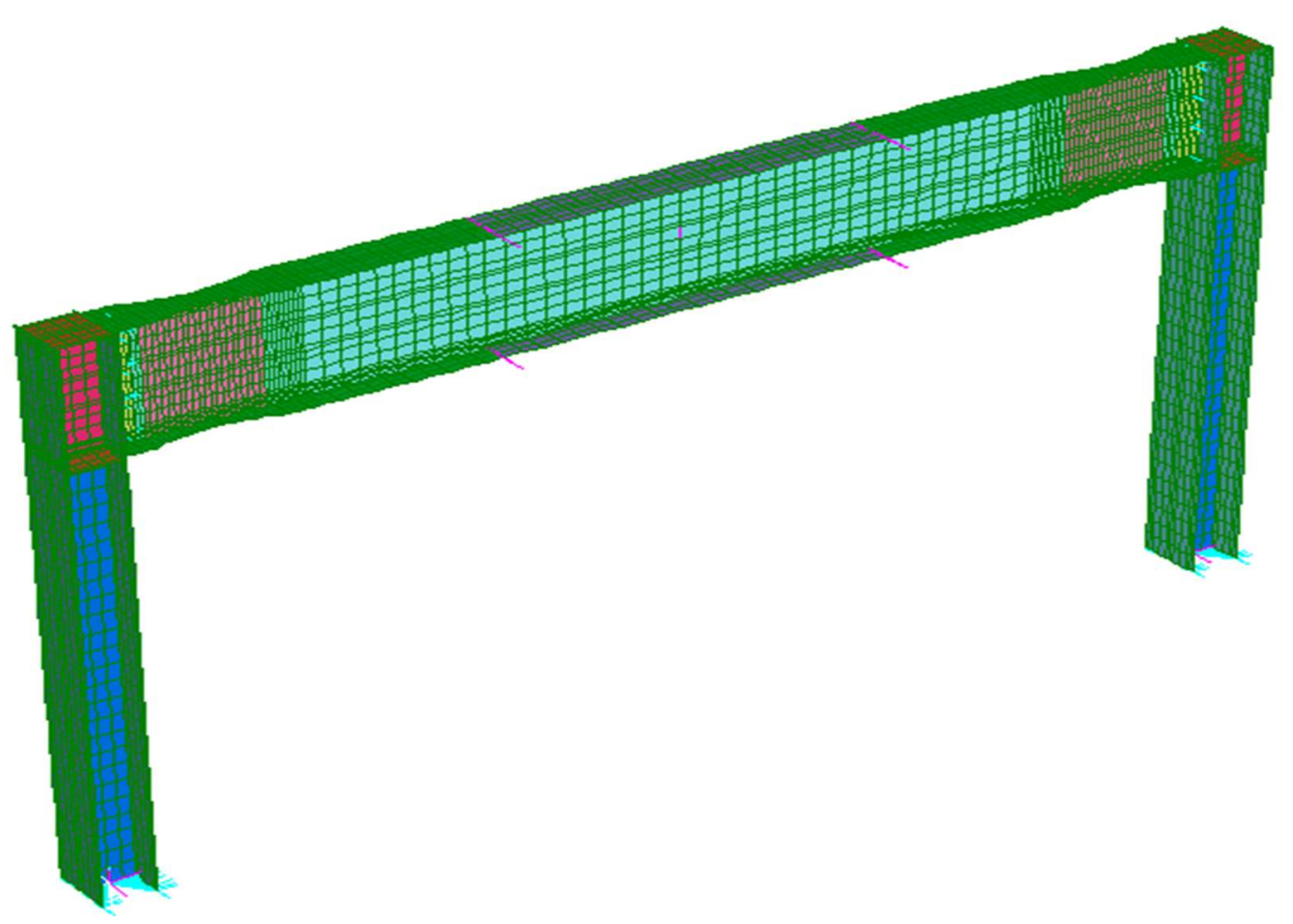

Figure 4.1 FEA Model of Steel Moment Frame with RBS

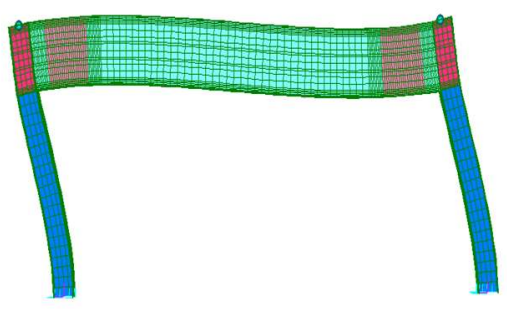

(a)

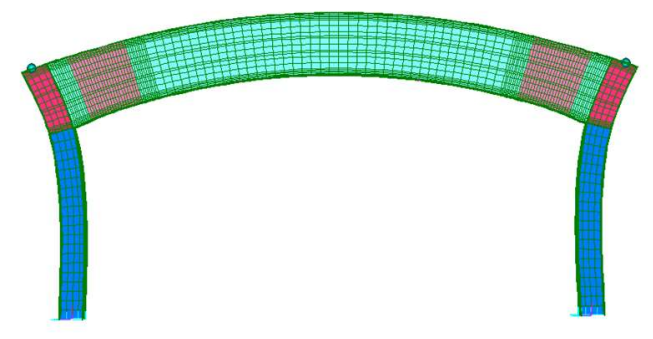

(b) 


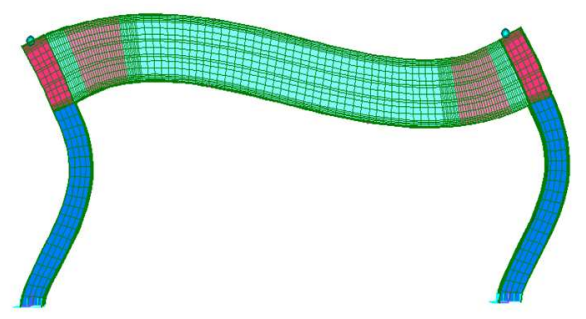

(c)

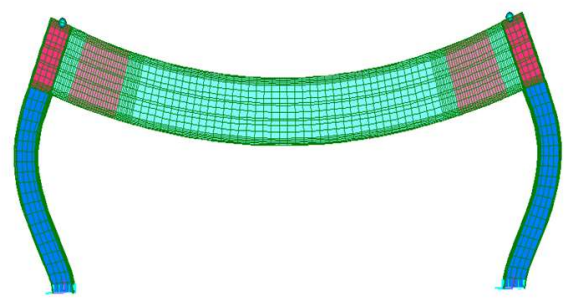

(d)

Figure 4.2 Failure modes of the FEA Frame Model

(a) First Mode (b) Second Mode (c) Third Mode (d) Fourth Mode

(Strand7 Natural Frequency Solver,2020)

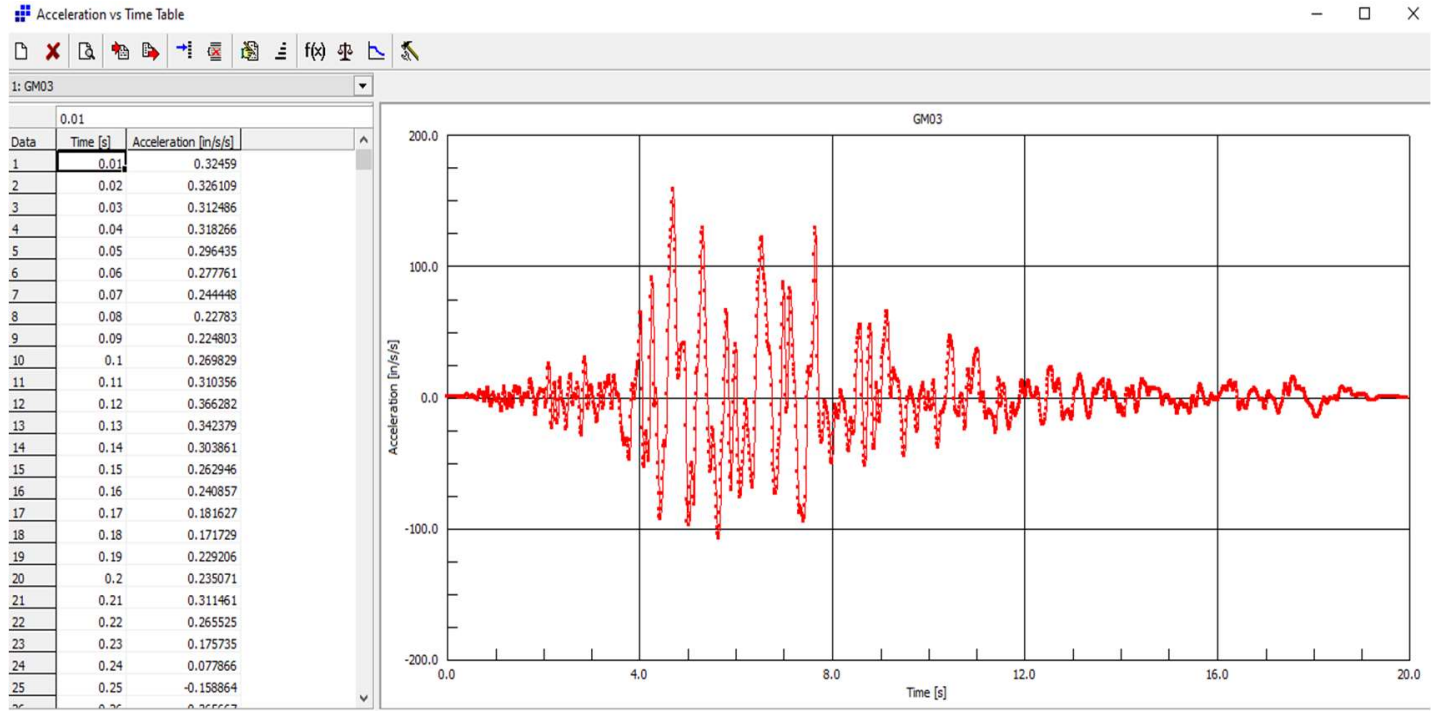

Figure 4.3 Selected Acceleration vs Time Ground Motion for Moment Frame Models 


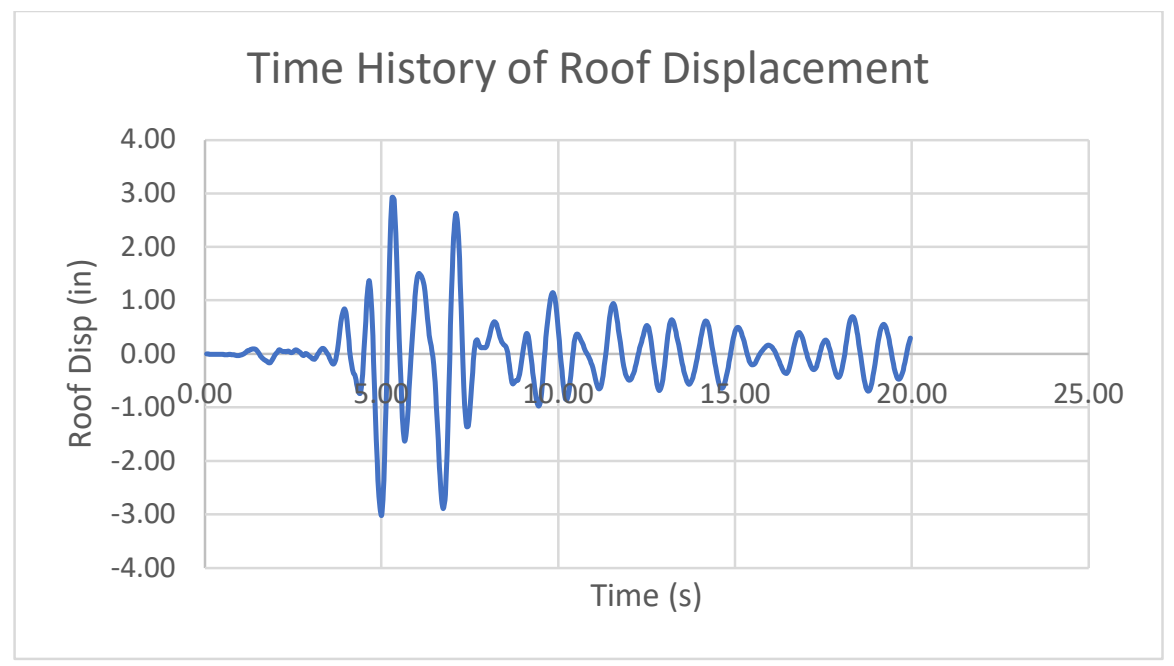

Figure 4.4 RBS Moment Frame Roof Displacement Time History

(Linear Dynamic)

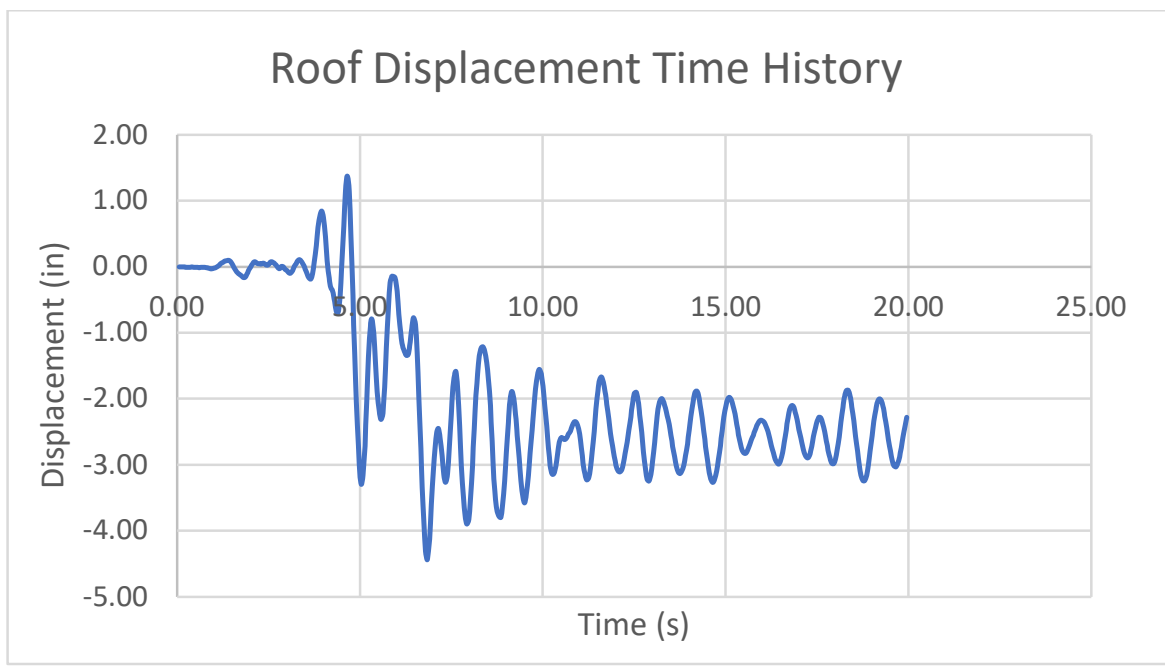

Figure 4.5 RBS Moment Frame Roof Displacement Time History

(Nonlinear Dynamic) 


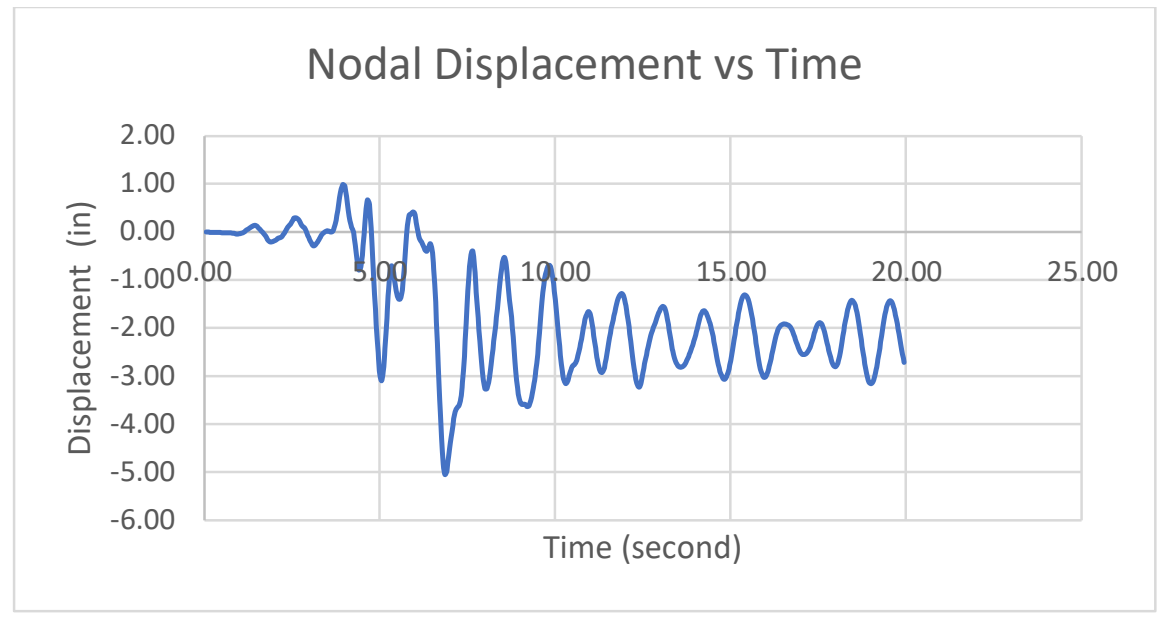

Figure 4.6 Full Beam Section Moment Frame Roof Displacement Time History

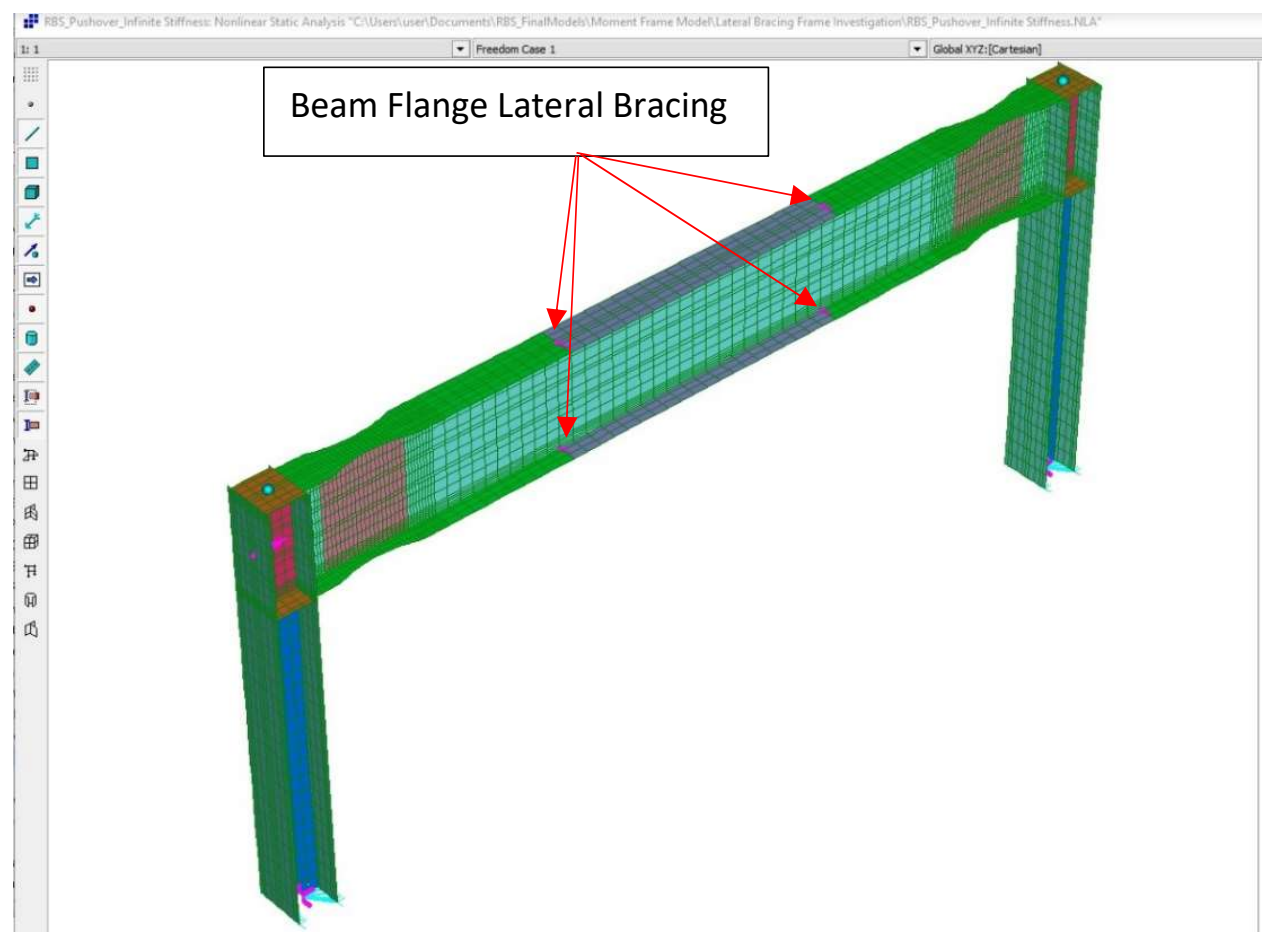

Figure 4.7 DB2 Moment Frame Beam Flange Lateral Bracing 


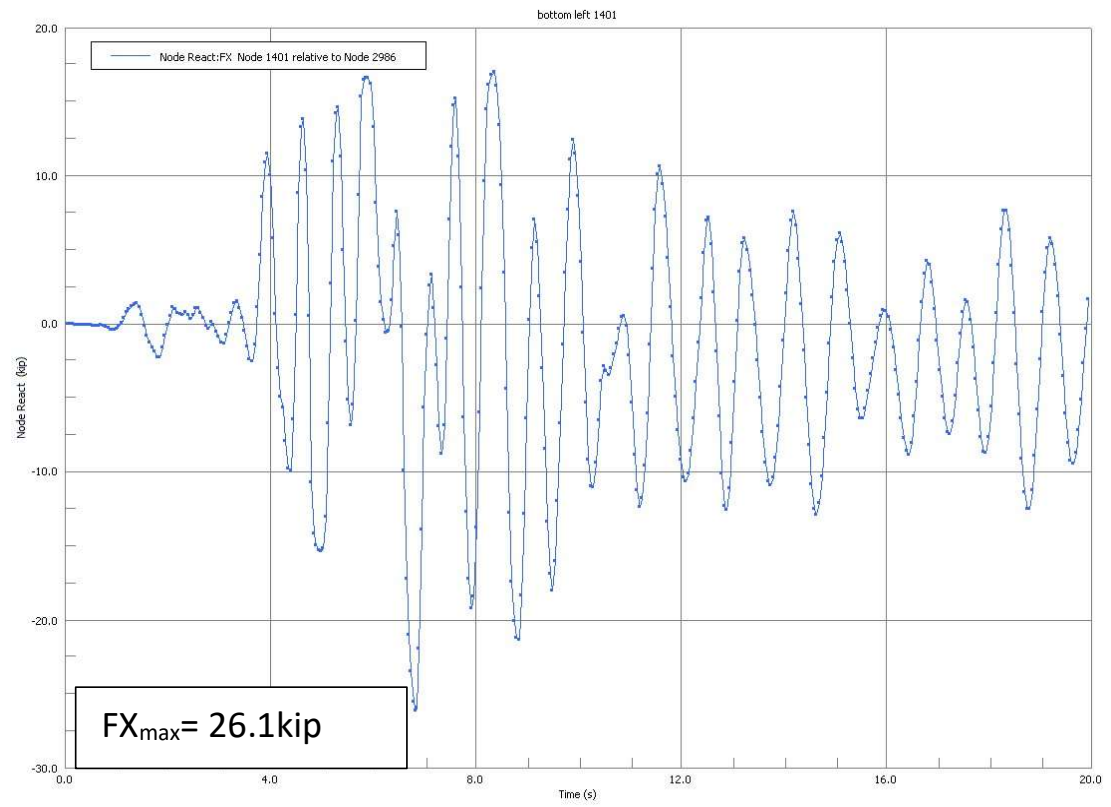

(a)

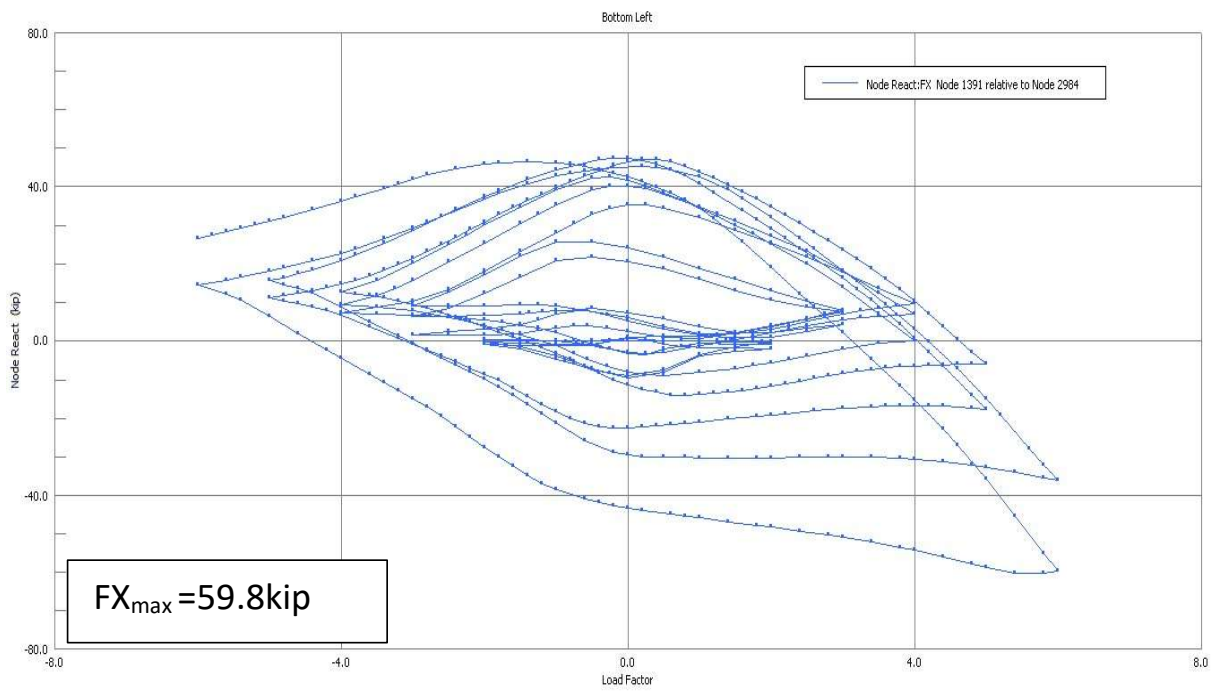

(b)

Figure 4.8 DB2 Moment Frame Beam Flange Lateral Bracing Force History:

(a)Nonlinear transient dynamic; (b) Nonlinear pushover analysis 

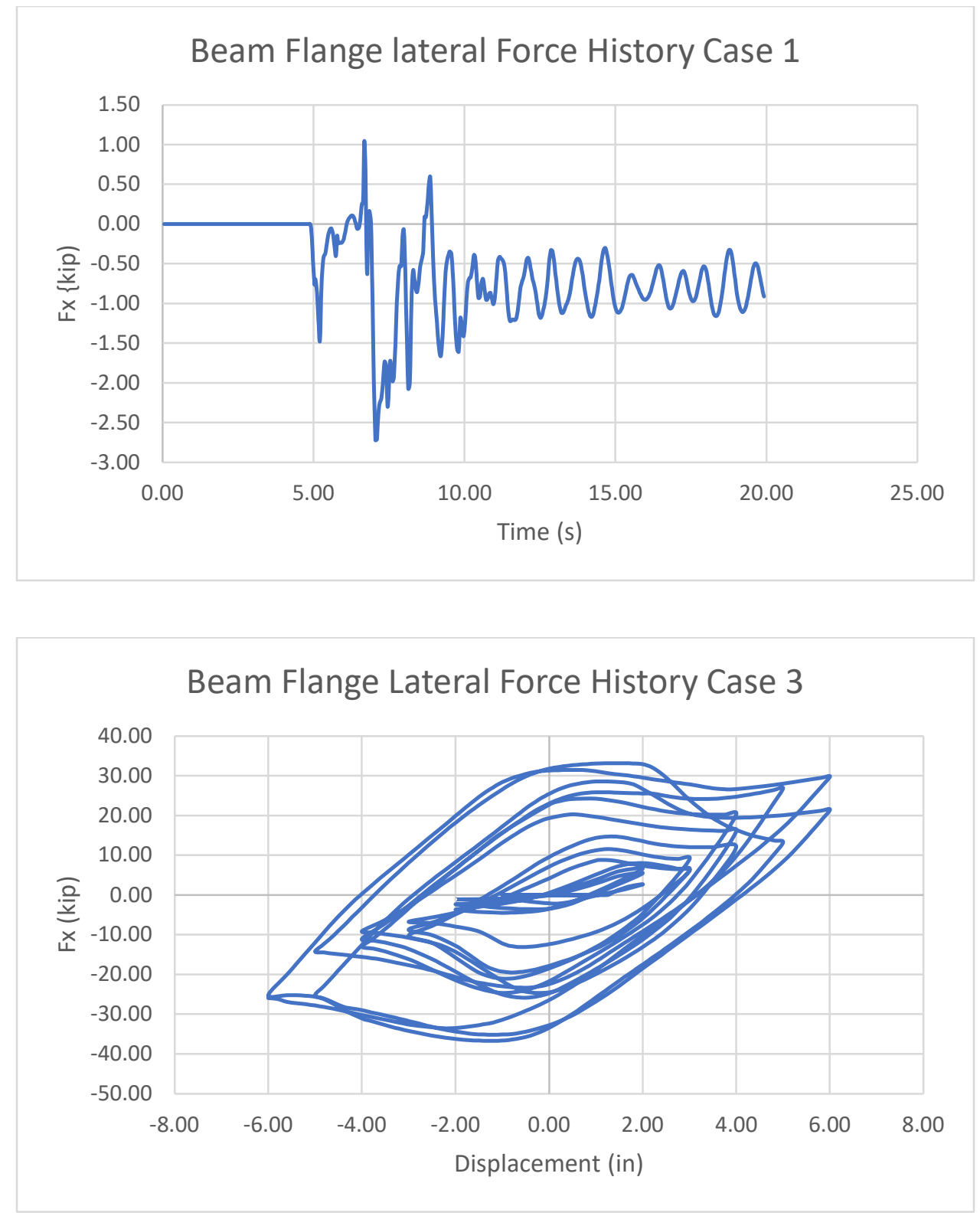

Figure 4.9 Beam flange lateral force history for case 1 (top) and case 3 (bottom) 

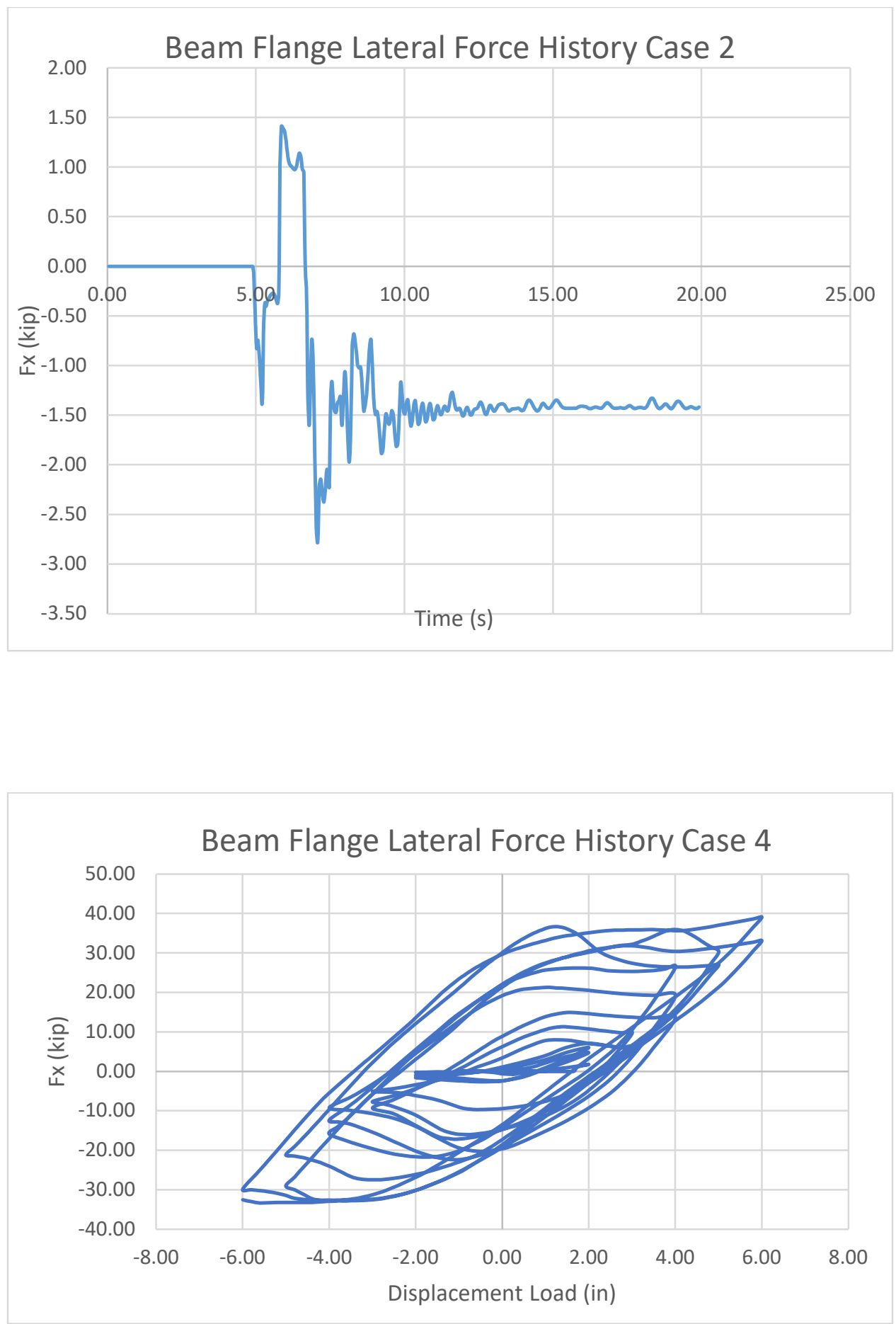

Figure 4.10 Beam flange lateral force history for case 2 (top) and case 4 (bottom) 


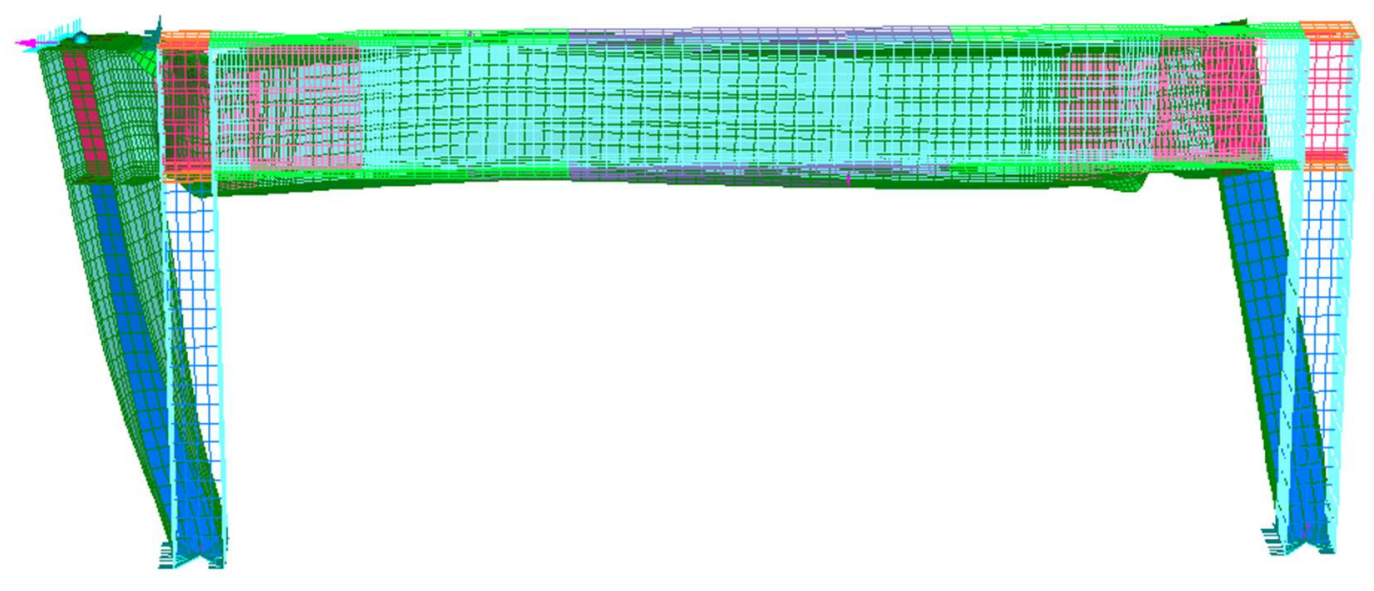

IFDUGATTONALUSE ONIYG:Chen YUAanhonol

Figure 4.11 Deformed shape of RBS Moment Frame (NSA)

(with 10\% displacement scale and undeformed shape added)

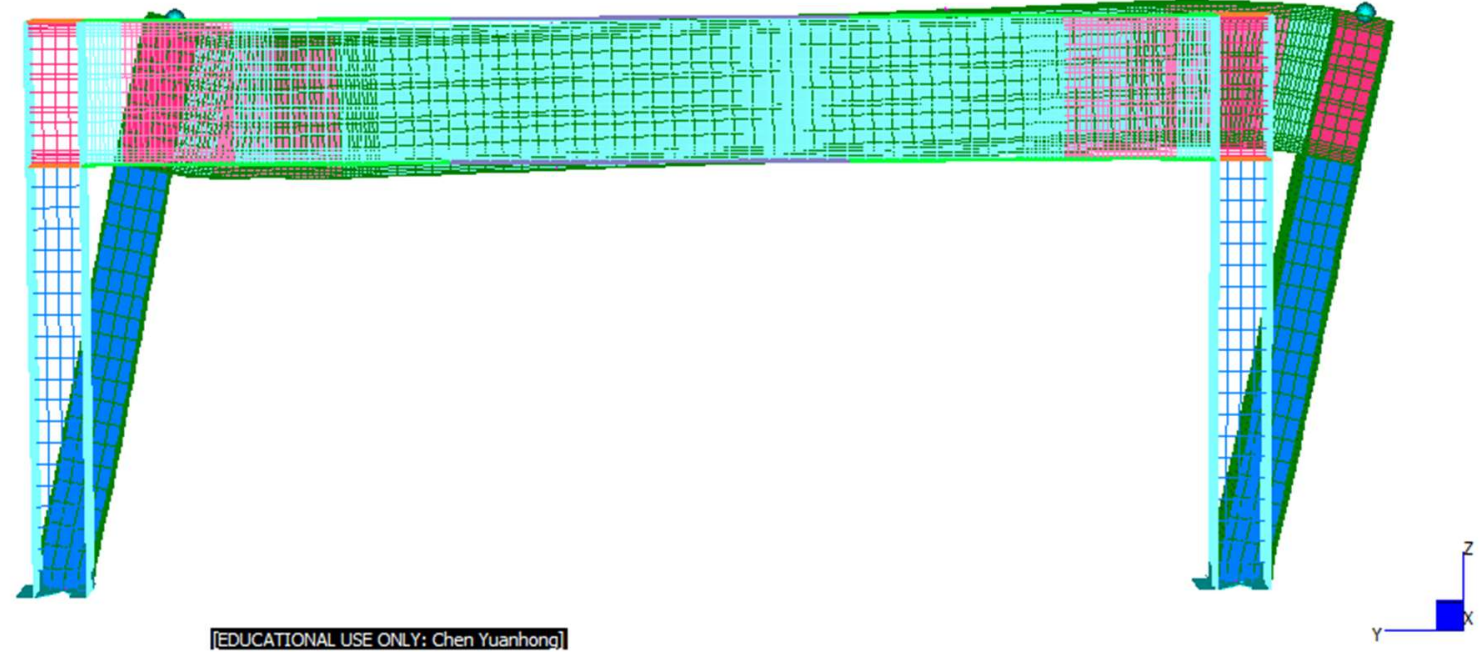

Figure 4.12 Deformed Shape of RBS Moment Frame (THA)

(with 10\% displacement scale and undeformed shape added) 


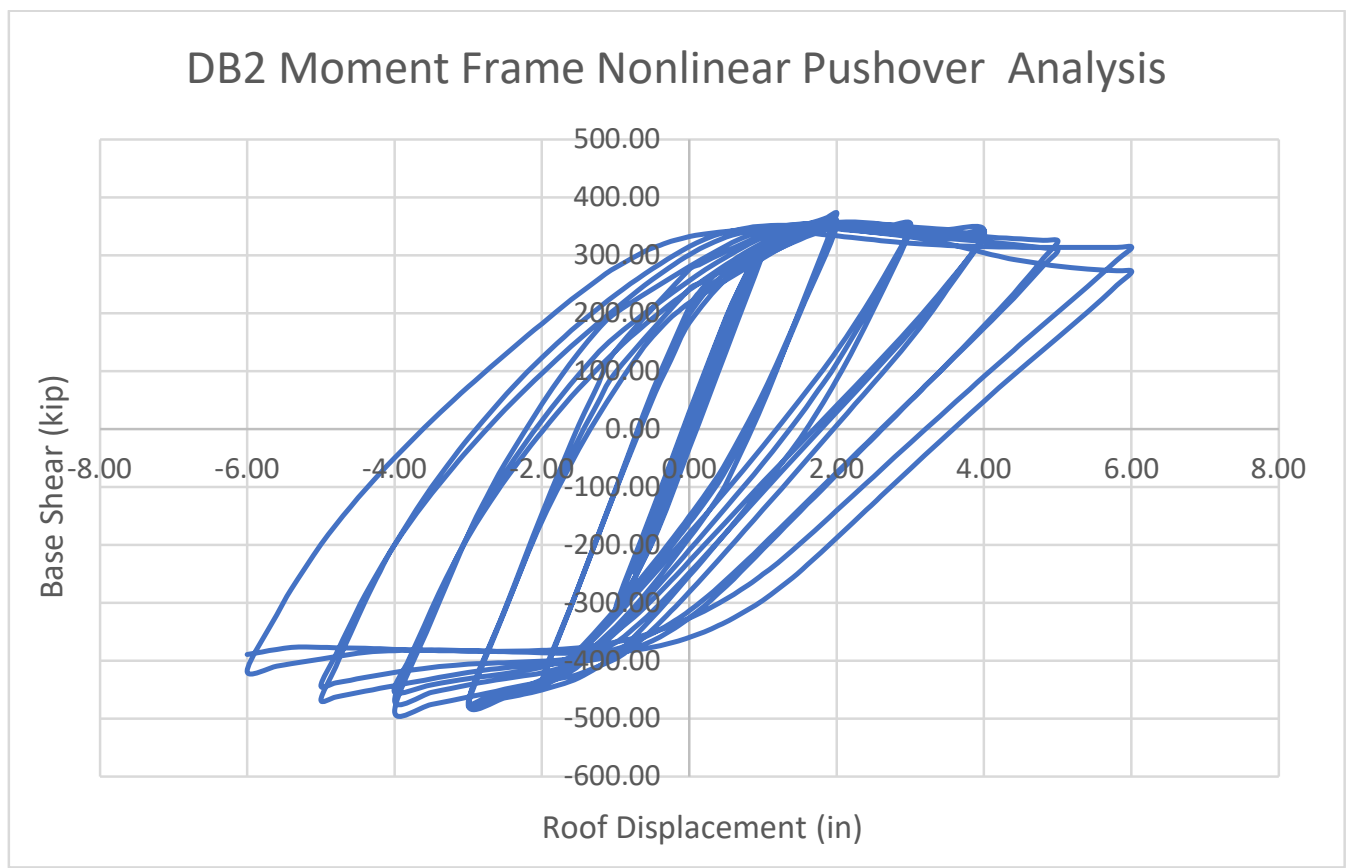

Figure 4.13 Base Shear vs Displacement (Nonlinear Pushover Analysis)

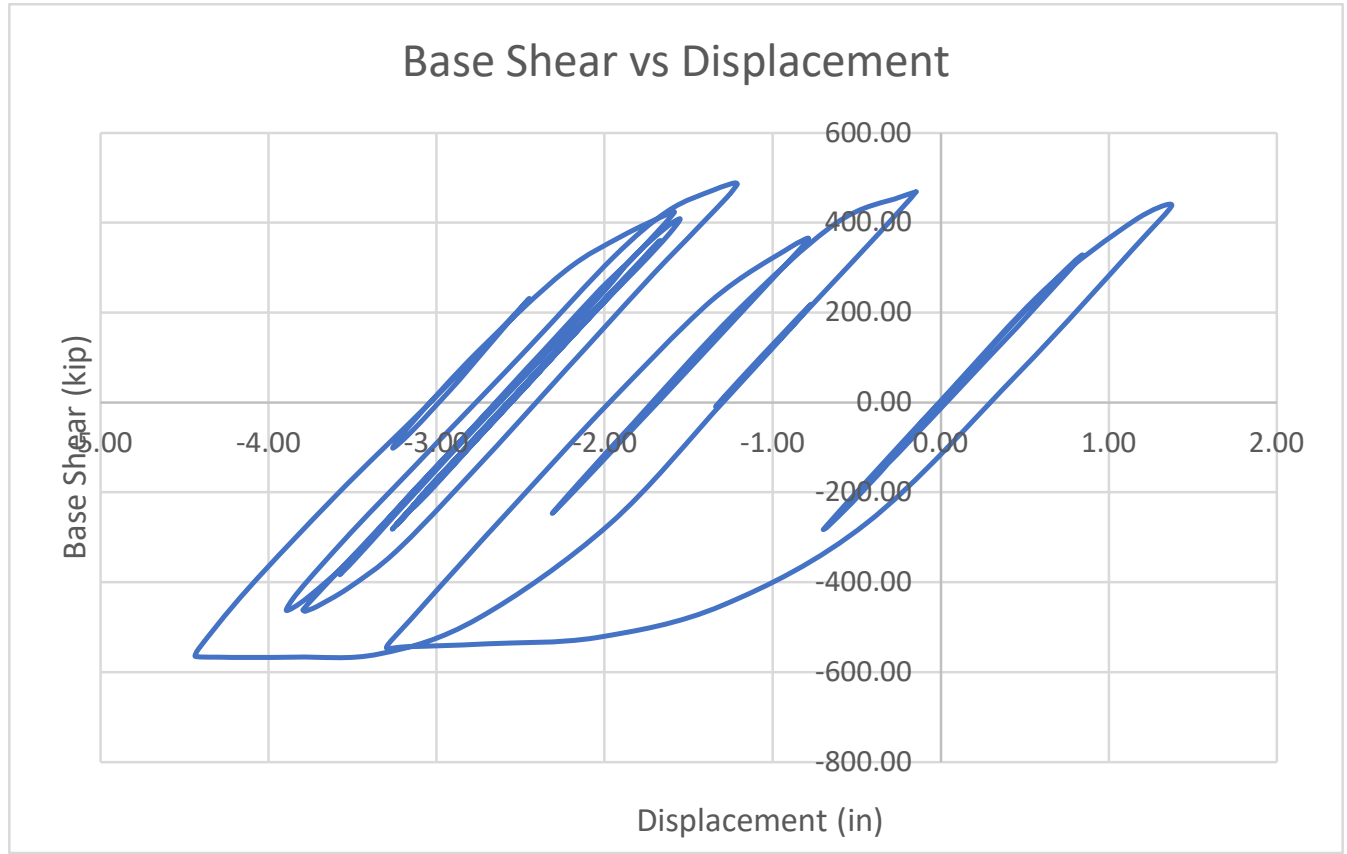

Figure 4.14 RBS Moment Frame Base Shear vs Displacement Time History 


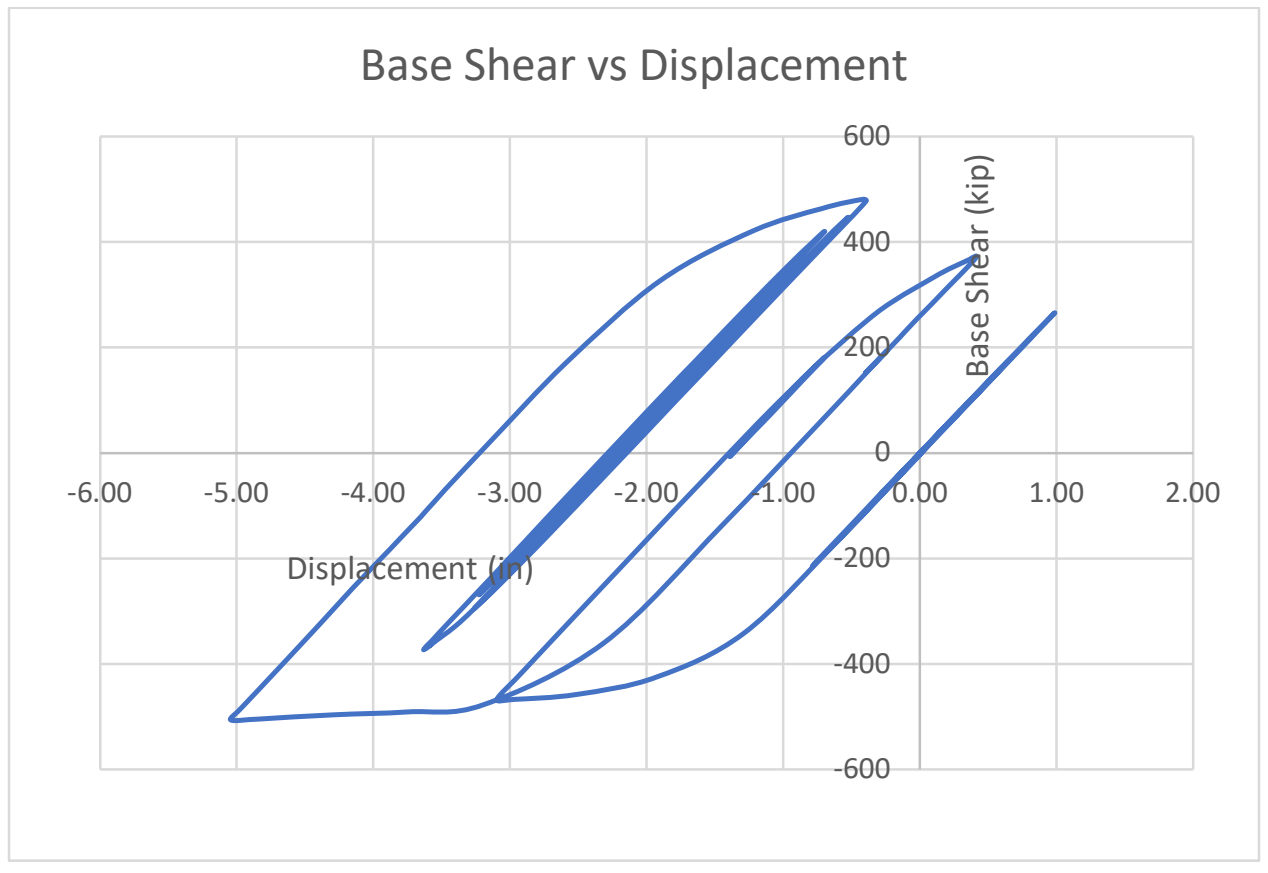

Figure 4.15 Full Beam Section Moment Frame Base Shear vs Displacement (Time History Analysis)

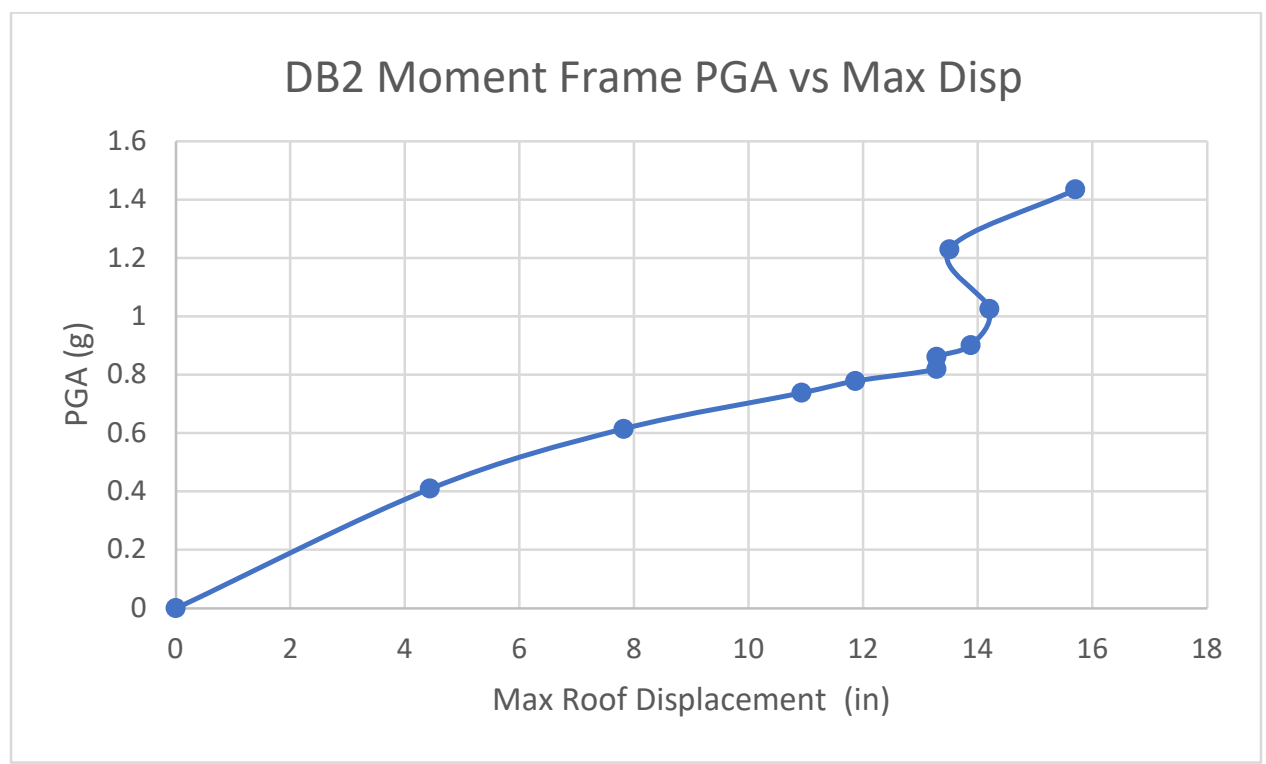

Figure 4.16 Structural Response of DB2 Moment Frame: Peak Ground 
Acceleration vs. Max. Roof Displacement

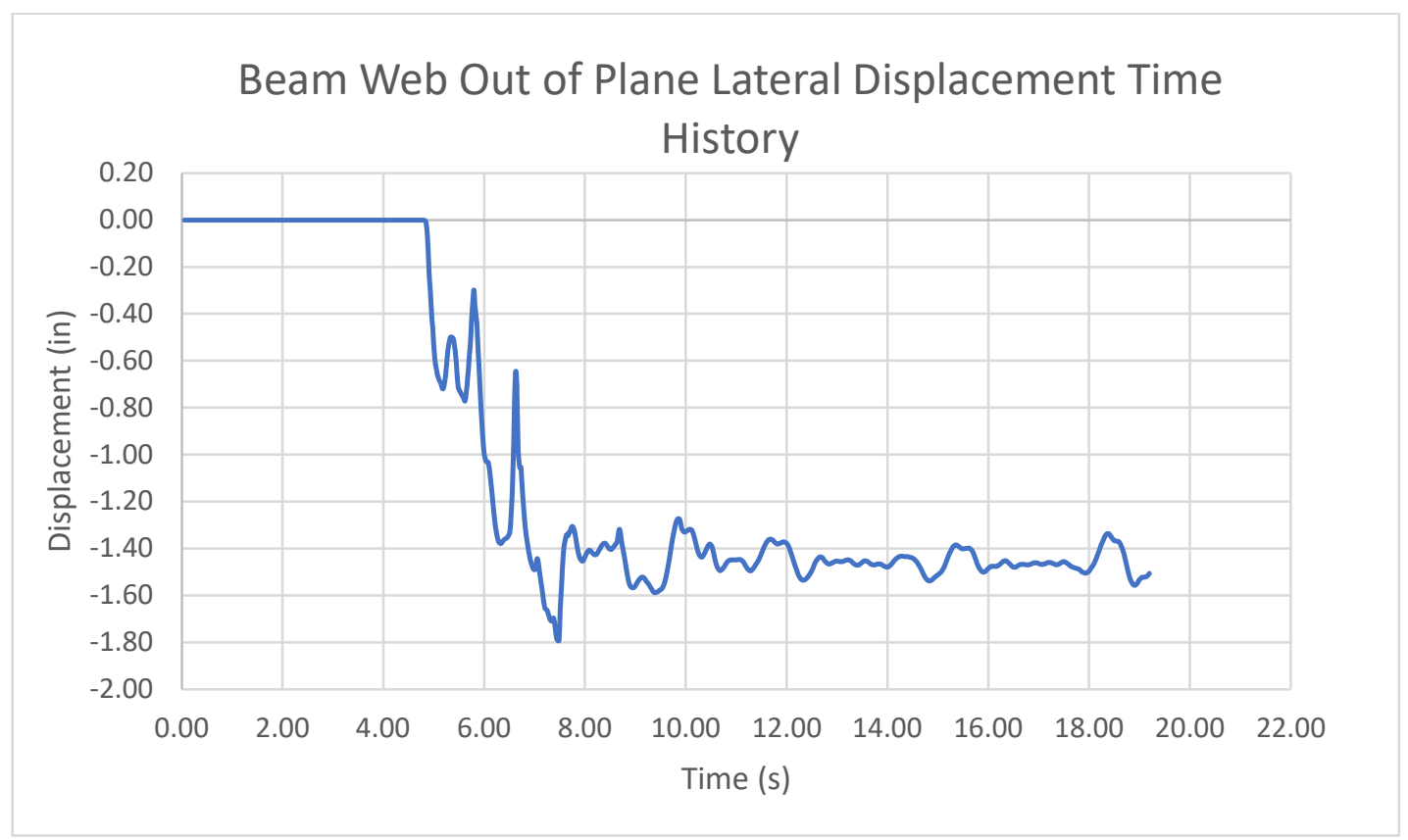

Figure 4.17 Out of Plane Lateral Displacement of Beam Web (2.1x scaled)

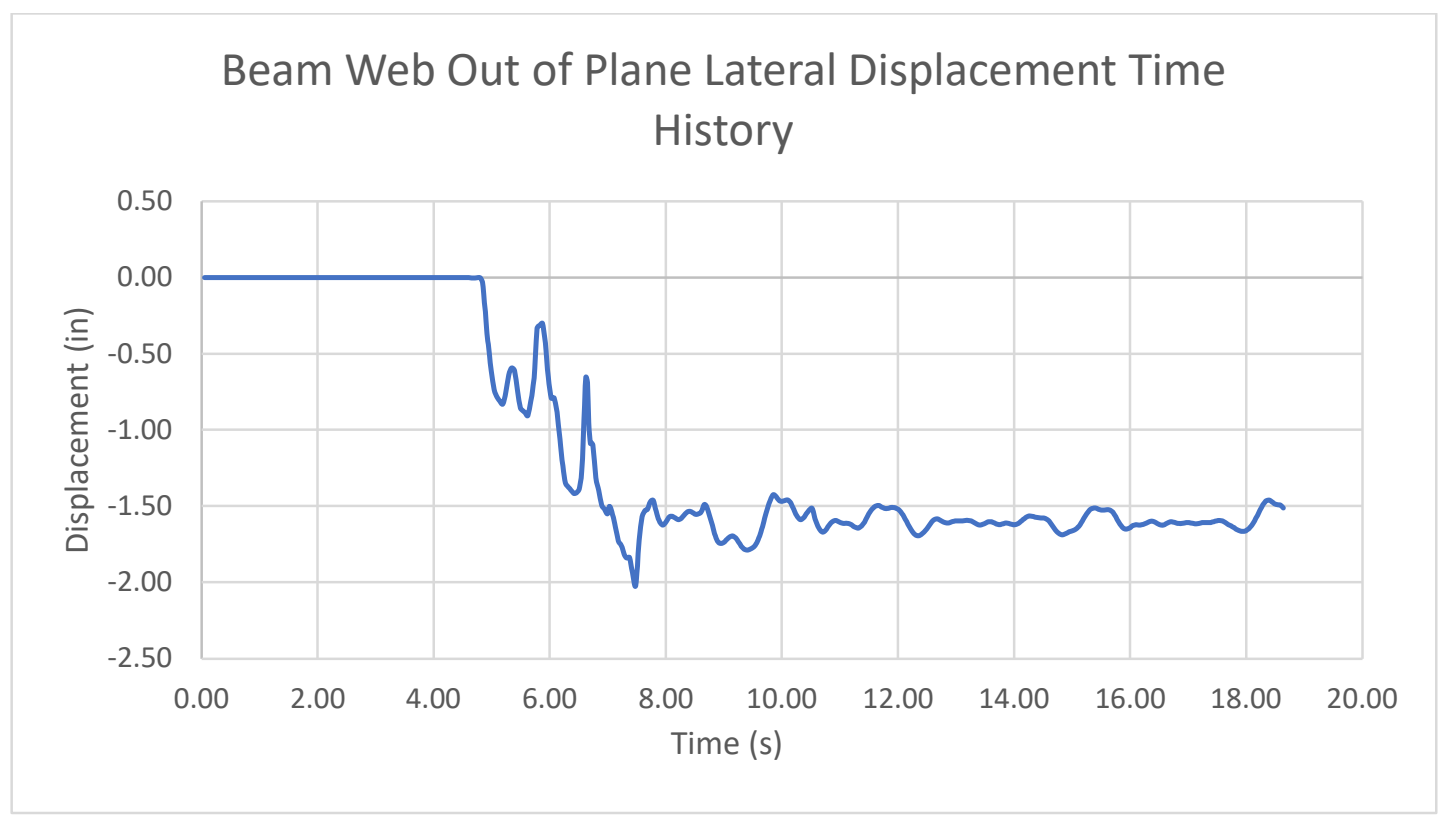

Figure 4.18 Out of Plane Lateral Displacement of Beam Web (2.2x scaled) 


\section{Chapter 5}

\section{Summary, Conclusion and Future Work}

\subsection{Summary}

Prequalified steel moment connection Reduced Beam Section (RBS) design has been tested with full scale experiments to investigate the nonlinear response and seismic performance with numerical time history analysis. Yet the cost of the full-scale experiment was beyond economical and changing of test specimen parameters is unachievable. Finite element analysis (FEA) is an effective alternative tool to perform nonlinear analysis on the specimens, which can perform parametric studies by altering parameters in the FEA model precisely and effectively. With the major failure modes of steel beam column connection including weld fracture and beam flange local and lateral buckling, RBS show promising seismic performance to mitigate these failure modes and comply the strong column weak beam design concept to ensure the flexure strength of column is strong in column than the flexure strength of beam. As a result, excessive deformation of column which can lead to collapse of structure can be prevented.

\subsection{Conclusion}

Two exterior cantilever steel moment connections from the corresponding test program were modeled and studied. Two different type of geometry of RBS were investigated by using FEA from Strand7 software. Nonlinear hysteresis response of the beam tip load versus deflection and moment versus rotation response were generated and 
show correlation results compared to experimental results obtained from the test program. Plate element is suitable to model the RBS connection due to the smaller element numbers and less degree of freedoms generated, therefore shorter solution time required. Parametric studies of the RBS connection can be conducted with plate element FEA modeling to produce effective results which can be used to evaluate experimental results and do comparison studies. Yet plate element model predicted the specimen's moment strength deteriorates at a faster rate compared to the experimental results. While brick element model accurately demonstrates the strength deterioration rate of the specimen, the model over-estimates the ultimate strength of the connection. Therefore, tuning the material properties of yield and tensile strength definition for the FEA brick mesh modeling can produce finer results. Moreover, from comparison between RBS FEA result with full beam section FEA result, it showed improved of ductility for the connection by using RBS, and plastic hinge relocated in beam flange cut. On the other hand, nonlinear response results show that the geometry affects the hysteresis behavior of $\mathrm{RB}$, such that radius cut RBS exhibit greater seismic performance including better ductility and moment resistance than constant cut RBS.

Model frame with RBS was modeling in Strand7 and nonlinear time history FEA was performed. Results show that the deformation of the frame is no longer elastic, which is indicated in the roof displacement curve doesn't oscillate along the origin. Hence permanent plastic deformation occurs along the seismic ground motion simulation. Base shear versus displacement result was extracted from Strand7, and frame with RBS reveals greater ductility compared to frame with full beam section. In addition, incremental dynamic analysis by using the nonlinear transient dynamic solver in Strand7 was 
conducted to assess the behavior of the moment frames under increase of seismic loads. The maximum roof displacement was predicted for the structure is subjected to seismic loads that with different scaled ground motion. Consequently, the limit state of the structural deformation can be estimated with this analysis to effectively comply with steel moment beam column connection design.

\subsection{Future Work}

Brick element modeling in Strand7 of RBS connections and MRF were efficient but requires skillful modeling technique. In addition, brick element modeling is presumed to present higher accuracy of solutions for higher demand of complex geometry with details which can be modelled with contact elements in Strand7. Future research can be conducted to investigate the suitable finite element modeling method to produce design models with finer mesh with appropriate contact elements, which is subjected to bigger cyclic loading with magnitude over 6 in displacement load. Therefore, it can reveal how the connection details affects the nonlinear seismic response of the RBS. Nevertheless, the location of CJP welds and weld access holes are relatively difficult to inspect in a large model with big numbers of elements, consideration of grouping and defining numerous quantities of elements with well-suited material properties become critical to the attain the productive result. Furthermore, extended studies can be carried on with incremental dynamic analysis in Strand7 to examine the seismic response of RBS special moment resisting frames with multiple stories structure. Various of ground motions can be generated in the nonlinear transient dynamic solver to generate nonlinear analysis results that to predict capacity and the limit state of the structure. The result will be 
valuable for performance-based assessment and design for buildings in seismic zone, which can ensure buildings can withstand its functionality during earthquake event.

\section{References}

1. Afzalan, M. \& Ghassemieh, M. (2015). Finite Element Modeling of Failure in Steel Moment Connection Subjected to Ultra-low Cycle Fatigue Loading. The 2015 World Congress on Advances in Civil, Environmental and Material Research (ACEM15).

2. American Institute of Steel Construction. ANSI/AISC 341: Seismic provisions for Structural Steel Buildings, Chicago, Illinois, 2016

3. American Institute of Steel Construction. ANSI/AISC 358-16: Prequalified Connections for Special and Intermediate Steel Moment Frames for Seismic Applications, Chicago, Illinois, 2016

4. American Institute of Steel Construction. Steel Construction Manual, Chicago, Illinois, 2015

5. AISC (2003). "Prequalified Connections for Special and Intermediate Steel Moment

6. Frames for Seismic Applications (DRAFT)", American Institute of Steel Construction, 
Chicago, Illinois

7. Al-Shawwa, N., \& Lignos, D. (2013). Web-Based Interactive Tools for Performance-Based Earthquake Engineering. Retrieved April 15, 2018, from http://dimitrios-lignos.research.mcgill.ca/databases/steel/report.php?s_id=EngelhardtE9608-UTDB1

8. ASTM (2000). “Annual Book of ASTM Standards, 2000, section three, Metals Test Methods and Analytical Procedures”, volume 03.01, West Conshohocken, Pennsylvania

9. ASTM (2002). “Annual Book of ASTM Standards, 2002, section one, Iron and Steel Products", volume 01.04, West Conshohocken, Pennsylvania

10. ATC. (2014). Guidelines for Nonlinear Structural Analysis for Design of Buildings Part IIa - Steel Moment Frames. Applied Technology Council, Redwood City, CA

11. AWS (1995). "Specification for Carbon Steel Electrodes for Flux Cored Arc Welding”, ANSI/AWS A5.20-95, AWS, Miami, Florida

12. Brunesi, E., Nascimbene, R., Rassati, G. (2014). Response of partially-restrained bolted beam-to- column connections under cyclic loads. Journal of Constructional Steel Research. 97. pp 24-38. 
13. Chi, B. and Uang, C. (2002). "Cyclic Response and Design Recommendations of Reduced Beam Section Moment Connections with Deep Columns”, Journal of StructuralEngineering, ASCE, Vol. 128, Issue 4, pp. 464-473

14. Dardis, J. (2016). What's so special about steel special moment frames? Retrieved March 5, 2019, from https://www.aisc.org/globalassets/modern-steel/archives/2016/10/sw.pdf

15. Ebrahimi, A., Jamkhaneh, M. \& Amiri, M. (2018). "3D Finite-Element Analysis of Steel Moment Frames Including Long-Span Entrance by Strengthening Steel Cables and Diagonal Concentrically Braced Frames under Progressive Collapse”, Practice Periodical on Structural Design and Construction, 23(4). DOI:

10.1061/(ASCE)SC.1943-5576.0000388

16. Elkady, A., Lignos, D.G. (2017). "Full scale testing of Deep Wide Flange Steel Columns under Multi-Axis Cyclic loading: Loading Sequence, Boundary Effects and Out-of-plane Braced Force Demand", Journal of Structural Engineering, 14(2). DOI: 10.1061/(ASCE)ST.1943-541X.0001937.

17. Ebrahimi, A., Jamkhaneh, M. \& Amiri, M. (2018). "3D Finite-Element Analysis of Steel Moment Frames Including Long-Span Entrance by Strengthening Steel Cables and Diagonal Concentrically Braced Frames under Progressive Collapse”, Practice 
Periodical on Structural Design and Construction, 23(4). DOI:

10.1061/(ASCE)SC.1943-5576.0000388

18. El-Tawil, S. \& Deierlein, GG. (2001). "Nonlinear analyses of mixed steel-concrete moment frames. Part I- beam-column element formulation”, Journal of Structural Engineering, ASCE 127(6).

19. Elkady, A., Lignos, D.G. (2017). Full scale testing of Deep Wide Flange Steel Columns under Multi-Axis Cyclic loading: Loading Sequence, Boundary Effects and Out-of-plane Braced Force Demand. Journal of Structural Engineering, 14(2). DOI: 10.1061/(ASCE)ST.1943-541X.0001937.

20. Engelhardt, M., Winneberger, T., Zekany, A., Potyraj, T. (1998). "Experimental Investigation of Dog-bone Moment Connections," Engineering Journal, American Institute of Steel Construction, Vol. 35, pp. 128-139.

21. FEMA 350. (2000). Recommended seismic design criteria for new steel moment-frame buildings. Prepared by the SAC Joint Venture for the Federal Emergency Management Agency, Washington, DC.

22. Ferraioli, M., Lavino, A., \& Mandara, A. (2014). Behaviour Factor of code-design steel moment-resisting frames. International Journal of Steel Structures, 14(2), pp 243-254. 
23. "Pre-Northridge" Moment-Resisting Frame Buildings. (n.d.). Retrieved from https://forell.com/pre-northridge/

24. Foster, P., Abdelal, G., Lim, J. B. P., Hajsadeghi, M., \& McCrum, D. (2016). Finite element modelling of cyclic behaviour of cold-formed steel bolted moment-resisting connections. Journal of Construction in Developing Countries, 21(1), pp 167-180. DOI: 10.21315/jcdc2016.21.1.9.

25. International Code Council. International Building Code (IBC). Falls Church, Virginia, 2012

26. Jablonska-Krysiewicz, A. (2015). Finite Element Modeling of The Behavior of Steel End-Plate Connection. Journal of Civil Engineering, Environment and Architecture. pp $173-184$

27. Jia, Y. \& Yamada, S. (2018). "Loading Protocols for Steel Beam Tests in Seismic Performance Evaluation”, Integrating Science, Engineering \& Policy, Los Angeles, California

28. Jin, J.\& El-Tawil, S. (2004). "Seismic performance of steel frames with reduced beam section connections", Journal of Constructional Steel Research, Vol.61, pp.453471 
29. Jones, S., Fry, G., and Engelhardt, M. (2002). "Experimental Evaluation of Cyclically Loaded Reduced Beam Section Moment Connections". Journal of Structural Engineering, 128(4). DOI: 10.1061/(ASCE)0733-9445(2002)128:4(441)

30. Kulkarni, S., and Vesmawala, G. (2014). "Study of steel moment connection with and without reduced beam section", Case Studies in Structural Engineering 1, pp. 26-31

31. Malley, J. (1998) "Steel Project: Summary of Phase 1 testing investigation results", Engineering Structures, Vol. 20, Issues 4-6, pp. 300-309.

32. Moradi, S. \& Alam, S. (2015). Finite-Element Simulation of Posttensioned Steel Connections with Bolted Angles under Cyclic Loading. American Society of Civil Engineers: Journal of Structural Engineering, 142(1).

33. Naughton,D.,Tsavdaridis, K.\&Maraveas,C. (2018). "Pushover Analysis of Steel Seismic Resistant Frames with Reduced Web Section and Reduced Beam Section Connections", Frontier in Build Environment, Vol.3.

34. SAC Joint Venture. (2000). Recommended Specifications and Quality Assurance Guidelines for Steel Moment-Frame Construction for Seismic Applications.

35. Seif,M., Mcallister,T., Main,J \&Luecke, W.(2016). “Finite Element Modeling of Steel Moment Connections with Fracture for Structural Fire Analyses", Engineering Journal, pp.48-59. 
36. Stojadinovic, B., Goel, S., Lee, K., Margarian, A., \& Choi, J. (2000). "Parametric Tests on Unreinforced Steel Moment Connections”, Journal of Structural Engineering, 126(1), 40-49.

37. Strand7 Webnotes (2010), Strand7 Pty Ltd, Sydney, Australia

38. Strand7. (2010) Using Strand7, Edition 3, Strand7 Pty Ltd, Sydney, Australia.

39. Strand7. (2015) Strand7 Reference Manual, Release 2.4, Strand7 Pty Ltd, Sydney, Australia

40. Zhang, X., \& Ricles, J. M. (2004). Seismic Performance of Wide Flange Beam to Deep -column Moment Connections, ProQuest Dissertations and Thesis.

41. Zhang, X., \& Ricles, J. M. (2006). "Seismic Behavior of Reduced Beam Section Moment Connections to Deep Columns" Journal of Structural Engineering, ASCE, Vol. 132, Issue 3, pp. 358-367 


\section{Appendix}

Table A.1 Procedure to Calculate Node Mass for RBS MRF

\begin{tabular}{|c|c|c|c|c|c|c|}
\hline & & & & $\begin{array}{l}\text { duced } \\
\text { Beam } \\
\text { ection }\end{array}$ & $\begin{array}{r}\text { Regular Beam } \\
\text { Section }\end{array}$ & \\
\hline Modulus Elasticity & $\mathrm{E}$ & & & 29000 & 29000 & ksi \\
\hline Moment of Inertia & Ic & & & 6600 & 6600 & in^4 \\
\hline Column Height & $\mathrm{h}$ & & & 136 & 136 & in \\
\hline $\begin{array}{l}\text { beam to column } \\
\text { ratio }=(\mathrm{EIb} / \mathrm{L}) /(2 \mathrm{EIc} / \mathrm{h} \\
\text { ) }\end{array}$ & $\mathrm{r}=(\mathrm{EIb} / \mathrm{L}) /(2$ & $\mathrm{EIc} / \mathrm{h})$ & 0.2262 & $\begin{array}{r}24202 \\
3 \\
\end{array}$ & 0.331905758 & \\
\hline Reduce Flange Width & $\mathrm{c}$ (Flange $\mathrm{Cu}$ & & & 2.375 & 2.375 & in \\
\hline Bay Length & $\mathrm{L}$ & & & 80.62 & 280.62 & in \\
\hline $\begin{array}{l}\text { The moment of inertia } \\
\text { calculated as }\end{array}$ & at the center $\mathrm{c}$ & f the $r$ & duced $b$ & $\frac{5.505}{\text { eam sec }}$ & tion is & $\mathrm{tt}$ \\
\hline $\mathrm{Ib}$ & & & 040.00 & in^4 & & \\
\hline $\begin{array}{l}\text { I RBS }=\text { Ix- } 4 *\left(1 / 12 *{ }^{*} *^{t}\right. \\
\left.\text { tf) } / 2+\mathrm{tf} / 2)^{\wedge} 2\right)\end{array}$ & $3+c * t f^{*}((d-$ & & 5162.07 & in`$^{\wedge}$ & & \\
\hline & & $\begin{array}{l}\text { Redu } \\
\text { Beam } \\
\text { Secti }\end{array}$ & & & $\begin{array}{l}\text { Regular } \\
\text { Beam } \\
\text { Section }\end{array}$ & \\
\hline $\begin{array}{l}\text { The lateral stiffness ke } \\
\left.E I c / h^{\wedge} 3\right)\left(12^{*} p+1\right) /\left(12^{*}\right.\end{array}$ & $\begin{array}{l}(24 \\
p+4) \\
\end{array}$ & 1010 & 285806 & kip/in & $\begin{array}{r}1139.87419 \\
1 \\
\end{array}$ & kip/in \\
\hline & & 1010 & 285.806 & $1 \mathrm{~b} /$ in & $\begin{array}{r}1139874.19 \\
1\end{array}$ & lb/in \\
\hline & & 1771 & 33410.4 & $\mathrm{~N} / \mathrm{m}$ & $\begin{array}{r}199854141 . \\
9\end{array}$ & $\mathrm{~N} / \mathrm{m}$ \\
\hline $1 \mathrm{~m}=$ & & & 39.4 & in & 39.4 & in \\
\hline $1 \mathrm{LB}=$ & & & 4.45 & $\mathrm{~N}$ & 4.45 & $\mathrm{~N}$ \\
\hline $1 \mathrm{~kg}=$ & & & 2.2 & LB & 2.2 & LB \\
\hline Period T & & & 0.75 & $\mathrm{~s}$ & 0.75 & $\mathrm{~s}$ \\
\hline & & & $\begin{array}{l}\text { luced } \\
\text { tion }\end{array}$ & & $\begin{array}{l}\text { Regular } \\
\text { Beam } \\
\text { Section } \\
\end{array}$ & \\
\hline Translational Mass Al & ng Y-Axis & 2526 & 09.372 & $\mathrm{~kg}$ & $\begin{array}{r}2850469.46 \\
2 \\
\end{array}$ & $\mathrm{~kg}$ \\
\hline & & 5558 & 00.618 & LB & $\begin{array}{r}6271032.81 \\
7\end{array}$ & LB \\
\hline Each Node Mass & & 2779 & 50.309 & LB & $\begin{array}{r}3135516.40 \\
9\end{array}$ & LB \\
\hline
\end{tabular}

